\title{
DOE/CH/03000--T28
}

\section{MICRODOSIMETRIC INVESTIGATIONS AT THE FAST NEUTRON THERAPY FACILITY AT FERMILAB}

RECEIVED

By

Katja Maria Langen
JAII 26 998

$08 \mathrm{TI}$

A DiSSERTATION SUBMITTED IN PARTIAL FULFILLMENT OF THE

- REQUIREMENTS FOR THE DEGREE OF

Doctor of PhILOSOPhY

(Department of Medical Physics)

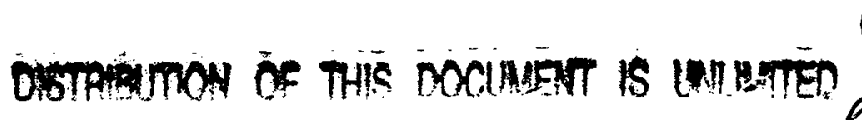

at the

UNIVERSITY OF WISCONSIN - MADISON 


\section{DISCLAMMER}

This report was prepared as an account of work sponsored by an agency of the United States Government. Neither the United States Government nor any agency thereof, nor any of their employees, makes any warranty, express or implied, or assumes any legal liability or responsibility for the accuracy, completeness, or usefulness of any information, apparatus, product, or process disclosed, or represents that its use would not infringe privately owned rights. Reference herein to any specific commercial product, process, or service by trade name, trademark, manufacturer, or otherwise does not necessarily constitute or imply its endorsement, recommendation, or favoring by the United States Government or any agency thereof. The views and opinions of authors expressed herein do not necessarily state or reflect those of the United States Government or any agency thereof. 


\section{DISCLAIMER}

Portions of this document may be illegible electronic image products. Images are produced from the best available original document. 


\title{
MICRODOSIMETRIC INVESTIGATIONS AT THE FAST NEUTRON THERAPY FACILITY AT FERMILAB
}

\author{
By \\ Katja Maria Langen
}

\begin{abstract}
A DisSERTATION SUBMITTED IN PARTIAL FULFILLMENT OF THE REQUIREMENTS FOR THE DEGREE OF
\end{abstract}

DOCTOR OF PHILOSOPHY

(Department of Medical Physics)

\author{
at the \\ UNIVERSITY OF WISCONSIN - MADISON \\ 1997
}


M ICRO D O SIM ETRIC INVESTIGATION S AT

THE FAST NEUTRON

THER A P Y FACILITYA T FER M ILA B

\title{
Katja Maria Langen
}

Under the supervision of Professor Paul M. DeLuca, Jr.

At the University of Wisconsin - Madison

\begin{abstract}
Microdosimetry was used to investigate three issues at the neutron therapy facility (NTF) at Fermilab.

Firstly, the conversion factor from absorbed dose in A-150 tissue equivalent plastic to absorbed dose in ICRU tissue was determined. For this, the effective neutron kerma factor ratios, i.e. oxygen to A-150 tissue equivalent plastic and carbon to A-150 tissue equivalent plastic, were measured in the neutron beam. An A-150 tissue equivalent plastic to ICRU tissue absorbed dose conversion factor of $0.92 \pm 0.04$ was determined.

Secondly, variations in the radiobiological effectiveness (RBE) in the beam were mapped by determining variations in two related quantities, $e^{*}$ and $R$, with field size and depth in tissue. Maximal variation in $e^{*}$ and $R$ of $9 \%$ and $15 \%$ respectively were determined.

Lastly, the feasibility of utilizing the boron neutron capture reaction on boron-10 to selectively enhance the tumor dose in the NTF beam was investigated. In the unmodified beam, a negligible enhancement for a $50 \mathrm{ppm}$ boron loading was measured. To boost the boron dose enhancement to $3 \%$ it was necessary to change the primary proton energy from $66 \mathrm{MeV}$ to $37 \mathrm{MeV}$ and to filter the beam by 90 $\mathrm{mm}$ of tungsten.
\end{abstract}


The feasibility of measuring microdosimetric spectra in the NTF beam was proven and its usefulness demonstrated. 


\section{Acknowledgements}

I would like to thank my advisor, Professor Paul M. DeLuca, Jr., for the guidance, support and opportunities that I have received from him during the last five years. To work for him was very inspiring and a lot of fun. Many thanks also to Drs. Alan Ross and Tim Bohm for their help with the Monte Carlo calculations and with various UNIX problems.

During my time at Fermilab I have received help from numerous people. I would like to thank Dr. Arlene Lennox, head of the neutron therapy facility (NTF), for her continuing support of this project. Thanks to Tom Kroc and the rest of the NTF staff for helping me with many details. I'd like to thank Larry Allen for tuning the NTF beam so many times, particularly for tuning the beam twice last New Year's eve. I thank Lester Wahl for helping with many equipment problems. I am grateful to Chuck Schmidt for thinking of an alternative method to reduce the NTF dose rate which has saved me uncountable hours. I also appreciate the friendliness and helpfulness of all the main control room crews.

On a personal note, I thank my parents for their encouragement and for complaining so very little about my absence. My sister-in-law, Birgit, and my brother, Georg, I thank for helping me in so many ways.

I appreciate the financial support of this project through Fermilab's Beam Division's Ph.D. program. 


\section{List of Figures}

1 Accelerator setup at Fermilab (not to scale). . . . . . . . 8

2 The Neutron Therapy Facility at Fermilab (not to scale). . . . . . 10

3 The target and pre-collimator setup after Rosenberg et al. [43] (not to scale $\ldots \ldots \ldots \ldots \ldots \ldots \ldots \ldots$

4 A microdosimetric proportional counter. . . . . . . . . 16

5 The $\epsilon_{\text {sat }}$ function versus $\epsilon \ldots \ldots \ldots \ldots \ldots$

6 Biological weighting function, $\mathrm{r}(\epsilon)$ empirically determined by Pihet et al. [38]. . . . . . . . . . . . . . . . . 2 25

7 Setup of electronic signal processing equipment. . . . . . . . . . 29

8 Typical proportional counter setup in the treatment room. . . . . 30

9 The linac beam structure. . . . . . . . . . . . . . . . . 33

10 The modified beam structure, using Kroc's method [23]. . . . . . 33

11 Calculated ICRU tissue to A-150 plastic kerma factor ratio. Kerma factors were taken from Chadwick et al. [11] . . . . . . . . . . 37

12 Calculated neutron fluence spectrum for NTF [45]. . . . . . . 38

13 Carbon microdosimetry data. . . . . . . . . . . . . 40

14 Zirconium microdosimetry data. . . . . . . . . . . . . . . . 41

15 Zirconium oxide microdosimetry data . . . . . . . . . .442

16 "Oxygen" response microdosimetry data. . . . . . . . . . . . . 44

17 Microdosimetric spectra measured at a field size of $50 \mathrm{~mm} \times 50 \mathrm{~mm}$. 51

18 Microdosimetric spectra measured at a field size of $100 \mathrm{~mm} \times 100 \mathrm{~mm}$. 52

19 Microdosimetric spectra measured at a field size of $200 \mathrm{~mm} \times 200 \mathrm{~mm} .53$

20 Microdosimetric spectra measured at a depth of $50 \mathrm{~mm} . \ldots . .54$ 
21 Microdosimetric spectra measured at a depth of $100 \mathrm{~mm} . \ldots . .55$

22 Microdosimetric spectra measured at a depth of $150 \mathrm{~mm} \ldots \ldots$. . . 56

23 Spectra measured at $150 \mathrm{~mm}$ depth in a $50 \mathrm{~mm} \times 50 \mathrm{~mm}$ field and $50 \mathrm{~mm}$ depth in a $200 \mathrm{~mm} \times 200 \mathrm{~mm}$ field. . . . . . . . 57

24 Geometry used for Monte Carlo calculations. . . . . . . . . . . 64

25 Side view of counter setup in the treatment room for the open field BNC measurements. . . . . . . . . . . . . 66

26 Microdosimetric spectra measured in the unmodified beam. . . . . 67

27 Results of MCNP calculations on the effect of tungsten filtration of the NTF beam. . . . . . . . . . . . . . . . 68

28 Side view of counter setup in the treatment room for the tungsten filtered beam BNC measurements. . . . . . . . . . . . . . 69

29 Microdosimetric spectra measured in the tungsten filtered beam. . . 70

30 Side view of counter setup in the treatment room for the steel collimated BNC measurements. . . . . . . . . . . . . . . .71

31 Microdosimetric spectra measured in the steel block collimated field. 72

32 Microdosimetric spectra measured in the steel block collimated and tungsten filtered field. . . . . . . . . . . . . 73

33 Microdosimetric spectra measured in the $37 \mathrm{MeV}$ and tungsten filtered beam. . . . . . . . . . . . . . . . . 74

34 A-150 microdosimetric spectra measured in the modified and unmodified beam. . . . . . . . . . . . . . . 75 


\section{List of Tables}

1 Several fast neutron therapy facilities and some of their characteristics. 6

2 The diameter, density, mass and cross section of the tissue cell and counter cavity. ......................... 18

3 Conversion factors between $y, Y, D_{g}$ and $\epsilon$, for a $2 \mu m$ gas filling. . 20

4 Elemental composition (in \% weight) of ICRU muscle tissue and A-150 tissue equivalent plastic $[35,53] \ldots \ldots$. . . . . . . . 35

5 Intermediate results for the ICRU tissue to A-150 kerma factor ratio determination. .................... 41

$6 \epsilon^{*}$ and $R$ values relative to those obtained at a field size of $100 \mathrm{~mm} \times$ $100 \mathrm{~mm}$ and depth of $100 \mathrm{~mm}$................ 50 


\section{Contents}

Abstract

Acknowledgements $\quad$ iii

1 Introduction 1

2 Neutron Therapy 5

2.1 Development ..................... 5

2.2 Rationale ........................ 7

2.3 Neutron Therapy Facility (NTF) at Fermilab . . . . . . . . 7

3 Microdosimetry 11

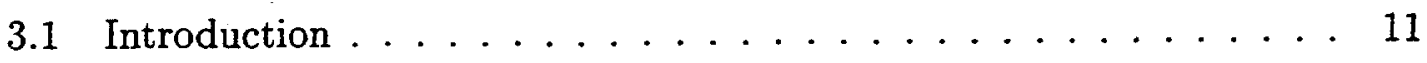

3.2 Neutron Interactions ................. 11

3.3 Absorbed Dose Determination . . . . . . . . . . . . 12

3.4 Instrumentation . . . . . . . . . . . . . 15

3.5 Volume Simulation ................... 17

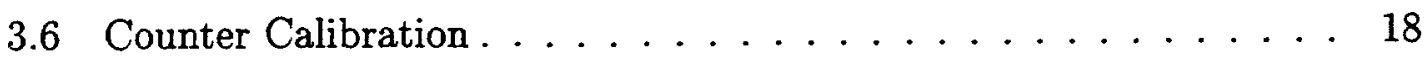

3.7 Data Analysis . . . . . . . . . . . . . . 20

3.8 Qualitative Information . . . . . . . . . . . . 23

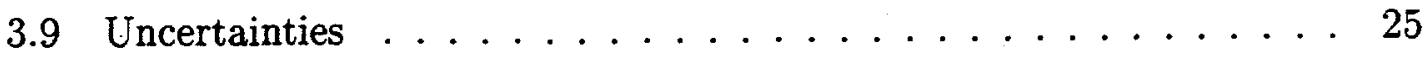

4 Experimental Methods $\quad 28$

4.1 Data Acquisition ...................... 28

4.2 Dose Rate Reductions . . . . . . . . . . . . . 31

5 Relative Carbon and Oxygen Kerma Factor Ratios 34

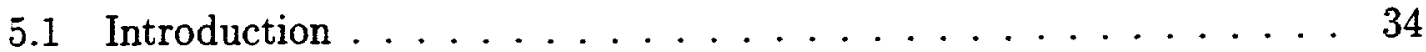

5.2 Correction of A-150 Reading . . . . . . . . . . 35 


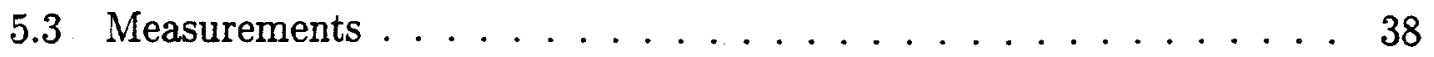

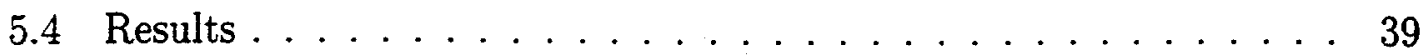

5.5 Discussion ........................ 45

6 Beam Characterization $\quad 46$

6.1 Introduction . . . . . . . . . . . . . . 46

$6.2 \mathrm{RBE}$ variations with depth in tissue . . . . . . . . . . . 48

6.3 RBE variations with field size . . . . . . . . . . . . 49

6.4 Measurements . . . . . . . . . . . . . . . . 49

6.5 Results ...................... 49

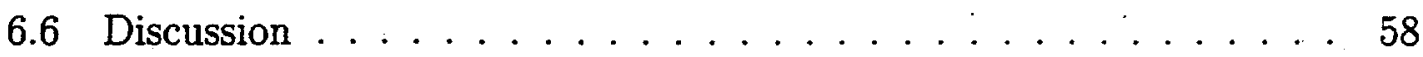

7 Boron Neutron Capture $\quad 60$

7.1 Introduction . . . . . . . . . . . . . 60

7.2 Clinical rationale ....................... 61

$7.3 \mathrm{BNC}$ in fast neutron beams .................. 62

7.4 Beam Modifications . . . . . . . . . . . . . . . . 62

7.5 Measurements ........................ 64

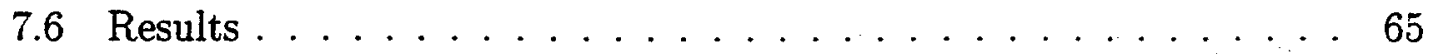

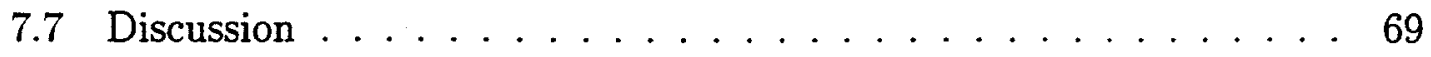

8 Conclusions $\quad 76$

$\begin{array}{ll}\text { Bibliography } & 78\end{array}$ 


\section{Chapter 1}

\section{Introduction}

The neutron therapy facility (NTF) at the Fermi National Accelerator Laboratory has been treating cancer patients since 1976. During more than twenty years of operation over 2300 patients have been treated and a wealth of clinical experience has been accumulated. Therefore the clinical effects of the neutron beam are well known and characterized.

Several important physical characteristics of the beam can be determined with ion chamber measurements. For example, central axis depth dose and off-axis dose ratios were quantified using an A-150 ion chamber [43]. There are, however, some physical characteristics that were thus far not directly determined in the NTF beam. These include the A-150 tissue equivalent plastic to ICRU tissue absorbed dose ratio. A calculation of this factor requires large amounts of data that are not completely available. Furthermore, qualitative variations of the neutron beam with irradiation conditions were investigated only sparsely in the NTF beam [19]. Microdosimetry can be used to investigate several issues, including the above, in a neutron beam, since it provides quantitative as well as qualitative information on the absorbed dose deposited in a neutron beam.

Microdosimetric measurements are acquired in a pulse mode and they require a sufficiently low dose rate to minimize pulse pile-up. The beam structure at NTF is such that it results in a very low duty cycle and the instantaneous dose rate within the beam pulse is consequently very high. The instantaneous dose rate needs to be reduced drastically to allow the measurement of microdosimetric data. This fact has thus far prohibited the utilization of microdosimetry in the NTF beam. 
The neutron therapy facility is part of Fermilab where a chain of accelerators provides a high energy proton beam primarily for research purposes. NTF uses protons from the linear accelerator (linac) when they are not needed for injection into a downstream booster synchrotron. The linac beam structure is dictated by the booster operation and a modification of it is not practical.

This thesis was initiated when a technique was developed to reduce the instantaneous dose rate sufficiently by modifying the intensity of beam pulses designated for NTF. Unfortunately this technique also further reduces the duty cycle of the beam, necessitating long data acquisition times. During the course of this project another technique was developed where the original duty cycle is maintained while the instantaneous dose rate is reduced to a level that allows microdosimetric meaurements to be taken. This latter technique was used to acquire the microdosimetric data presented in this thesis. These measurements are the first microdosimetric measurements taken in the NTF beam.

Employing microdosimetric techniques, several characteristics of the neutron therapy beam were investigated and a feasibility study on the utilization of boron neutron capture in the NTF beam was performed.

One investigation focused upon the conversion factor that relates absorbed dose in ICRU (International Commission on Radiation Units and Measurements) tissue to absorbed dose in A-150 plastic. The former is the most important quantity in radiation therapy while the latter is routinely measured for calibration purposes in neutron beams. A calculation of this factor is possible but requires kerma factor ratio values for all relevant neutron energies as well as knowledge of the neutron energy spectrum. Kerma factor ratios are only sparsely available for neutron energies above $30 \mathrm{MeV}$. In a recent study, Monte Carlo calculations were used to calculate the energy spectra for various facilities including $\mathrm{NTF}$ [45]. In this thesis microdosimetry was used to directly measure the quantities needed to determine the absorbed dose in ICRU tissue to absorbed dose in A-150 plastic conversion 
factor.

In a second investigation we used the qualitative information provided by microdosimetric measurements to map changes in the radiobiological effectiveness (RBE) of the beam. Traditionally, the RBE of a beam is determined by irradiation of cell cultures in both the beam of interest and in a reference beam. These experiments are very time consuming. Alternatively, microdosimetry offers the possibility of performing physical measurements that can be related to the biological effectiveness of the beam. The RBE, for a given biological endpoint, of a neutron beam depends on the neutron energy spectrum which changes slightly with beam parameters such as field size and depth in tissue. The influence of both these parameters on the radiation quality were investigated in the Fermilab beam.

Last, a feasibility study on the use of boron neutron capture therapy (BNCT) in the NTF beam was performed. The ultimate goal in radiation therapy is to deliver a lethal absorbed dose to tumor tissue, but for each treatment modality the absorbed tumor dose is eventually limited by the absorbed dose given to surrounding normal tissue in the course of treatment. The aim of treatment planning is to deliver a maximal amount of absorbed dose to the tumor while minimizing the absorbed dose to normal tissue. An approach to conform dose to tumor tissue is to selectively sensitize the tumor tissue thereby improving the tumor to normal tissue dose ratio. Boron neutron capture therapy is based on this method. When tumor tissue is selectively loaded with a boron compound and exposed to thermal neutrons the neutron capture reaction on boron 10 , which results in two short range densely ionizing particles, can be used to selectively enhance the tumor dose.

We explored the possibility of using this method to enhance the tumor dose in Fermilab's fast neutron therapy beam. The dose enhancement can be calculated if the boron concentration in the tumor as well as in normal tissue and the thermal neutron fluence at the tumor location are known. Instead, the boron dose enhancement was measured directly using a tissue equivalent microdosimetric counter with 
a boron-loaded wall and a regular tissue equivalent microdosimetric counter.

It is a further aim to increase the boron dose boost in the neutron beam. Besides increasing the boron concentration one can try to increase the thermal neutron fluence at the tumor location to get a further enhancement. The latter method necessitates a change in the neutron energy spectrum. This can be achieved by either beam filtration or by an alteration of the primary neutron energy spectrum. Both options were investigated in the NTF beam. Microdosimetric measurements, to determine the boron dose enhancement, were performed in the modified beams. This thesis is divided into eight chapters. The following two chapter provide background information on neutron therapy and microdosimetry. The fourth chapter describes the experimental setup used. Following are three chapters that each deal with one of three investigations. A conclusion chapter is last. 


\section{Chapter 2}

\section{Neutron Therapy}

\subsection{Development}

When the first patient was treated with neutrons at Lawrence Berkeley Laboratory in 1938 little was known about the radiobiology of neutrons, except that for the same exposure neutron irradiation resulted in a greater biological effect than $\mathrm{x}$-ray irradiation. First, single large dose treatments were applied until a new cyclotron became available for clinical work, permitting fractionated therapy [55]. A total of 250 patients were treated until 1943 when the project was halted by World War. II. In 1948, after studying the clinical results, Stone concluded that the neutron therapy treatments resulted in such severe late side effects that they should not be continued [54].

Interest in neutron therapy rose again at the Hammersmith Hospital in London some twenty years later, after it became apparent that the oxygen enhancement ratio (OER) for neutrons was reduced compared to x-rays and many tumors, particularly larger tumors, were thought to contain a hypoxic cell fraction. This group re-investigated Stone's clinical results and attributed the severe late effects in part to the increased radiobiological effectiveness (RBE) of neutrons with decrease in the dose per fraction [13]. After extensive radiobiological experiments neutron therapy treatments were started in the late sixties at Hammersmith. Initial clinical results were promising and this triggered the installation of several neutron therapy facilities in the US and Europe.

Subsequent clinical trials could not reproduce the initial Hammersmith results. The majority of these new facilities were built around existing accelerators which 


\begin{tabular}{|c|c|c|}
\hline Facility & Neutron Reaction & Comments \\
\hline \hline Seattle, WA & $\mathrm{p}(50) \mathrm{Be}$ & Isocentric Gantry \\
& $\mathrm{Multileaf}$ Collimator \\
Batavia, IL & $\mathrm{p}(66) \mathrm{Be}$ & Horizontal Beamline \\
Detroit, MI & $\mathrm{d}(48.5) \mathrm{Be}$ & Isocentric Gantry \\
& & Multirod Collimator \\
Seoul, Korea & $\mathrm{d}(51) \mathrm{Be}$ & Isocentric Gantry \\
Faure, South Africa & $\mathrm{p}(66) \mathrm{Be}$ & Isocentric Gantry \\
& $\mathrm{Variable}$ Collimator \\
Louvain-La-Neuve, Belgium & $\mathrm{p}(65) \mathrm{Be}$ & Vertical Beamline \\
& & Multileaf Collimator \\
\hline
\end{tabular}

Table 1: Several fast neutron therapy facilities and some of their characteristics.

necessitated compromises in the facility design. Most importantly; the neutron energy spectra of these facilities were too low to achieve adequate depth dose penetration. However, the emergence of conflicting clinical results cannot be explained based on this ground since the Hammersmith facility also used a low energy neutron beam. The Hammersmith results are exceptional and must be due to other factors.

In the mid-seventies, facilities came on-line that used high energy ( $\geq 50 \mathrm{MeV}$ ) charged particles to generate neutron beams. These facilities are referred to as fast neutron therapy facilities. At these facilities the depth dose penetration is comparable to 6-8MV photon beams. The three currently operating US facilities all use fast neutron beams. Table 1 lists several facilities and their technical attributes.

It should be noted that none of the listed facilities is truly identical to another facility. Even facilities that use charged particles of the same type and energy to generate neutron fields are not necessarily identical in target thickness or collimation. The neutron energy spectra and consequently the radiobiological effectiveness 
of the beam can be influenced by all these parameters and unless facilities are identical in all aspects clinical results from different facilities have to be compared with caution [59].

\subsection{Rationale}

The radiobiological rationale of neutron therapy has evolved since treatments commenced again at Hammersmith. The importance of the reduced oxygen enhancement ratio (OER) was questioned when it was discovered that some hypoxic cells reoxygenate during fractionated treatment [56]. Reoxygenation is slower in slow growing tumors which indicates an enhanced sensitivity of slowly growing tumors to neutron therapy [58]. In addition, the radiosensitivity variation over a cell cycle that exists for photon irradiation is less pronounced for neutron irradiation. Cells that have a long cell cycle, i.e., they are slow growing and have a longer G1 phase in which they are relatively insensitive to photon irradiation, are less protected if irradiated with neutrons [16].

The clinical value of neutron therapy is still under investigation. Its advantage in the treatment of locally advanced inoperable salivary gland tumors is generally accepted [15]. Studies point towards an advantage of neutron therapy in the treatment of locally advanced prostate cancer [24, 47]. Next to these, there are other tumors that can be treated with neutron therapy. Wambersie estimates that $10-15 \%$ of all referred radiotherapy patients can benefit from neutron therapy $[58,60]$. This number also stresses the need for proper patient selection.

\subsection{Neutron Therapy Facility (NTF) at Fermilab}

Unlike other facilities, NTF utilizes protons from a linear accelerator in a parasitic mode. The proton linac is part of a series of accelerators that eventually accelerate protons up to $900 \mathrm{GeV}$. Its primary function is to accelerate $750 \mathrm{keV}$ protons to 


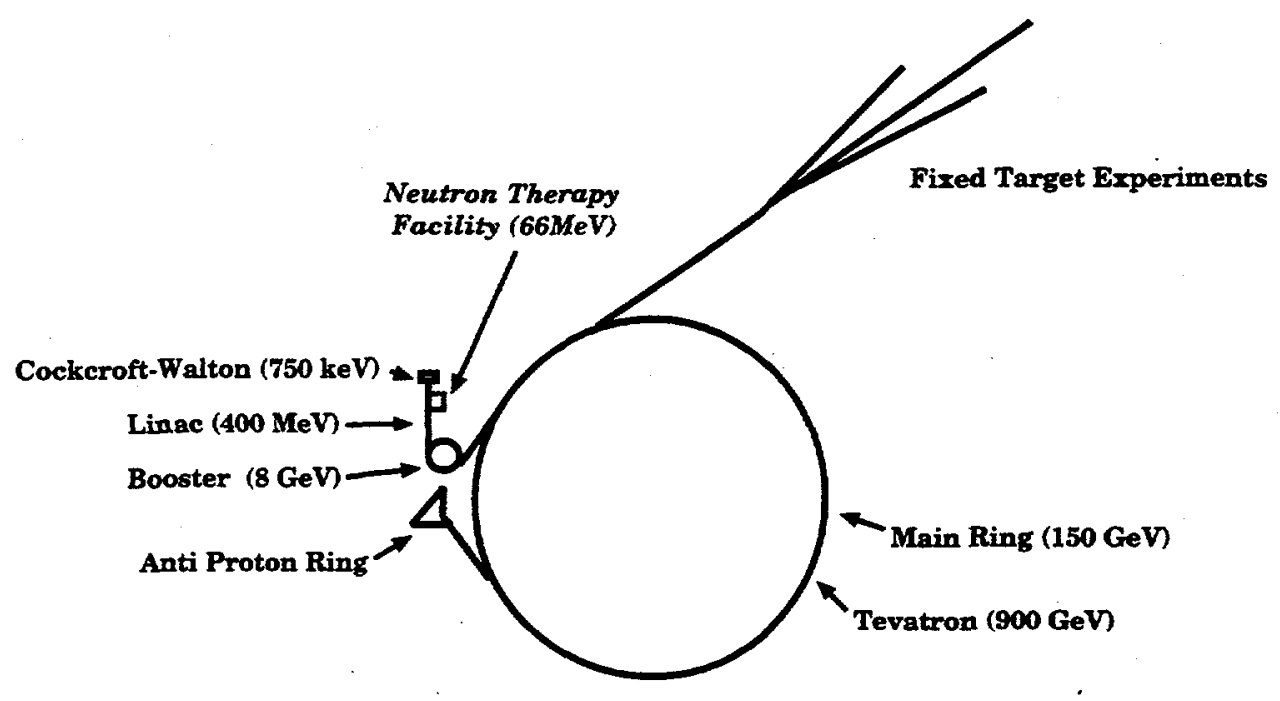

Figure 1: Accelerator setup at Fermilab (not to scale).

an energy of $400 \mathrm{MeV}$ and then inject them into a booster synchrotron. After being further accelerated in the booster synchrotron the protons enter the main accelerator ring before being finally injected into the tevatron. Since the booster requires injection only for a fraction of time, protons are available for neutron therapy the rest of the time. Figure 1 shows the layout of the acceleration chain at Fermilab.

Since NTF was built at an existing and operating proton linac its design had to be compatible with the accelerator operation. The linac is four feet below ground level and for economical reasons it was decided to treat patients at the linac level and to use one of the three existing freight elevators to move patients down to that level. Freight elevators are located at the beginning, middle and end of the linac building. At the time when NTF was designed, protons at these locations had an energy of $750 \mathrm{keV}, 99 \mathrm{MeV}$ and $200 \mathrm{MeV}$ respectively. Based on these energies the freight elevator that is located at the middle of the linac building was chosen to be used for neutron therapy [26]. At the time, the linac itself was 
divided into nine linac tanks and beam can only be extracted between two linac tanks. The chosen elevator is located between linac tank four and five. Protons exiting tank four have an energy of $99 \mathrm{MeV}$. However, the space between the linac tanks is such that with a conventional magnet only protons up to $65-70 \mathrm{MeV}$ could be cleanly extracted [28]. Protons leaving linac tank three have an energy of 66 $\mathrm{MeV}$ and in order to use these protons, linac tank four is turned off while beam pulses designated for NTF are passing through. After drifting through tank four protons for NTF are extracted using a magnet that bends protons by 58 degrees. The first dipole is followed by a second dipole to complete a 90 degree bending of the beam. The beam exits through a shielding wall before impinging onto a beryllium target. Figure 2 -illustrates the beam-line design of the facility. In the early nineties the proton linac was upgraded to accelerate protons to $400 \mathrm{MeV}$ but this did not interfere with NTF operations since all upgrades were implemented downstream of NTF.

A patient is set up at ground level where a laser array is used to simulate the beam isocenter. For treatment the elevator is lowered to the linac level where an equivalent laser array is used to position the patient. After the treatment is completed the elevator is raised again to the ground level.

For the Fermilab facility a $22.1 \mathrm{~mm}$ thick beryllium target was chosen in which $66 \mathrm{MeV}$ protons lose $49 \mathrm{MeV}$. This configuration is abbreviated as $\mathrm{p}(66) \mathrm{Be}(49)$. The residual proton energy is absorbed in a $0.5 \mathrm{~mm}$ gold foil [43]. The collimation system consists of a primary collimator that is followed by a secondary assembly in which interchangeable collimators for different field sizes can be placed. Figure 3 illustrates the target and collimator design. The total length of the collimation system is $1.09 \mathrm{~m}$ with a target to isocenter distance of $1.90 \mathrm{~m}$ [43]. Due to the fixed horizontal beam line the patient sits in a chair or stands on a platform at isocenter. Typical dose rates at isocenter are $40 \mathrm{rad} / \mathrm{min}$ or $0.4 \mathrm{~Gy} / \mathrm{min}$ at $100 \mathrm{~mm}$ depth in tissue equivalent liquid. 


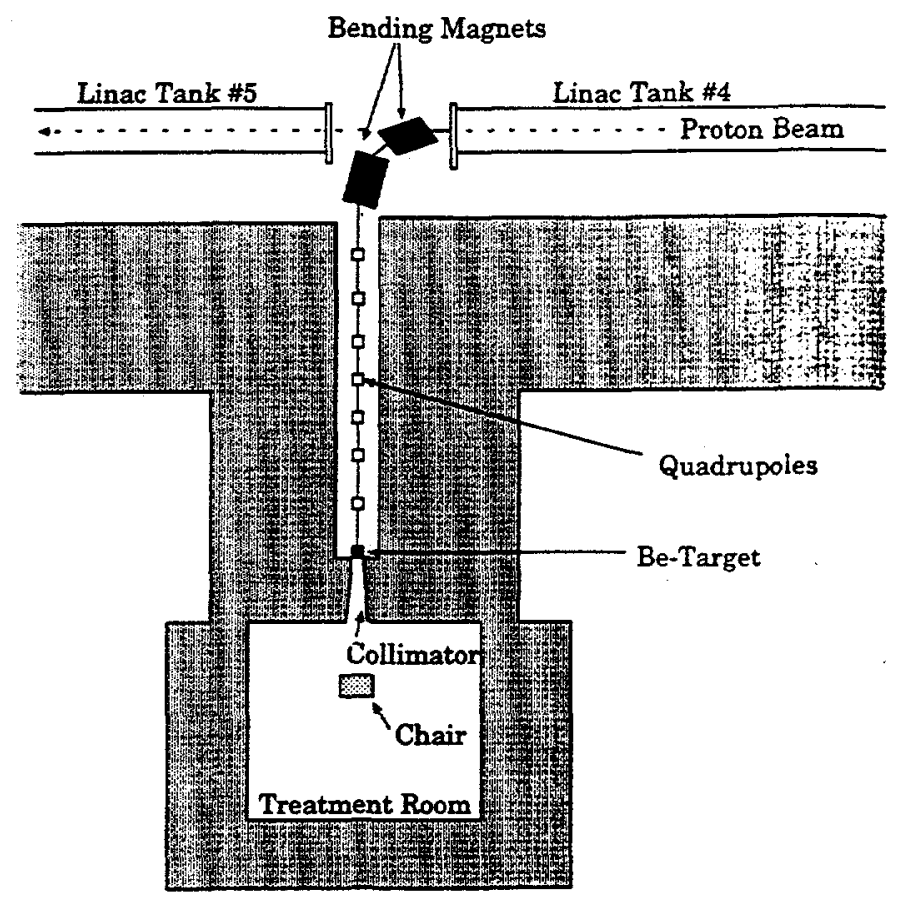

Figure 2: The Neutron Therapy Facility at Fermilab (not to scale).

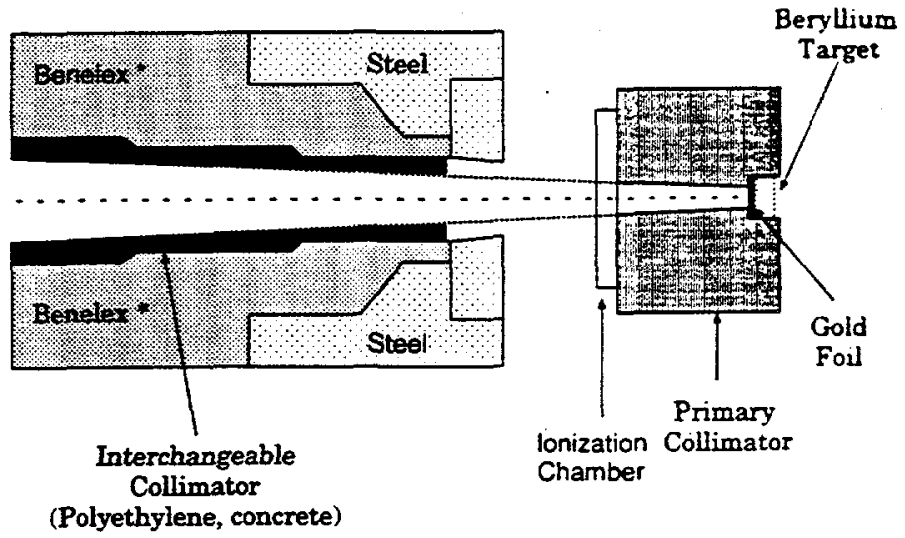

*Trade name: American Masonite
Corporation, Chicago, IL, USA

Figure 3: The target and pre-collimator setup after Rosenberg et al. [43] (not to scale). 


\section{Chapter 3}

\section{Microdosimetry}

\subsection{Introduction}

Microdosimetry is the study of energy deposition events on a micrometer scale. A micrometer size cell volume is simulated by filling a centimeter size cavity with tissue equivalent gas to a low pressure such that charged particles crossing the gas cavity lose an equal amount of energy in the gas and in a micrometer size tissue volume. The amount of energy lost by a charged particle per unit path length, i.e., the stopping power, depends on the particle type and energy. The frequency distribution of energy deposition events collected in the counter is therefore a direct consequence of the charged particle spectrum crossing the counter cavity which is itself intimately connected to the primary radiation spectrum. In neutron fields, the shape of a microdosimetric spectrum is correlated with the radiation quality of the primary field [30]. Microdosimetry therefore provides quantitative as well as qualitative information on the energy deposited in a material in a radiation field. It can be applied in any radiation field but it is especially powerful in neutron fields due to the large variety of secondary particles generated in these fields.

\subsection{Neutron Interactions}

Neutrons deposit their energy indirectly by interaction with the nuclei of the irradiated material. As a result of these nuclear interactions, secondary particles (protons, alphas, heavy recoils) are generated. These secondary charged particles then deposit their energy in the absorbing material. Type and probability of a 
particular reaction occurring, depends on the neutron energy and target nuclei.

Neutron interactions with target nuclei can be classified into several categories.

- elastic scattering $(n, n)$

- inelastic scattering $\left(n, n^{\prime}\right)$

- nonelastic scattering ( $n$,charged particle)

- capture ( $n, \gamma$ or $n$, charged particle)

In tissue, elastic scattering on hydrogen nuclei is the dominant reaction in terms of neutron energy transfer up to neutron energies of about $30 \mathrm{MeV}$. Due to the similarity of neutron and proton masses the maximum energy can be transfered in a neutron-proton collision. Nonelastic reactions on carbon and oxygen contribute significantly to the neutron energy transfer in tissue and tissue-like materials at neutron energies above $30 \mathrm{MeV}$ [11]. The capture of thermal neutrons by hydrogen nuclei is the main contributer to the gamma contamination in a therapeutic neutron beam [5].

\subsection{Absorbed Dose Determination}

A fundamental quantity in dosimetry is the energy imparted $\epsilon$ in a volume by ionizing particles [36].

$$
\epsilon=R_{\text {in }}-R_{\text {out }}+\sum Q[J]
$$

$R_{\text {in }}$ is the sum of energies of all charged and uncharged particles entering the volume, $R_{\text {out }}$ is the sum of energies of all charged and uncharged particles leaving the volume and $\sum Q$ is the sum of all rest mass changes that occur in the volume. A related quantity, the specific energy (imparted) $z$, is defined as 


$$
z=\frac{\epsilon}{m}\left[J k g^{-1}\right]
$$

where $\epsilon$ is the energy imparted to a mass $\mathrm{m}$ [36].

The absorbed dose $D$ is defined to be

$$
D=\frac{d \bar{e}}{d m}\left[J k g^{-1}\right] \text { or }[G y]
$$

where $d \bar{e}$ is the mean energy imparted to the mass $d m$ [36]. The absorbed dose $D$ is related to the mean specific energy,

$$
\bar{z}=\int_{0}^{\infty} z f(z) d z\left[J k g^{-1}\right]
$$

by

$$
D=\lim _{m \rightarrow 0} \bar{z}
$$

where $f(z)$ is the frequency distribution of $z$. The absorbed dose is defined as the energy imparted in an infinitesimal volume at a point and $\mathrm{dm}$ is the mass of this volume. The absorbed dose is therefore the expectation value of the specific energy $z$. A measurement of absorbed dose is really a measurement of the absorbed dose averaged over a volume with mass $\mathrm{dm}$. The imparted energy $\epsilon$ and specific energy $z$ are both stochastic quantities whereas as the mean specific energy $\bar{z}$ and absorbed dose $\mathrm{D}$ are non-stochastic quantities [36].

Kerma, $\mathrm{K}$, is the amount of kinetic energy $d E_{t r}$ given to charged particles by indirectly ionizing radiation per unit mass.

$$
K=\frac{d E_{t r}}{d m}\left[J k g^{-1}\right] \text { or }[G y]
$$

The absorbed dose refers to the absorption of energy in matter whereas kerma refers to the transfer of energy to charged particles in matter. Under charged 
particle equilibrium (CPE) conditions, kerma is numerically equal to absorbed dose which can be measured by a variety of methods.

$$
\text { Kerma } \stackrel{\mathrm{CPE}}{=} \text { Dose }[G y]
$$

CPE conditions exist when the secondary charged particle energy spectrum entering a volume is equal to the charged particle energy spectrum leaving the volume, i.e. the energy given to charged particles in a volume is equal to the energy deposited in the volume by charged particles.

Using cavity theory formalism [1] the absorbed dose measured in a material $\mathrm{g}$ can be related to the absorbed dose in a material $\mathrm{w}$. The absorbed dose $\mathrm{D}$ in material $w$ is equal to

$$
D_{w}=\Phi \times\left(\frac{d E}{d l \rho}\right)_{c, w}[G y]
$$

where $\Phi$ is the charged particle fluence and $\left(\frac{d E}{d l \rho}\right)_{c, w}$ the mass collision stopping power in material $w$. Near the interface between material $w$ and $g$, the charged particle fluence can assumed to be constant. The ratio of absorbed dose in the materials is then

$$
\frac{D_{w}}{D_{g}}=\frac{\left(\frac{d E}{d l \rho}\right)_{c, w}}{\left(\frac{d E}{d l \rho}\right)_{c, g}} .
$$

Using $\bar{S} / \rho$ to denote the average mass collision stopping power of a charged particle spectra, equation 3.9 can be rewritten for a spectrum of charged particles. The ratio of dose in material $\mathrm{w}$ to dose in material $\mathrm{g}$ is

$$
\frac{D_{w}}{D_{g}}=\frac{(\bar{S} / \rho)_{w}}{(\bar{S} / \rho)_{g}}=\left(\frac{\bar{S}}{\rho}\right)_{g}^{w} .
$$

This is known as the Bragg-Gray relation [1]. This principle can be extended to a gas filled cavity and in a material. A constant charged particle fluence in the cavity 
and throughout the material, is the underlying condition and has to be fulfilled. To achieve this certain conditions must be true.

1. The charged particle fluence is not perturbed in the gas cavity, i.e., the size of the gas cavity is small compared to the secondary particle range.

2. The dose to the gas is only due to particles crossing the gas cavity (Only for dissimilar gas and wall materials).

The second Bragg-Gray condition is difficult to fulfill for counters exposed to a neutron fluence. This introduces some uncertainty to the dose determination.

\subsection{Instrumentation}

Microdosimetric spectra were measured with low pressure proportional counters (manufactured by Far West Technology, Goleta, California, USA), also called Rossi-counters. The design was first developed by Rossi et al. [46] in the fifties. Figure 4 is a diagram of a typical counter. Counters are spherical with a $12.7 \mathrm{~mm}$ inner diameter. Walls can be made of several materials of interest and are typically $1.27 \mathrm{~mm}$ thick. Most commonly counters are used to measure absorbed dose in A-150 tissue equivalent (TE) plastic and are therefore made with TE walls. Counters were filled with isobutane based tissue equivalent gas (elemental composition in percentage weight is $\mathrm{H}: 10.3, \mathrm{C}: 59.2, \mathrm{~N}: 3.5, \mathrm{O}: 26.9$ [49]) to a low pressure (66 Torr) to simulate a $2 \mu \mathrm{m}$ diameter sphere of unit density tissue. The counter wall is grounded and the anode is biased to $+600 V_{D C}$. A helical grid which is biased to $20 \%$ of the anode voltage is centered around the anode to correct for field inhomogeneities at the anode entrance and exit to the cavity. An internal alpha particle source can be used for calibration purposes. The whole assembly is encapsulated in a vacuum-tight aluminum housing. 


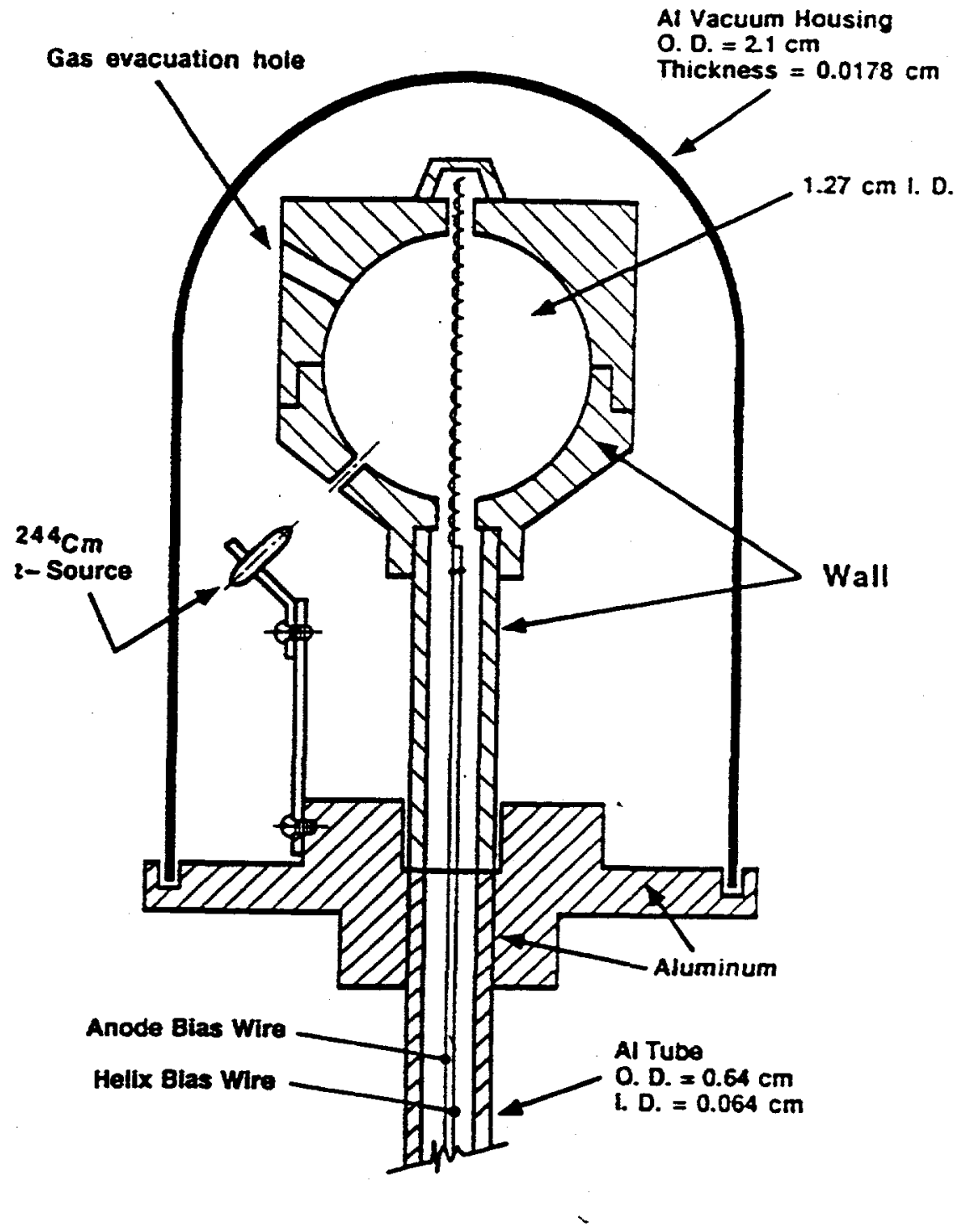

Figure 4: A microdosimetric proportional counter. 
Radiation interacts with the wall material and secondary charged particles enter the cavity where they produce ionization in the counting gas. Electrons travel towards the anode where gas multiplication occurs. A pulse with amplitude proportional to the number of electrons produced in the counting gas is induced at the anode. The counter is operated in pulse mode and the pulse height distribution is collected and stored.

\subsection{Volume Simulation}

Microdosimetric counters are filled with low pressure gas to simulate a $2 \mu \mathrm{m}$ tissue volume. This means that a charged particle loses the same amount of energy $\bar{\epsilon}$ when it crosses the gas cavity diameter and when it goes through $2 \mu \mathrm{m}$ of unit density tissue, i.e.

$$
\bar{\epsilon}=d_{g} \times\left(\frac{S}{\rho}\right)_{g} \times \rho_{g}=d_{t} \times\left(\frac{S}{\rho}\right)_{t} \times \rho_{t}[\mathrm{MeV}]
$$

where $\mathrm{d}$ is the sphere diameter, $\left(\frac{S}{\rho}\right)$ is the collision mass stopping power, $\rho$ is the density and subscripts $g$ and $t$ indicate gas and tissue. For a particle of the same type and energy passing through the same material, equation 3.11 reduces to

$$
d_{g} \times \rho_{g}=d_{t} \times \rho_{t}
$$

or

$$
\rho_{g}=\frac{d_{t}}{d_{g}} \times \rho_{t}\left[\mathrm{~g} / \mathrm{cm}^{3}\right]
$$

This allows us to define a factor $k_{t}^{g}$.

$$
k_{t}^{g}=\frac{d_{g}}{d_{t}}
$$




\begin{tabular}{|l|c|r|}
\hline Quantity & Tissue cell & Counter cavity \\
\hline \hline Diameter $[\mathrm{m}]$ & $2 \times 10^{-6}$ & $12.7 \times 10^{-3}$ \\
Density $\left[\mathrm{kg} / \mathrm{m}^{3}\right]$ & $1 \times 10^{3}$ & $157.5 \times 10^{-3}$ \\
Mass $[\mathrm{kg}]$ & $4.19 \times 10^{-15}$ & $169 \times 10^{-9}$ \\
Cross section $\left[\mathrm{m}^{2}\right]$ & $31.4 \times 10^{-13}$ & $1.27 \times 10^{-4}$ \\
\hline
\end{tabular}

Table 2: The diameter, density, mass and cross section of the tissue cell and counter cavity.

For a spherical gas cavity and cell, the gas mass in the counter is $\left(k_{t}^{g}\right)^{2}$ times the cell mass. An energy deposition event due to a particle crossing the counter diameter results in an absorbed dose in the counter that is $\left(k_{t}^{g}\right)^{2}$ times smaller than the absorbed dose in a cell due to an identical event since the imparted energy is the same in both cases. On the other hand, the counter cross section is also $\left(k_{t}^{g}\right)^{2}$ times larger than the cell cross section and the therefore $\left(k_{t}^{g}\right)^{2}$ times more particles cross the counter cavity. For the same fluence, the absorbed dose in the cell and counter is therefore identical. However, there are $\left(k_{t}^{g}\right)^{2}$ times more events in the counter per unit of absorbed dose [37]. Table 2 lists the diameters, densities, masses and cross sections for the counter cavity and tissue cell. The event size distribution as measured in the counter does by no means simulate the event size distribution of a single cell, the counter rather simulates the event size distribution experienced by a collection of $\left(k_{t}^{g}\right)^{2}$ cells.

\subsection{Counter Calibration}

The pulse height for any particle that traverses the cavity is proportional to the energy it deposits in the cavity, and since the mass of the gas is constant, the pulse height is also proportional to the absorbed dose in the gas due to that particle. However, the pulse height is not proportional to the stopping power of a particle since its path length through the cavity is unknown. This raises the question of 
pulse height calibration.

According to ICRU Report 36 [37], microdosimetric spectra can be expressed in terms of lineal energy $y$,

$$
y=\frac{\epsilon}{\bar{\ell}}[k e V / \mu m]
$$

where $\epsilon$ is the energy imparted in the counter and $\bar{\ell}$ is the mean chord length in the cell volume. For a sphere the mean chord length $\bar{\ell}$ is $2 / 3 d, d$ being the sphere diameter. The use of lineal energy implies that the measured pulse height is proportional to $\frac{d E}{d x}$, or linear energy transfer of the particle. Since this is not so, the concept of lineal energy is somewhat misleading. Only for a particle whose path length is equal to $2 / 3 d$, is $y$ numerically equal to its stopping power. However, particles going through $2 / 3 d$ can not be identified in the pulse height spectrum.

Alternatively, spectra can be expressed in terms of $Y$,

$$
Y=\frac{\epsilon}{m_{g}} \times \frac{V_{g}}{d_{g}}\left[\mathrm{MeVcm}^{2} / g\right] .
$$

where $m_{g}$ is the gas mass, $V_{g}$ and $d_{g}$ are counter volume and diameter. The utilization of $Y$ has similar drawbacks as that of lineal energy $y$. The display of microdosimetric spectra in terms of $Y$ has the only advantage that, if a particle crosses the diameter of the cavity, $Y$ is numerically equal to its stopping power. Certain points, namely the proton and alpha edge, in a microdosimetric spectrum can be associated with particles that cross the diameter of the counter.

It is furthermore possible to display the spectrum in terms of absorbed gas dose $D_{g}$.

$$
D_{g}=\frac{\epsilon}{m_{g}} \cdot[\mathrm{MeV} / \mathrm{g}]
$$

The pulse height is proportional to the absorbed dose in the gas and an event can be clearly associated with the absorbed dose deposited by it. However, the absorbed 


\begin{tabular}{|c|l|l|}
\hline Unit & Definition & Conversion \\
\hline \hline$y$ & $y=\frac{\epsilon}{2 / 3 d}[\mathrm{keV} / \mu \mathrm{m}]$ & $y=752 \times \epsilon[\mathrm{MeV}]$ \\
\hline$Y$ & $Y=\frac{\epsilon}{m_{g}} \times \frac{V_{g}}{d_{g}}\left[\mathrm{MeV} \mathrm{cm}{ }^{2} / g\right]$ & $Y=5263 \times \epsilon[\mathrm{MeV}]$ \\
\hline$D_{g}$ & $D_{g}=\frac{\epsilon}{m_{g}}[\mathrm{MeV} / \mathrm{g}]$ & $D_{g}=5882 \times \epsilon[\mathrm{MeV}]$ \\
\hline
\end{tabular}

Table 3: Conversion factors between $y, Y, D_{g}$ and $\epsilon$, for a $2 \mu m$ gas filling.

dose in the gas is $\left(k_{t}^{g}\right)^{2}$ times smaller than the absorbed dose in a cell deposited by an identical particle. Since a microdosimetric counter is used to simulate a cell, it is advantageous to display the pulse height spectrum in a quantity that is identical in the counter and cell.

For this work all spectra were calibrated in terms of imparted energy $\epsilon$. The pulse height is directly proportional to the energy imparted by a particle. In addition, the imparted energy due to a particle is identical in the counter and in a cell.

The conversion between $y, Y, D_{g}$ and $\epsilon$ is linear and table 3 list the appropriate conversion factors.

\subsection{Data Analysis}

In an ion chamber the imparted energy $\epsilon$ is

$$
\epsilon=Q \times \frac{\bar{W}}{e} \times 10^{-6}[\mathrm{MeV}]
$$

where $\mathrm{Q}$ is the number of elemental charges collected and $\bar{W}$ is the average energy required in $\mathrm{eV}$ produce an ion pair. For a proportional counter equation 3.18 has to be expanded to include a gas multiplication factor $\mathrm{g}$.

$$
\epsilon=\frac{Q}{g} \times \frac{\bar{W}}{e} \times 10^{-6}[\mathrm{MeV}]
$$


Since gas multiplication only occurs in close proximity to the anode it is independent of the location of the initial ionization event. A constant gas multiplication factor can be assumed.

The $\frac{\bar{W}}{e}$ value depends on the charged particle type and energy. Since it is not possible to clearly distinguish between different particle types and energy, a constant $\frac{\bar{W}}{e}$ value is used. This introduces some uncertainty in the measurement which will be further discussed in section 3.9. The necessity of the counter calibration arises from the fact that the gas multiplication factor $\mathrm{g}$ is undetermined.

A Cm-244 calibration source is built into the counter assembly which can be used for counter calibration and performance testing. Cm-244 emits two $\alpha$ particles, a $5.806-\mathrm{MeV}$ particle with a $76.7 \%$ probability and a $5.764-\mathrm{MeV}$ particle with $23.3 \%$ emission probability [25], which results in an average $\alpha$ energy of $\bar{E}_{\alpha}=5.8 \mathrm{MeV}$. The alphas are collimated to cross the counter diameter. Using the known mass collision stopping power $S / \rho$ for the alpha particle in TE-butane gas, the counter diameter $d_{g}$ as the path length, and the gas density $\rho_{g}$, the deposited energy $\epsilon$ in the counter gas is

$$
\epsilon=\frac{S}{\rho} \times \cdot d_{g} \times \rho_{g}[\mathrm{MeV}] .
$$

In this way, the alpha source can be used to determine the gas gain $g$ and to calibrate the pulse heights in terms of imparted energy $\epsilon$.

This procedure assumes knowledge of the $\alpha$ particle energy. The $\alpha$ sources used in these counters have a gold coating to seal the source. Alpha particles lose energy in this gold layer and its thickness therefore determines the energy of the $\alpha$ particles that enter the gas cavity. Substantial differences in $\alpha$ particle energies due to variations in the gold layer thickness have been reported $[10,57]$.

To reduce uncertainties associated with a $\alpha$-source calibration, proton edge calibration was used to calibrate spectra. This method is based on the fact that, 
for each particle type there is a maximum amount of energy that can deposit in the counter. Firstly, theses particles have a maximal stopping power while they traverse the counter cavity and secondly, they must travel along the longest path length in the counter, i.e. go through the counter diameter. For a $2 \mu \mathrm{m}$ equivalent filling pressure with tissue equivalent gas, protons and alpha particles, that have a maximal stopping power can deposit up to $186 \mathrm{keV}$ and $505 \mathrm{keV}$, respectively, in the counter $[9,57]$. The frequency distribution in pulse height can then be calibrated in terms of imparted energy $\epsilon$.

The frequency distribution in imparted energy can be converted to dose distribution in imparted energy by

$$
D(\epsilon)=\epsilon \times f(\epsilon) \times \frac{1}{m_{g}} \times 1.602 \times 10^{-13}[G y]
$$

where $D(\epsilon)$ is the dose deposited in the gas by events of event size $\epsilon$ to $\epsilon+\Delta \epsilon, f(\epsilon)$ is the number of events of imparted energy $\epsilon+\Delta \epsilon$, and $m_{g}$ is the gas mass in $\mathrm{kg}$. A microdosimetric spectra can be represented as $\epsilon D(\epsilon)$ vs. $\log \epsilon$. This representation conserves the equal area equal dose relationship of $D(\epsilon)$ vs. $\epsilon$. The total absorbed gas dose can be determined by the summation of $D(\epsilon)$ over $\epsilon$.

Using equation 3.10 the absorbed gas dose $D_{g}$ can be used to determine the absorbed wall dose $D_{w}$.

$$
\frac{D_{w}}{D_{g}}=S_{g}^{w}=r_{m, g}
$$

For a single particle of known type and energy, that crosses the counter cavity, the $r_{m, g}$ value is equal to the ratio of its stopping power in the wall and gas material. However, in a neutron field several types of particles with wide ranges of energies are generated. Newhauser et al. [32] used Monte Carlo codes to calculate charged particle emission spectra and mass stopping powers in a variety of materials and for a variety of neutron energies. Their publication supplies $r_{m, g}$ values for several wall 
and gas material combinations. Using these values, wall doses were determined.

\subsection{Qualitative Information}

To predict the biological effect of neutron irradiation two parameters are needed. Firstly, the absorbed dose in tissue needs to be known, but secondly, the radiobiological effectiveness (RBE) of the beam, i.e., its relative effectiveness as compared to $250-\mathrm{keV}$ gamma irradiation [16], needs to be known. The RBE of a neutron beam directly influences the clinical response to the beam and an increase or decrease in RBE can be equated with an increase or decrease in absorbed dose.

Through radiobiological experiments where cells were irradiated with charged particles of different types and energies, it was determined that RBE is a strong function of the linear energy transfer coefficient (LET), i.e., the energy lost by a charged particle per unit path length [3]. The RBE vs. LET function rises with LET but then peaks at a LET value of about $100 \mathrm{keV} / \mu \mathrm{m}$. With further increasing LET the RBE decreases. This is known as the saturation effect. An explanation for this effect is the deposition of excess energy in a target volume. If more than the necessary energy to cause a biological effect is deposited in a target no additional advantage is expected. The excess energy is wasted and the effectiveness is decreased.

This effect has been incorporated in the calculation of saturation corrected mean lineal energy $y^{*}[21]$.

$$
y^{*}=\int y_{\text {sat }}(y) \times d(y) d y
$$

Here $y_{\text {sat }}(y)$ is a biological weighting function that can be used to weight the microdosimetric spectra and $d(y)$ is the dose corresponding to events with lineal energy $\mathrm{y}$. The term $y^{*}$ is a weighted average that can be used to indicate the radiation quality. Using the conversion factor from table $3, y_{\text {sat }}$ was converted into 


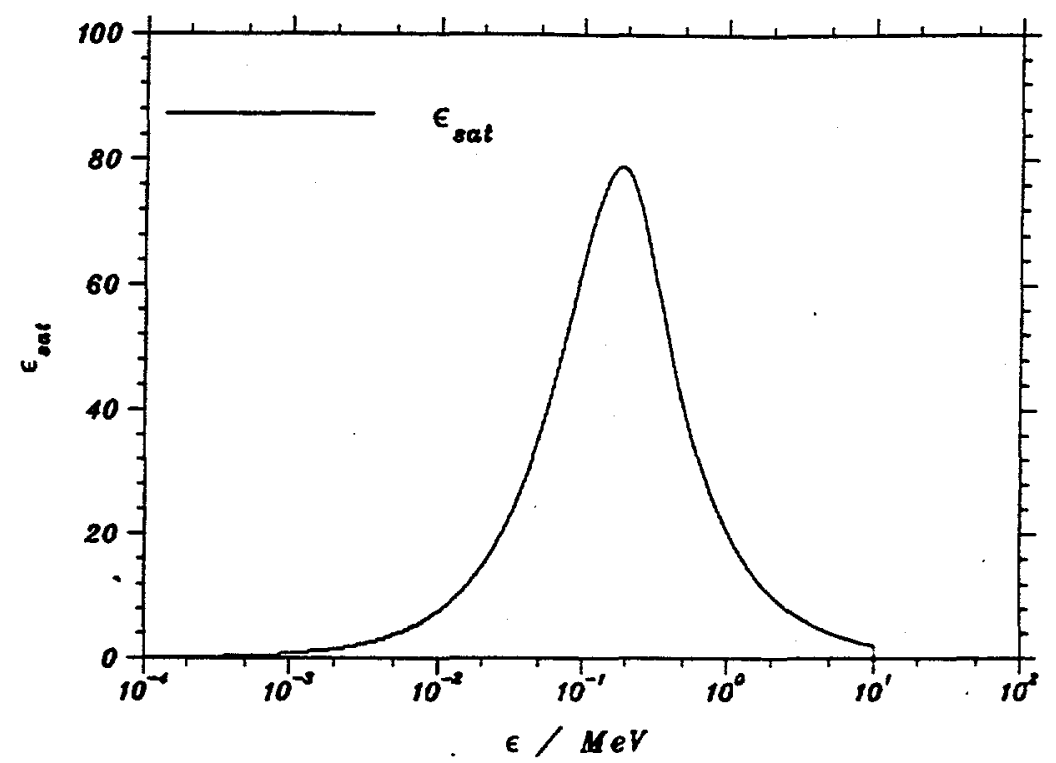

Figure 5: The $\epsilon_{\text {sat }}$ function versus $\epsilon$.

a $\epsilon_{\text {sat }}$ function that allows the calculation of $\epsilon^{*}$.

$$
\epsilon^{*}=\int \epsilon_{\text {sat }}(\epsilon) \times d(\epsilon) d \epsilon
$$

According to equation 3.24 , tissue equivalent microdosimetric spectra were multiplied with the $\epsilon_{\text {sat }}$ function on a bin by bin basis. Changes in the microdosimetric spectrum shape result in changes in $\epsilon^{*}$ and relative changes in $\epsilon^{*}$ were used to indicate RBE changes. Figure 5 shows the $\epsilon_{\text {sat }}$ function versus $\epsilon$.

Alternatively, Pihet et al. $[38,41]$ determined an empirical weighting function, $r(y)$, by combining tissue equivalent microdosimetric spectra measured at several neutron therapy facilities with radiobiological data obtained in the same beams under identical irradiation conditions.

$$
R=\int r(y) \times d(y) d y
$$

The $r(y)$ function was determined for early effects in mice (jejunum crypt cell 


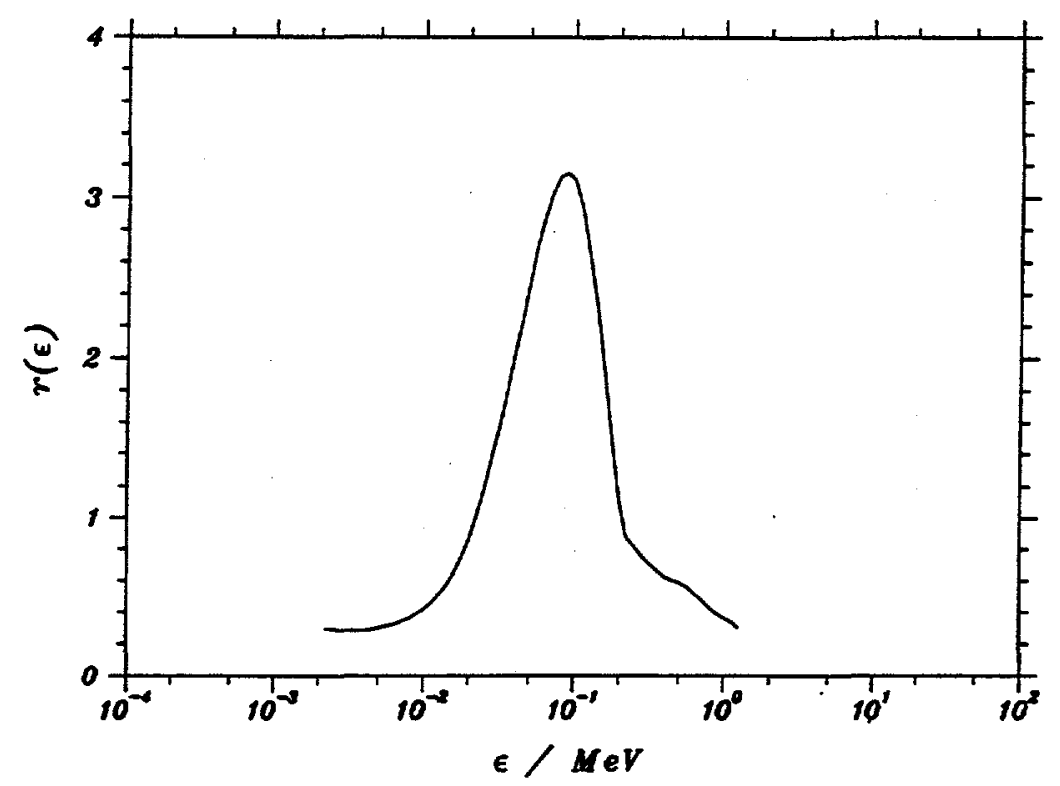

Figure 6: Biological weighting function, $r(\epsilon)$ empirically determined by Pihet et al. [38].

survival, skin reaction). The $r(y)$ function was converted for this work to a $r(\epsilon)$ function which is shown in Figure 6. A R-value was then determined according to the following equation.

$$
R=\int r(\epsilon) \times d(\epsilon) d \epsilon
$$

\subsection{Uncertainties}

There are several sources of uncertainties in microdosimetric measurements. Counting statistics, in addition to errors associated with the calibration technique, the use of a constant $\frac{\bar{W}}{e}$ value and the failure to fulfill the second Bragg-Gray cavity theory contribute to the total uncertainty. When wall kerma is determined, the uncertainty of $r_{m, g}$ values has to be included in an error analysis. The influence of 
these uncertainties on a particular measurement depends on the individual experimental conditions but for a single A-150 dose measurement the uncertainty in our data is typically $7 \%$. This is due to a combination of the following uncertainties.

Counting statistics: Since the probability that an event is of event size $\epsilon$ is small, Poisson statistics can be applied to estimate this error. If $N$ events have an event size $\epsilon$, their statistical error is $\sqrt{N}$. Waker et al. [57] estimated the total error by calculating microdosimetric quantities from two spectra, one in which each bin contained $N+\sqrt{N}$ events and another one with each bin containing $N-\sqrt{N}$ events. However, since the uncertainty that is introduced by one bin is independent from that of another bin, the total error in our data due to counting statistics was estimated by summing the uncertainties from all bins in quadrature. Typically, measured A-150 spectra have an uncertainty of 3-4 \% in absorbed dose due to counting statistics.

Calibration: For this work all tissue equivalent spectra were calibrated using the proton edge method. Carbon spectra were calibrated using the alpha edge method. Uncertainties in the proton edge method are estimated to contribute a $3 \%$ uncertainty to the determination of absorbed gas dose [39].

Constant $\frac{\bar{W}}{e}$ value: The $\frac{\bar{W}}{e}$ value determines the ratio of imparted energy $\epsilon$ to collected charge $Q$ (Equation 3.18) and is therefore of importance. The $\frac{\bar{W}}{e}$ value depends on the particle type and energy. Since neither the particle type nor energy can be uniquely identified for events in a microdosimetric spectrum a constant $\frac{\bar{W}}{e}$ value, i.e. that of the calibration events, is assumed. By assuming a constant $\frac{\bar{W}}{e}$ value for all events a $3 \%$ uncertainty in the gas dose determination is introduced [18, 39].

Second Bragg-Gray cavity theory condition: The violation of the second Bragg-Gray cavity condition for counters that differ in wall and gas composition has to be addressed. In this work three such counters $\left(\mathrm{C}, \mathrm{Zr}, \mathrm{ZrO}_{2}\right)$ were used. 
The error introduced in the $\mathrm{Zr}$ and $\mathrm{ZrO}_{2}$ counters is not of practical importance since it largely cancels out when the $\mathrm{Zr}$ spectrum is subtracted from the $\mathrm{ZrO}_{2}$ spectrum. At $15 \mathrm{MeV}$ neutron energy Bühler et al. [9] estimated that about 5.5 $\%$ of the gas dose in a TE gas filled carbon counter is due to "Starters", i.e. particles that are generated by neutron interactions in the gas. Since the TE kerma is about twice as large as the carbon kerma, this effect adds about a $3 \%$ uncertainty. At $50 \mathrm{MeV}$ neutron energy Newhauser [33] estimated an uncertainty of less than $1 \%$ due to this effect. The average neutron energy at the Fermilab neutron therapy facility is about $25 \mathrm{MeV}$, therefore a conservative estimate of the induced uncertainty in the dose determination is $2 \%$.

$r_{m, g}$ values: Newhauser et al. [32] estimated that their calculated $r_{m, g}$ values have a $4 \%$ uncertainty. 


\section{Chapter 4}

\section{Experimental Methods}

\subsection{Data Acquisition}

In each data acquisition run, one or two proportional counters were irradiated in the therapeutic neutron beam. Pulses from each counter were amplified in a charge sensitive pre-amplifier. Three NIM bin standard linear amplifiers with relative gain settings of $\times 10, \times 100$ and $\times 1000$ further amplified the pulses. The amplified pulses were then digitized by three Wilkinson-type analog to digital converters (ADC) into 1024 channels each. The ADC outputs were connected to a parallel I/O card in a 386 IBM compatible computer. Figure 7 illustrates the setup of the electronic signal processing equipment. Data were then sorted and displayed by a data acquisition program. For further analysis the data were transferred to a Unix computer.

The high voltage supplies were directly connected to the counters. The preamplifiers were connected via short $(<100 \mathrm{~mm})$ cables to the counters. To minimize noise pickup in the data transmission cables, it was necessary to amplify the pre-amp pulses in the treatment room before feeding them via $15 \mathrm{~m}$ long cables to the medical control room (MCR) where the ADCs were located. All additional data acquisition equipment was located in the MCR. Each ADC was gated with a beam envelope gate to minimize noise contamination.

Data were calibrated by determining the proton edge pulse height. This pulse height was assigned to event size $\epsilon=186 \mathrm{keV}$. The relative gain of other pulse heights was determined using a precision pulser. A pulser spectrum was taken before and after each run to determine drifts in the electronic equipment. Pulser 


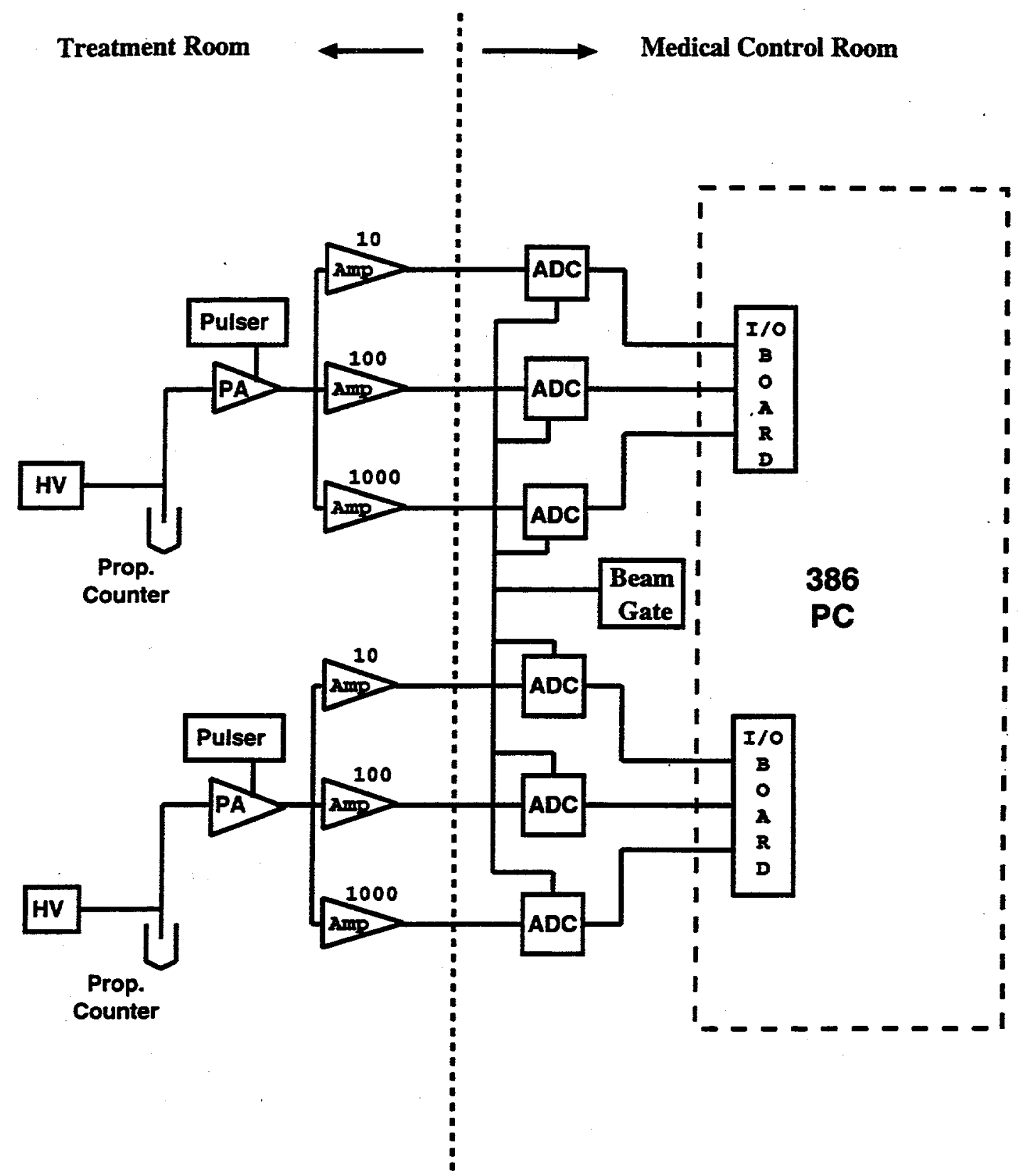

Figure 7: Setup of electronic signal processing equipment. 


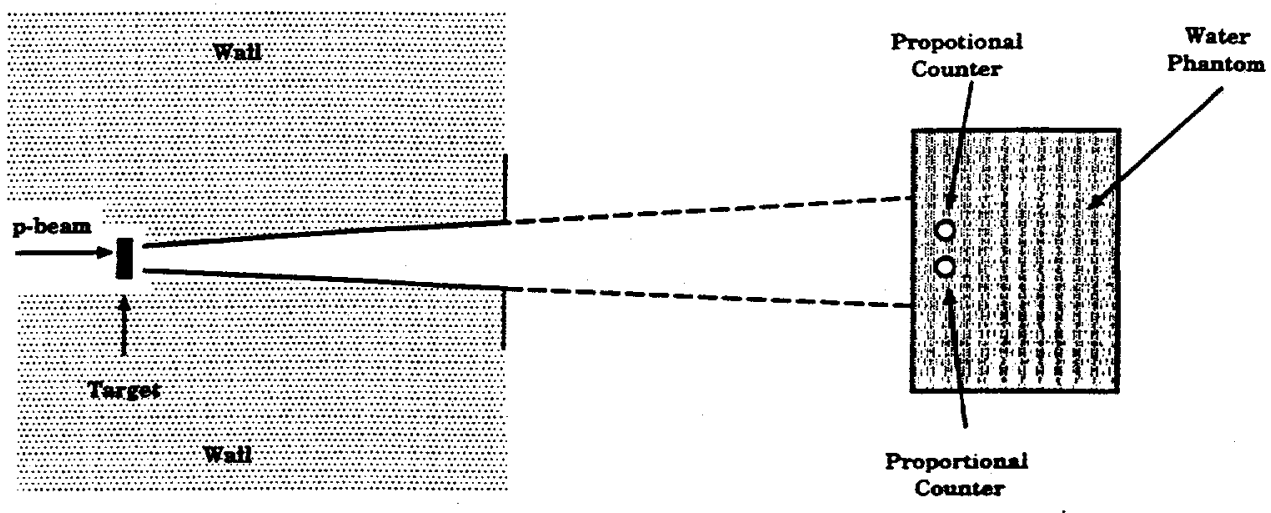

Figure 8: Typical proportional counter setup in the treatment room.

pulse heights had a relative range of one to 1000 . The pulser spectrum was also used to determine ADC and amplifier offsets. An $\alpha$ calibration spectrum was acquired before and after each run in order to determine changes in the counter performance.

For most data runs, two counters were irradiated simultaneously and were therefore arranged symmetrically about the beam axis at the isocenter distance of $1.80 \mathrm{~m}$. Figure 8 illustrates a typical setup of the proportional counters in the treatment room.

After pulse heights have been calibrated in terms of $\epsilon, D(\epsilon)$ is determined according to equation 3.21. Data were logarithmically binned into 40 bins per decade and displayed as $\epsilon D(\epsilon)$ vs. $\log \epsilon$. This display accommodates the large range of $\epsilon$ values while maintaining an equal area equal dose relationship. 


\subsection{Dose Rate Reductions}

Neutrons for patient therapy are delivered in $57 \mu$ s long macro beam pulses with a $15 \mathrm{~Hz}$ repetition rate. This beam structure is dictated by the booster synchrotron since the linac's primary function is that of a pre-accelerator for the booster. Beam is injected into the booster only for a fraction of time (3\%) and the linac beam is available for NTF during the remaining time. A macro pulse contains typically $1.4 \times 10^{13}$ protons. Within these beam pulses, the beam is divided into a $200 \mathrm{MHz}$ fine structure of micropulses. These numbers translate to a $0.043 \%$ duty cycle. The beam structure is illustrated in Figure 9: Considering that under normal patient treatment conditions, the average dose rate at $100 \mathrm{~mm}$ tissue equivalent liquid depth at isocenter is $6.7 \mathrm{mGy} / \mathrm{sec}$, the instantaneous dose rate within the $200 \mathrm{MHz}$ fine structure is $15.67 \mathrm{~Gy} / \mathrm{sec}$. This dose rate far exceeds the operational limit of microdosimetric proportional counters. Since the counter is operated in pulse mode the event rate in the counter needs to be such that the probability of pulse pile up is minimized. To achieve this the dose rate needs to be reduced by a factor of $10^{5}$.

The high instantaneous dose rate had thus previously inhibited microdosimetric measurements to be performed in the Fermilab beam. This project was triggered when Kroc [23] developed a technique to reduce the neutron dose rate by a sufficient amount. This technique consists of shortening of the $57 \mu s$ beam pulses designated for neutron therapy down to several tens of nanoseconds. At those short pulse durations the instantaneous dose rate is reduced by turning of the beam before it reached its full intensity. This is illustrated in Figure 10. Using this technique it is possible to obtain acceptable microdosimetric spectra. Unfortunately, this technique reduces the duty cycle even further and in order to obtain a pile-up minimized spectra a one event per second event rate has to be used. While it is possible to measure microdosimetric spectra using this technique, it is extremely 
painful, necessitating 80 hour runs.

Fortunately, during the course of this project, a second technique was developed by members of the Fermilab linac group, that allowed us to obtain pile-up minimized data at an event rate of ten events per second. In this technique the linac beam is deliberately defocused. The Cockroft-Walton pre-accelerator produces a $750 \mathrm{keV}$ continuous beam. Before entering the linac, this beam is formed into beam bunches by a buncher. In an effort to reduce the beam intensity this buncher is turned off. Furthermore, focusing magnets along the linac line are mistuned such that they now defocus the beam. The bending magnets that bend protons into the neutron therapy facility (NTF) beam line are unchanged. to ensure that only 66 $\mathrm{MeV}$ protons are extracted. The proton beam is now reduced in intensity during the whole $57 \mu s$ pulse duration. The only disadvantage of this technique is that by mistuning the linac beam, all downstream operations, i.e. the rest of Fermilab, is affected. In practice this means that microdosimetry measurements at NTF have to be restricted to times when all other Fermilab accelerators are off. During the last year there have been some extended maintenance periods in addition to a 10 day lab wide shut down that, in combination, allowed us to measure all data shown in this thesis using this improved technique. 


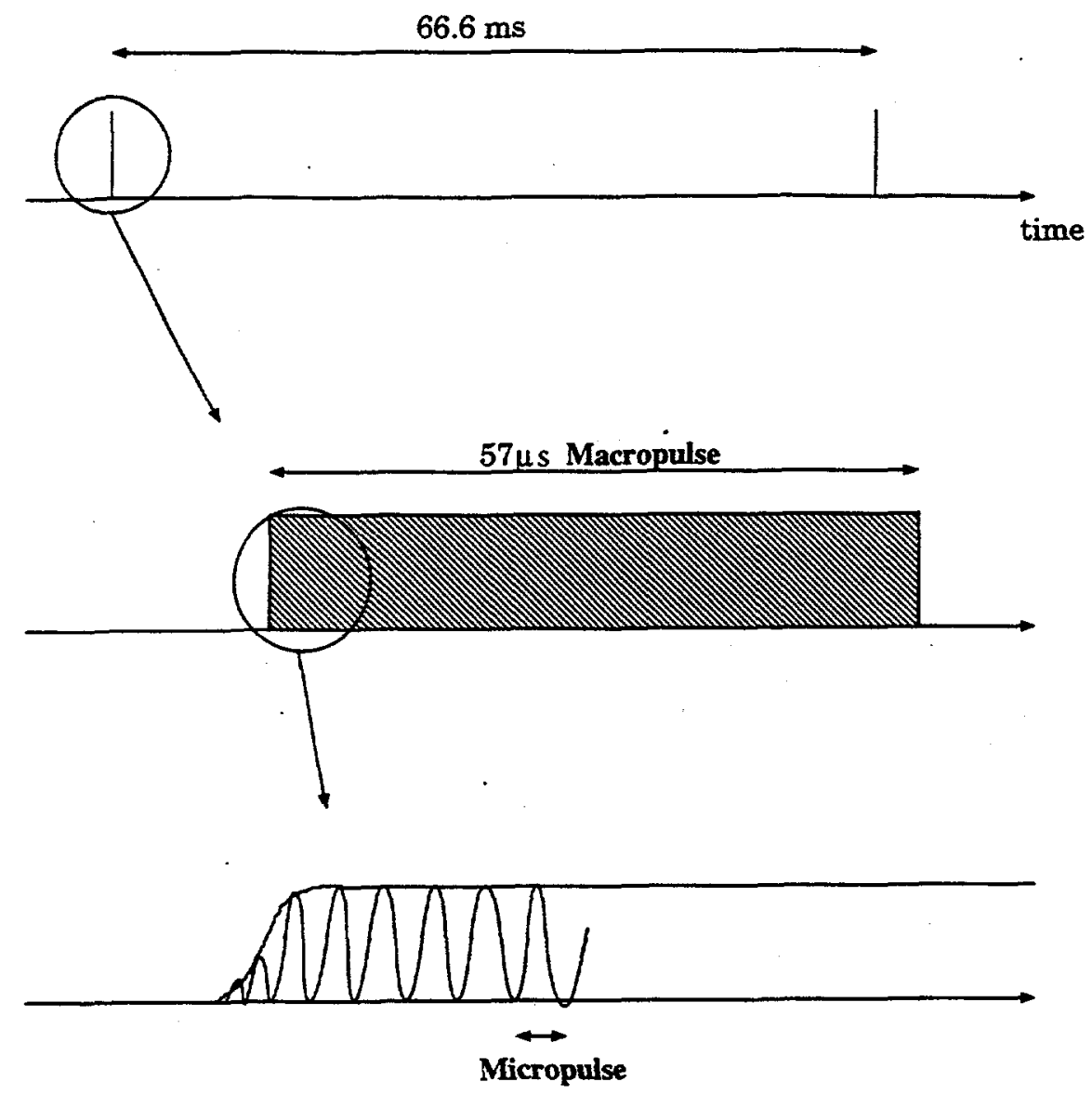

Figure 9: The linac beam structure.

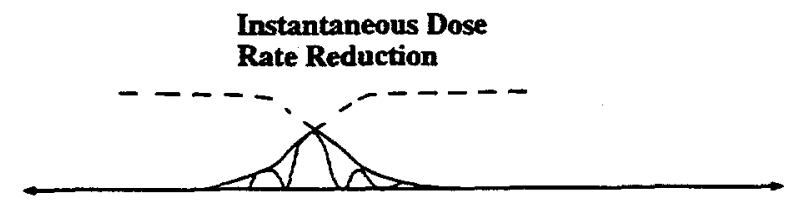

Figure 10: The modified beam structure, using Kroc's method [23]. 


\section{Chapter 5}

\section{Relative Carbon and Oxygen Kerma Factor Ratios}

\subsection{Introduction}

The quantity of interest in radiation therapy is the absorbed dose in tissue delivered during treatment. A determination of this quantity is, however. not trivial since it can not be directly measured. Commonly an ion chamber with a tissue equivalent plastic (A-150 Plastic) wall is used to mimic real tissue. In photon therapy, where the absorbed dose is a largely a function of the electron density of the material, this approach is valid since A-150 and tissue are matched in this quantity.

In neutron therapy, where cross sections can change drastically from one element to the next, the above method fails. Ideally a tissue mimicking plastic should be matched exactly in elemental composition to the tissue that is being mimicked. But this is hard to achieve due to the difficulty of constructing a plastic with such a high oxygen content and due to the need for a higher carbon content in the plastic in order for it to be conducting. Even though the exact elemental composition of tissue depends on the tissue type, ICRU muscle tissue can be used to represent most soft tissues. Table 4 lists the elemental compositions of ICRU muscle tissue and A-150 plastic. As can be seen the two materials mainly differ in carbon and oxygen content. The absorbed dose measured in A-150 needs to be corrected for this discrepancy to determine the absorbed dose in ICRU tissue. Since the carbon kerma is larger than the oxygen kerma for neutron energies used in therapeutic beams, the A-150 kerma is larger than the ICRU tissue kerma for these beams. 


\begin{tabular}{|c|c|c|}
\hline Element & ICRU Tissue & A-150 Plastic \\
\hline \hline $\mathrm{H}$ & 10.2 & 10.1 \\
\hline $\mathrm{C}$ & 12.3 & 77.6 \\
\hline $\mathrm{N}$ & 3.5 & 3.5 \\
\hline $\mathrm{O}$ & .72 .9 & 5.3 \\
\hline other & 1.1 & 3.5 \\
\hline
\end{tabular}

Table 4: Elemental composition (in \% weight) of ICRU muscle tissue and A-150 tissue equivalent plastic [35, 53].

Furthermore, the carbon to oxygen kerma ratio varies with neutron energy.

\subsection{Correction of A-150 Reading}

The kinetic energy released in matter, KERMA, is the amount of kinetic energy given to charged particles by indirectly ionizing radiation per unit mass. If charged particle equilibrium (CPE) is established, kerma $\mathrm{K}$ is numerically equal to the absorbed dose $\mathrm{D}$. Kerma factors are used to calculate the kerma in a given material.

$$
K=\Phi(E) \times k_{\phi, j}(E)[J / k g]
$$

Where $k_{\phi, j}$ is the kerma factor which depends on the indirectly ionizing particle energy $E$ and the element $j$ it interacts with, and $\Phi(E)$ is the fluence of indirectly ionizing particles with energy $E$. The kerma factor for a compound is the sum over all the mass fractions $w_{j}$ multiplied by their kerma factor values.

$$
k_{\phi, c o m p .}(E)=\sum_{j} w_{j} \times k_{\phi, j}(E)\left[G y m^{2}\right]
$$

In a given beam, the tissue to A-150 absorbed dose ratio, $D_{t} / D_{a}$ is, under $\mathrm{CPE}$ conditions, equal to the corresponding kerma factor ratio. This ratio can be derived in the following manner, 


$$
\begin{aligned}
k_{\phi, t}=k_{\phi, a} & -w_{(\text {in } a)} \times k_{\phi, o}+w_{(\text {in } t)} \times k_{\phi, o} \\
& -w_{(c \text { in } a)} \times k_{\phi, c}+w_{(\text {in } t)} \times k_{\phi, c} \quad\left[G y m^{2}\right]
\end{aligned}
$$

dividing by the A-150 kerma factor yields,

$$
\begin{aligned}
\frac{k_{\phi, t}}{k_{\phi, a}}=1 & +\left(w_{(o \text { in } t)}-w_{(\text {in } a)}\right) \times \frac{k_{\phi, o}}{k_{\phi, a}} \\
& +\left(w_{(c \text { in } t)}-w_{(c \text { in } a)}\right) \times \frac{k_{\phi, c}}{k_{\phi, a}}
\end{aligned}
$$

For a broad energy spectrum, the effective kerma factor ratio is

$$
\begin{aligned}
\frac{k_{\phi, t}}{k_{\phi, a}}=1 & +\left(w_{(\text {o in } t)}-w_{(o \text { in } a)}\right) \times \int \frac{k_{\phi, o}}{k_{\phi, a}} d E \\
& +\left(w_{(c \text { in } t)}-w_{(c \text { in } a)}\right) \times \int \frac{k_{\phi, c}}{k_{\phi, a}} d E
\end{aligned}
$$

where $w_{(j \text { in } k)}$ are the weight fractions of element $j$ in material $k$ and subscripts $t$ and a indicate ICRU tissue and A-150 plastic, respectively.

This calculation requires not only knowledge of the carbon and oxygen kerma factors relative to A-150 plastic kerma factors over the whole energy range but also knowledge of the neutron energy spectrum.

While extensive nuclear data are available for neutron interactions up to 15$20 \mathrm{MeV}$, only sparse data exist at higher neutron energies. Kerma factors for carbon $[7,51]$, oxygen $[7,18]$ and A-150 plastic [51] have been reported for some neutron energies above $20 \mathrm{MeV}$.

In order to supplement the sparse experimental data a variety of calculations have been performed. Recently, ICRU tissue and A-150 kerma have been calculated for neutron energies ranging up to $100 \mathrm{MeV}$ [11]. These data are based on nuclear model calculations that took experimental data into consideration, when possible. 


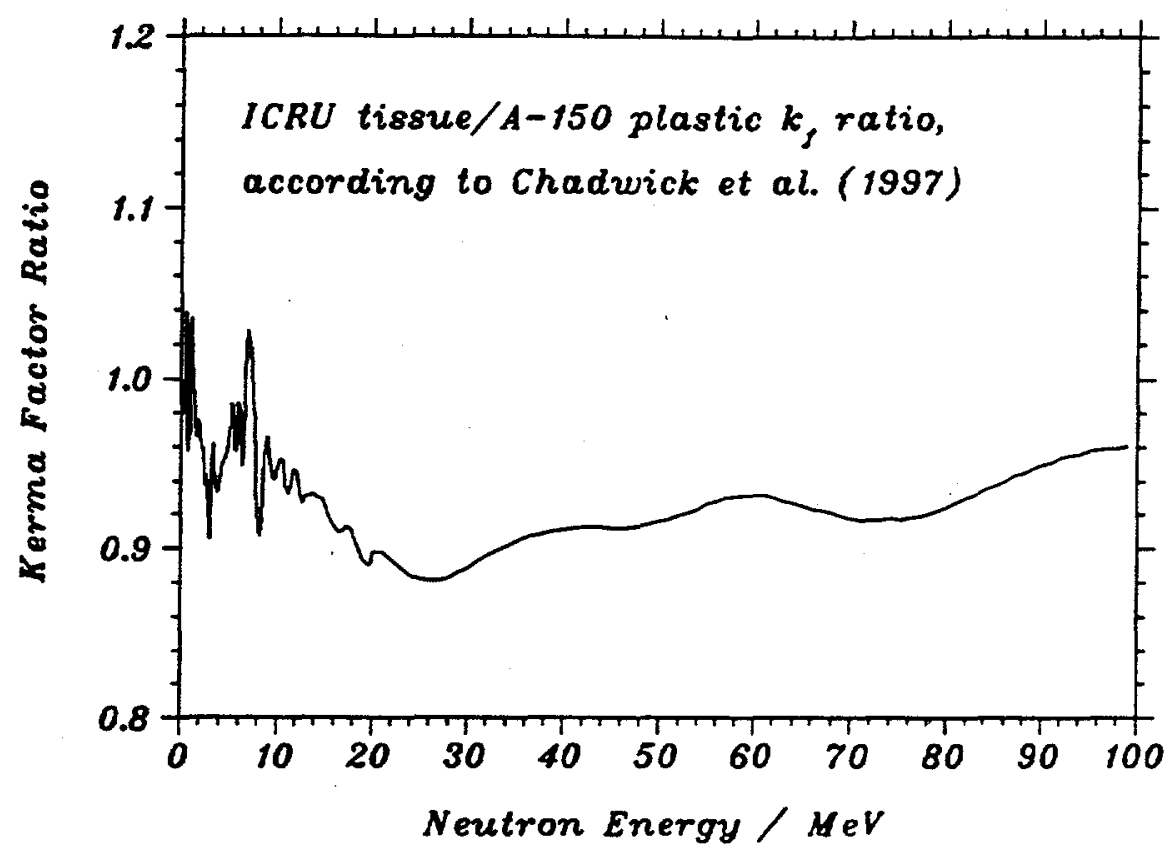

Figure 11: Calculated ICRU tissue to A-150 plastic kerma factor ratio. Kerma factors were taken from Chadwick et al. [11].

The ICRU tissue to A-150 plastic kerma factor ratios versus neutron energy which were calculated using this data are shown in Figure 11.

Schrewe et al. [50] recently published A-150 plastic to ICRL tissue kerma conversion factors for neutron energies up to $100 \mathrm{MeV}$ which had been calculated from published data. A conversion factor of $0.92 \pm 0.02$ for neutron energies above 20 $\mathrm{MeV}$ is recommended in this publication.

After reviewing published neutron spectra data and available kerma factors Awschalom et al. [2] deduced a value of $0.93 \pm 0.03$ for the tissue to A-150 kerma for a $\mathrm{p}(66) \mathrm{Be}$ neutron beam. Ross et al. [45] used Monte Carlo transport codes to calculate the neutron fluence spectrum at Fermilab's neutron therapy facility. This calculated spectrum is shown in figure 12 . By weighting the calculated spectra with published kerma factor values, he deduced an A-150 plastic to ICRU tissue kerma ratio of 0.93 . 


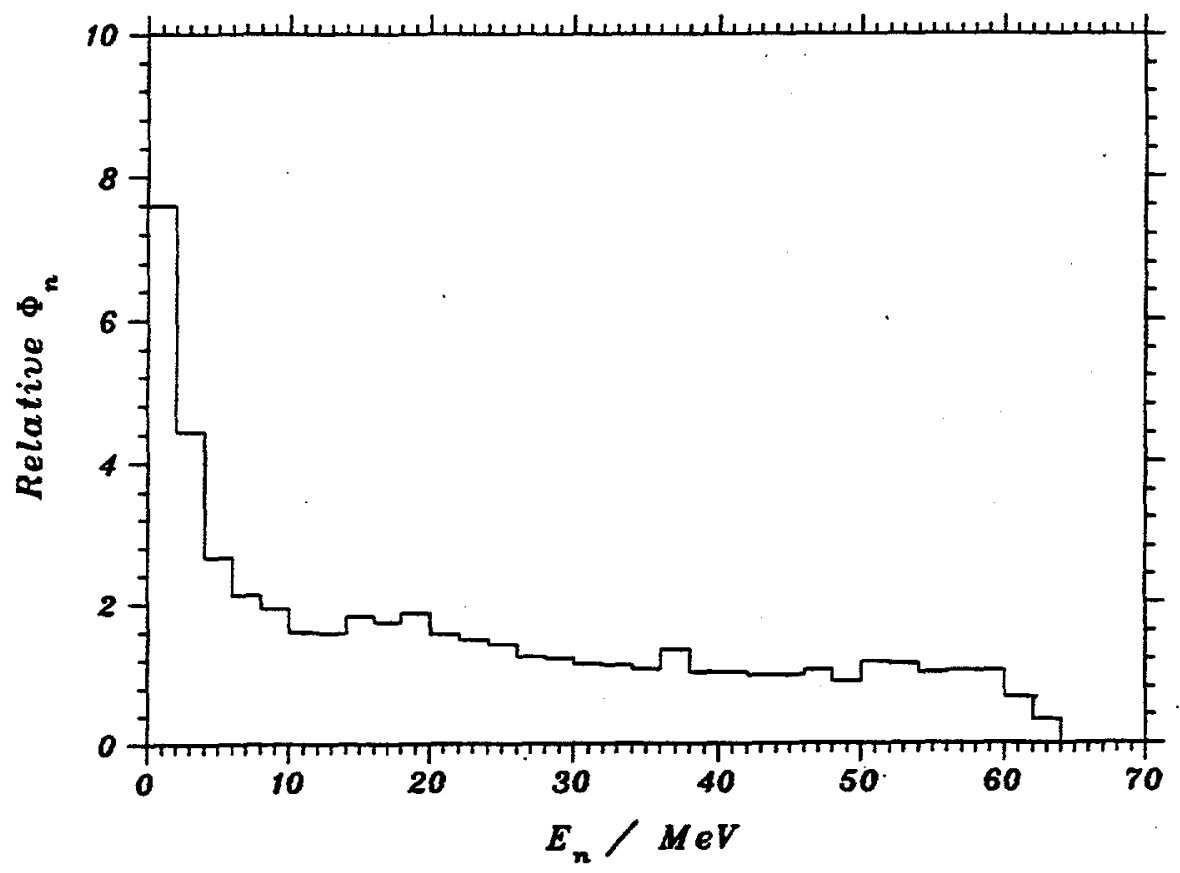

Figure 12: Calculated neutron fluence spectrum for NTF [45].

The current dosimetry protocol [34] for fast neutron beams recommends a value of 0.95 to be used for the conversion from A-150 plastic kerma to ICRU tissue kerma in the NTF beam.

\subsection{Measurements}

Alternatively we propose to obtain the effective carbon to A-150 and oxygen to A-150 kerma factors for the broad energy neutron beam by direct measurements. If two proportional counters are irradiated simultaneously in the same neutron beam, that is they both are exposed to the same neutron energy spectrum and fluence, the absorbed dose ratio of the counters is then equal to the kerma ratio and hence to the effective kerma factor ratio. This method eliminates the need to know the kerma factor ratios for relevant neutron energies as well as the need to know the neutron energy spectrum. 
Three measurements with two counters each were performed in order to determine the A-150 to tissue kerma factor ratio. An A-150 counter was simultaneously irradiated with carbon, zirconium and zirconium oxide counters. It is assumed that when two counters are arranged symmetrically about the beam axis, they are exposed to the same neutron fluence. From the A-150/carbon detector pair measurement the corresponding kerma factor ratio can be directly determined. For the determination of oxygen kerma two counters are needed since a detector wall cannot be constructed out of oxygen. For this reason two counters which differ in oxygen concentration of the wall, namely a $\mathrm{Zr}$ and $\mathrm{ZrO}_{2}$ counter, were constructed and the relative oxygen kerma can be determined by a subtraction technique [12].

The above set of measurements was made in a $10 \times 10 \mathrm{~cm}^{2}$ field size beam at the isocenter distance of $1.8 \mathrm{~m}$. Counters were arranged symmetrically about the beam axis and were irradiated free in air. In order to establish full build-up conditions the A-150 plastic counter wall (thickness: $12.7 \mathrm{~mm}$ ) was supplemented with a $16 \mathrm{~mm}$ thick Nylon 6 build-up cap. Nylon 6 can be substituted for A-150 plastic as a build-up material [6]. For the carbon counter a $6 \mathrm{~mm}$ carbon build-up cap was used in addition to the counter wall thickness of $12.7 \mathrm{~mm}$. The total carbon thickness is equivalent to the total A-150 and Nylon thickness in terms of proton range. No build-up caps were used on the $\mathrm{Zr}$ and $\mathrm{ZrO}_{2}$ counters.

\subsection{Results}

The acquired carbon, zirconium and zirconium oxide data are shown in Figures $13,14,15$. A microdosimetric spectrum measured in a Cs-137 source field is used to represent the microdosimetric spectrum due to the gamma contamination in a neutron beam. Hence the measured spectra were corrected for gamma contamination by fitting a gamma spectrum (Cs-137) to the measured spectra at event sizes 


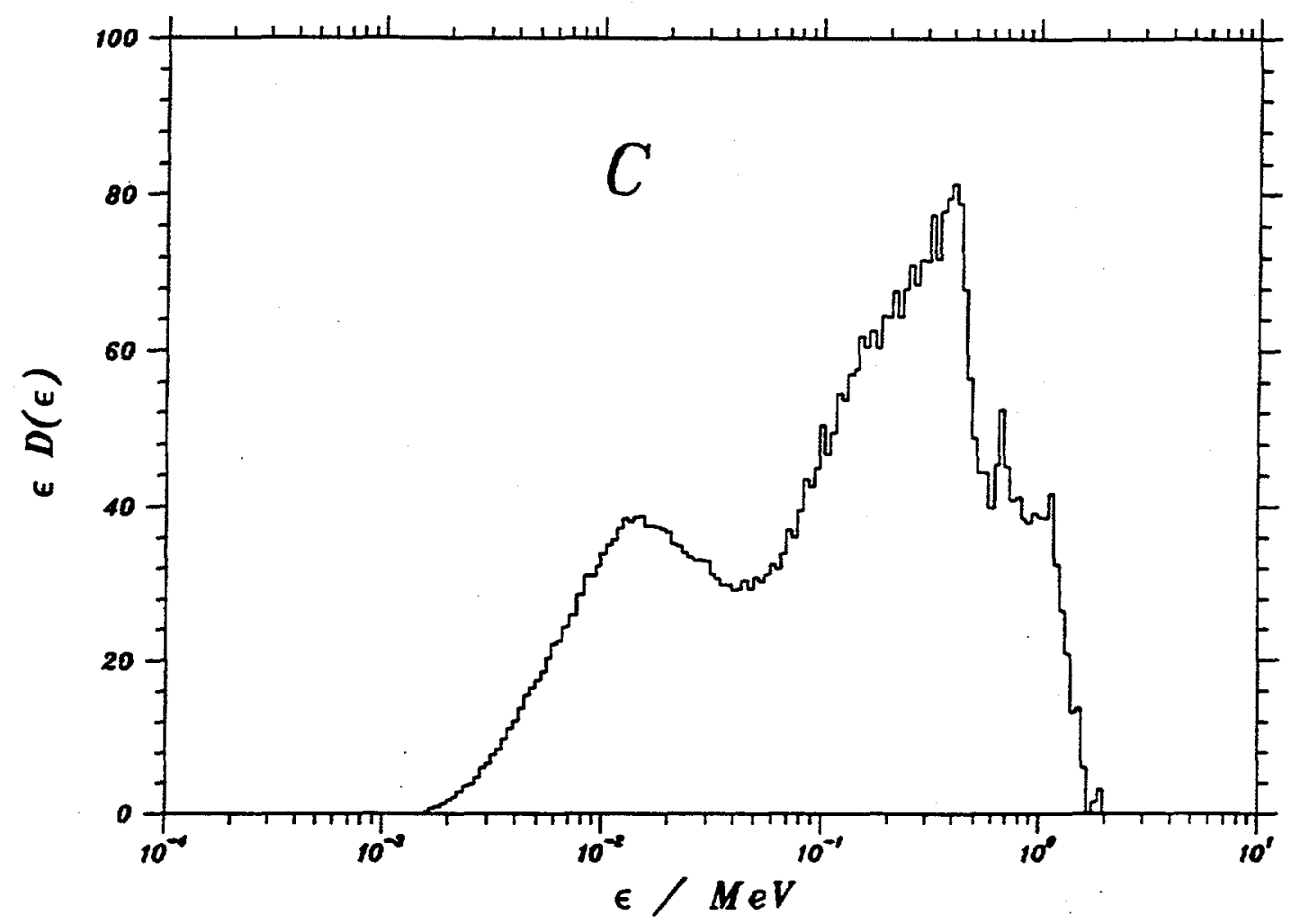

Figure 13: Carbon microdosimetry data.

$\epsilon=0.001$ to $0.0013 \mathrm{MeV}$ and subsequently the gamma component was subtracted from the measured event size spectra. The remaining event size spectrum is then only due to the neutron component of the beam. The spectra shown were normalized to unit dose.

Gas doses measured with each counter and calculated wall kermas are listed in table 5. In order to obtain wall kerma, gas doses were multiplied with $r_{m, g}$ values taken from Newhauser et al. [32]. The $r_{m, g}$ values used were those for isobutane based tissue equivalent gas and $25 \mathrm{MeV}$, which is estimated to be the the average neutron energy of the NTF beam. Wall kermas were used to determine the effective carbon and oxygen to A-150 tissue equivalent plastic kerma factor ratios.

Carbon to A-150 TE plastic kerma factor ratio: Results obtained from 


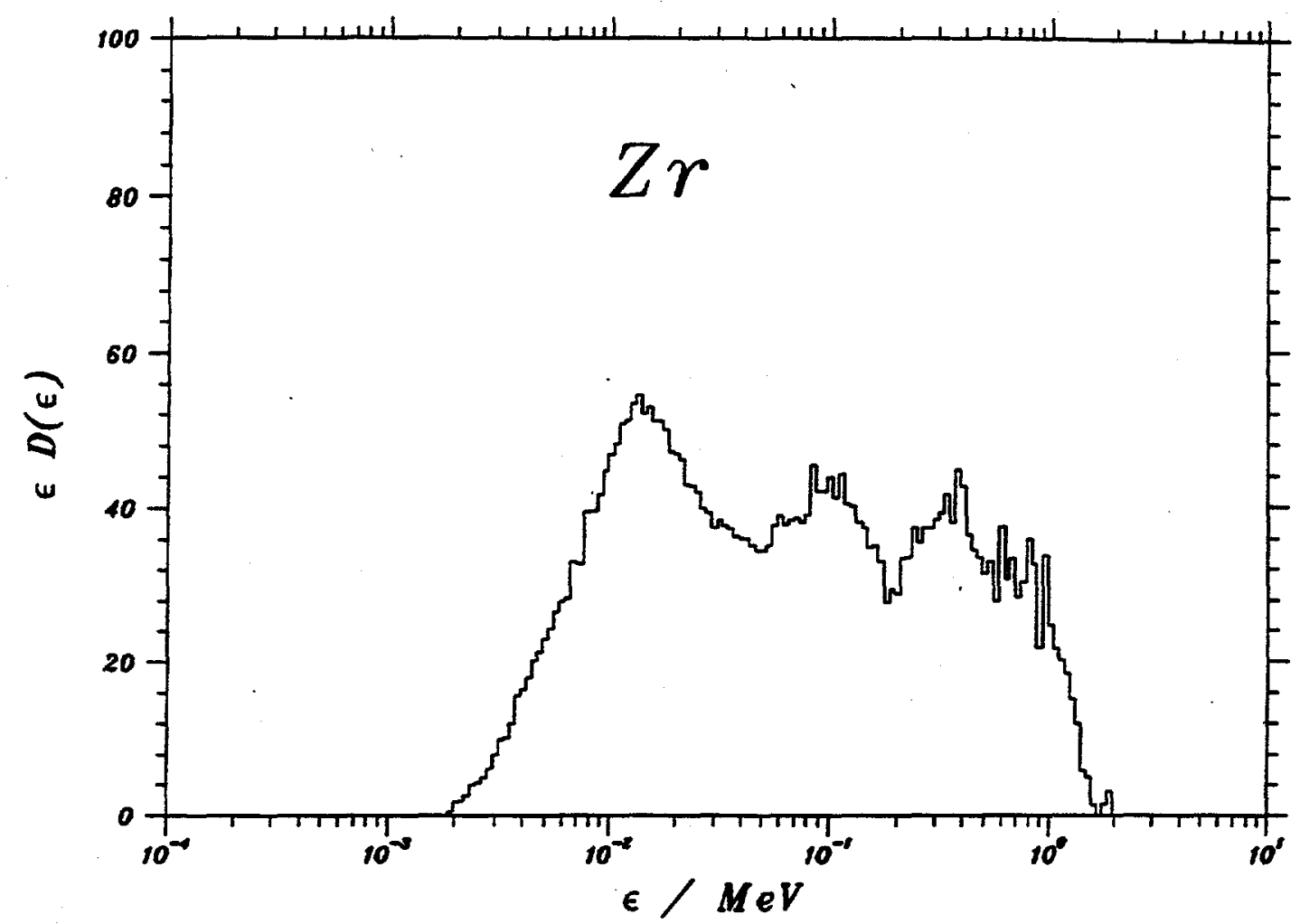

Figure 14: Zirconium microdòsimetry data.

\begin{tabular}{|c|c|r|r|r|}
\hline Run & Counter & Gas dose [mGy] & $r_{m, g}$ value & Wall kerma [mGy] \\
\hline \hline$C / T E$ & Carbon & $13.77 \pm 1.52$ & $0.830 \pm 0.03$ & $11.43 \pm 1.33$ \\
& A-150 & $21.52 \pm 1.25$ & $1.001 \pm 0.04$ & $21.54 \pm 1.52$ \\
\hline$Z r / T E$ & Zirconium & $4.40 \pm 0.27$ & $0.442 \pm 0.02$ & $1.95 \pm 0.15$ \\
& A-150 & $27.00 \pm 1.40$ & $1.001 \pm 0.04$ & $27.03 \pm 1.77$ \\
\hline$Z r O_{2} / T E$ & Zirconium oxide & $5.60 \pm 0.47$ & $0.488 \pm 0.02$ & $2.73 \pm 0.26$ \\
& A-150 & $17.62 \pm 1.03$ & $1.001 \pm 0.04$ & $17.64 \pm 1.25$ \\
\hline
\end{tabular}

Table 5: Intermediate results for the ICRU tissue to A-150 kerma factor ratio determination. 


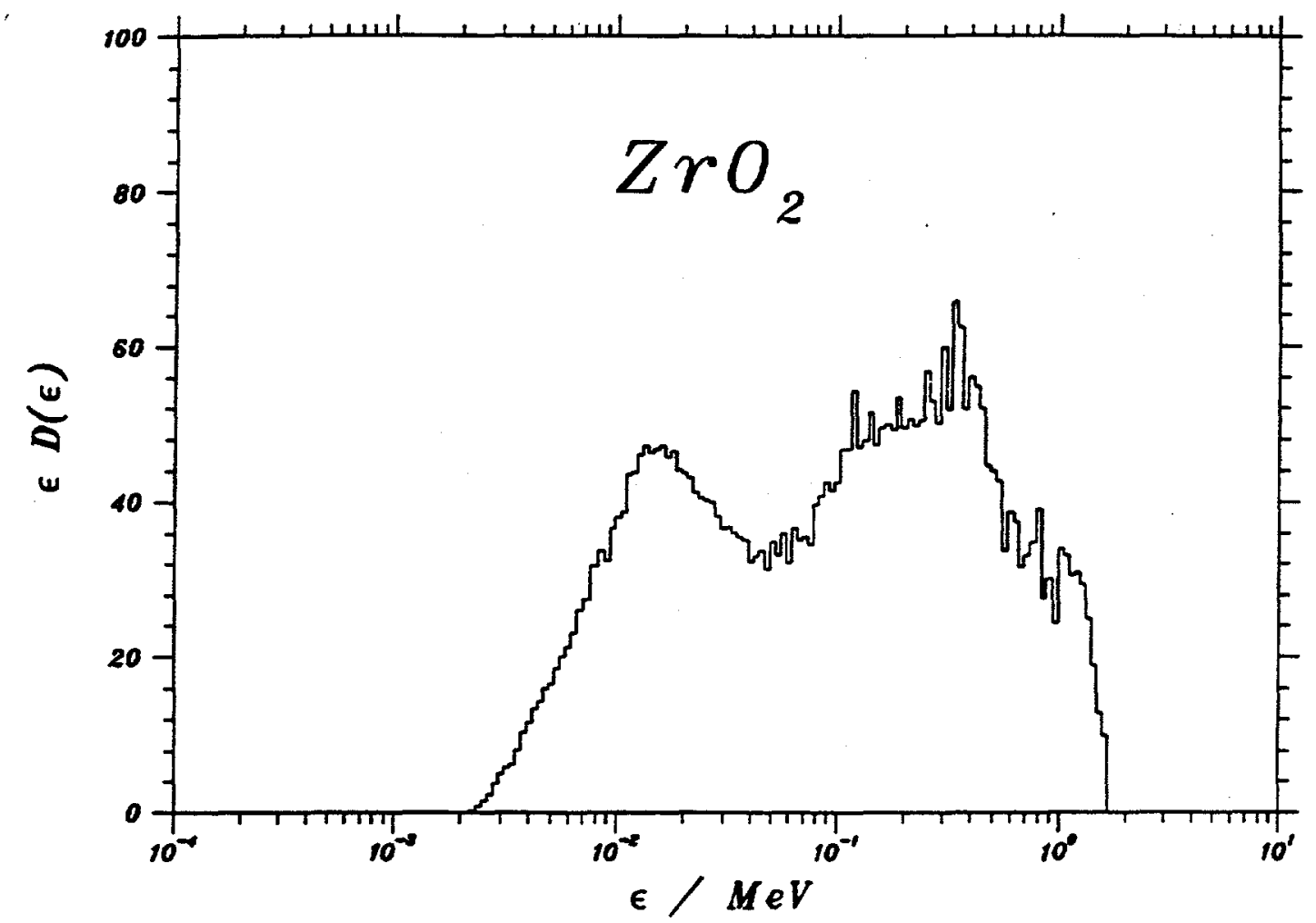

Figure 15: Zirconium oxide microdosimetry data. 
the C/A-150 run were used to determine the effective kerma factor ratio. The absorbed gas dose in the carbon counter was $13.77 \pm 1.52 \mathrm{mGy}$. The uncertainty is due to a $10 \%$ uncertainty in counting statistics, $3 \%$ uncertainty due to calibration procedure, $3 \%$ uncertainty due to the use of a constant $\frac{\bar{W}}{e}$ value and $2 \%$ uncertainty due to invalid Bragg-Gray condition. The gas dose was multiplied with a $r_{m, g}$ value of $0.83 \pm 0.03$ to compute a wall kerma of $11.43 \pm 1.32 \mathrm{mGy}$. The gas dose in the A-150 counter was $21.52 \pm 1.25 m G y$. The uncertainty was calculated to be $6 \%$, due to $4 \%$ uncertainty in counting statistics, $3 \%$ due to calibration procedure and $3 \%$ uncertainty in the use of a constant $\frac{\bar{W}}{e}$ value. A $r_{m, g}$ value of $1.001 \pm 0.04$ was used for all A-150 counters. The A-150 wall kerma was calculated to be $21.54 \pm 1.52 m G y$. The carbon to A-150 kerma factor ratio was calculated to be $0.53 \pm 0.07$.

Oxygen to A-150 TE plastic kerma factor ratio: In order to determine the effective oxygen to A-150 kerma factor ratio a subtraction technique has to be employed. The $\mathrm{Zr}$ and $\mathrm{ZrO}_{2}$ to A-150 TE plastic kerma factor ratios were determined analogously to the determination of the carbon to A-150 kerma factor ratio. Values of $0.072 \pm 0.007$ and $0.155 \pm 0.018$ were calculated for the $Z r$ and $\mathrm{ZrO}_{2}$ to A-150 kerma factor ratios, respectively.

The zirconium oxide kerma is the summation of zirconium and oxygen kerma weighted by their mass fraction.

$$
K_{Z r O 2}=w_{O} \times K_{O}+w_{Z r} \times K_{Z r}[G y]
$$

For $\mathrm{ZrO}_{2}$ the oxygen mass fraction $w_{0}$ is 0.26 , the zirconium mass fraction $w_{Z r}$ is 0.74 . The oxygen kerma $K_{O}$ is then,

$$
K_{O}=\frac{K_{Z r O 2}-0.74 \times K_{Z r}}{0.26}[G y]
$$

Using this equation an effective oxygen to A-150 kerma ratio was computed to be 


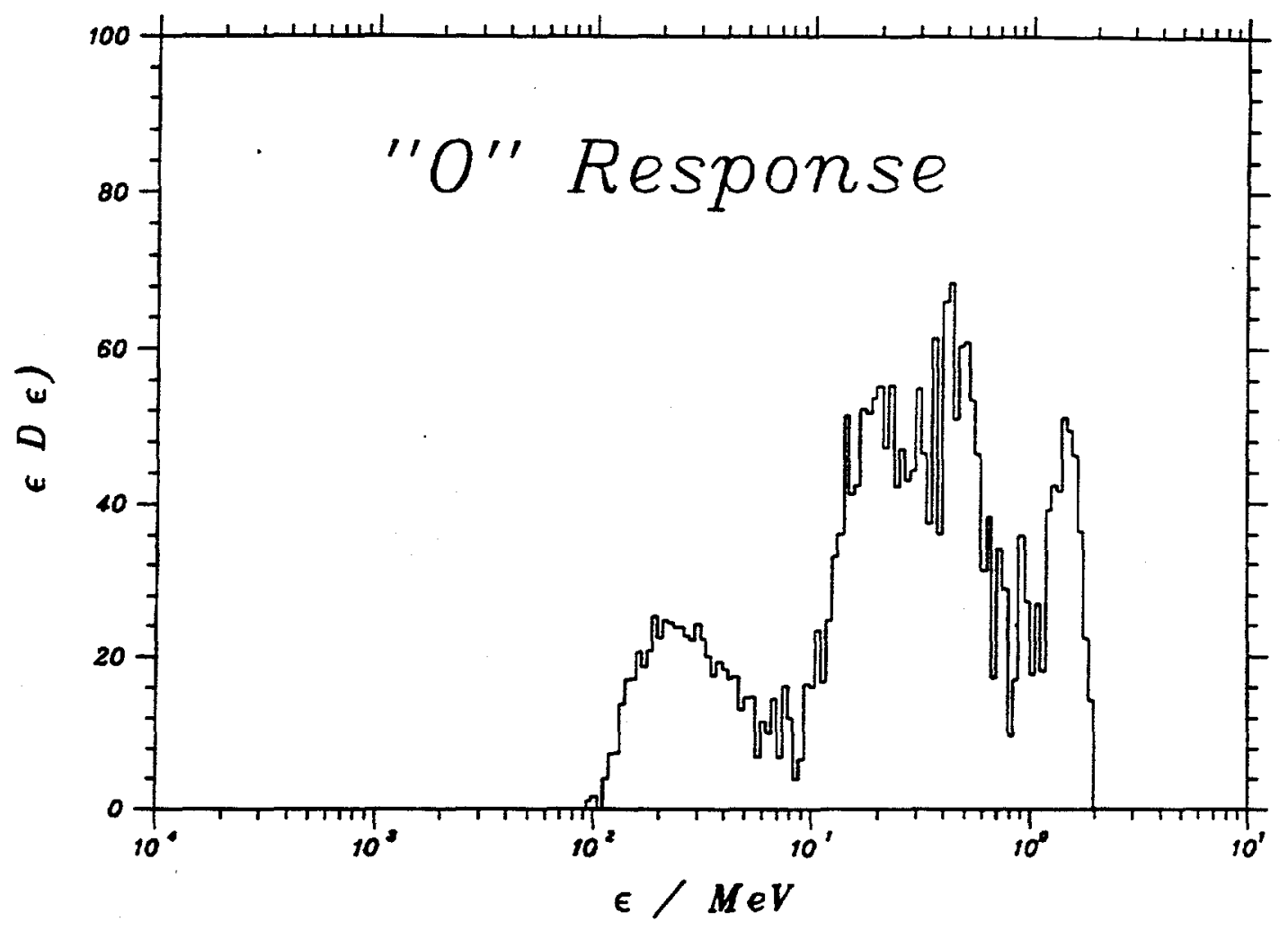

Figure 16: "Oxygen" response microdosimetry data.

$0.39 \pm 0.02$. Figure 16 shows the oxygen microdosimetry data that were obtained by subtracting the weighted $\mathrm{Zr}$ spectrum from the $\mathrm{ZrO}_{2}$ spectrum.

ICRU tissue to A-150 tissue equivalent plastic kerma factor ratio: For the determination of the effective ICRU tissue to A-150 plastic kerma ratio, equation 5.31 was used, where $w_{(O \text { in } T)}$ is $0.729, w_{(O \text { in } A)}$ is $0.053, w_{(C \text { in } T)}$ is 0.123 and $w_{(C \text { in } T)}$ is 0.776 . The ICRU tissue to A-150 plastic kerma factor ratio is then

$$
\frac{k_{T}}{k_{A}}=0.92 \pm 0.04
$$




\subsection{Discussion}

The result obtained through measurements agrees well with previous results calculated for the NTF beam. Both, Ross et al. [45] and Awschalom et al. [2] recommend an ICRU tissue to A-150 kerma factor ratio of 0.93 which agrees well with our result of $0.92 \pm 0.04$. Ross et al. [45] used data from Chadwick et al. [11] to compute the ICRU tissue to A-150 kerma factor ratio.

The measured carbon to A-150 kerma factor value of $0.53 \pm 0.07$ for the NTF beam compares well with with a determination of the same quantity by Schrewe et al. [51]. They measured a value of $0.51 \pm 0.06$ for the carbon to A-150 kerma factor ratio at a neutron energy of $44.5 \mathrm{MeV}$.

Our calculations also yields the effective carbon to oxygen kerma factor ratio of $1.36 \pm 0.19$ for the NTF beam. This value equals the carbon to oxygen kerma factor ratio calculated by Chadwick et al. [11] for $35 \mathrm{MeV}$.

The current value in use at NTF for the conversion of A-150 to ICRU tissue dose is 0.95 , as recommended by the AAPM protocol [34]. As a result, NTF doses could be 2-3\% lower than prescribed, assuming a true conversion factor of 0.92-0.93. 


\section{Chapter 6}

\section{Beam Characterization}

\subsection{Introduction}

It is well established that for the same absorbed dose neutrons are more effective than photons in causing a biological effect. This is quantified by the radiobiological effectiveness (RBE) of the beam which is defined as

$$
R B E(\text { neutron })=\frac{\text { Dose }(250 \mathrm{keV} x-\text { rays })}{\text { Dose (neutron })}
$$

where Dose (250 keV $x$-rays) and Dose (neutron) are to result in the same amount of biological effect [16]. The higher RBE of neutrons is attributed to densely ionizing secondary particles. Fast neutron therapy beams have a typical RBE value of three to four for fractionated therapy.

In a typical neutron therapy beam most absorbed dose is deposited by secondary protons generated in the neutron beam. The ionization density, or stopping power, distribution of protons in a fast neutron beam is, on average, an order of magnitude larger than the stopping power distribution of electrons generated in a Co-60 beam.

The RBE, as a function of the linear energy transfer coefficient (LET), i.e. the energy lost by the particle per unit path length, rises with increasing LET. The function peaks at a LET values of $100 \mathrm{keV} / \mu \mathrm{m}$ before it decreases with increasing LET values [3].

The stopping power of protons in tissue peaks at a value of just below $100 \mathrm{keV} / \mu \mathrm{m}$ at a proton energy of $75 \mathrm{keV}$. Any higher and lower energy proton has 
a lesser RBE. The average secondary proton energy generated in tissue increases with the neutron beam energy. An increase in the average fast neutron beam energy slightly decreases the RBE of the beam. Considering that no neutron therapy facility is identical to another in terms of neutron energy spectra, each facility has its own unique $R B E$ value.

Beauduinet al. [4] performed a radiobiological study to determine the RBE variations between several neutron beams. Their results show that the RBE of neutron beams decreases as the half value thickness, or energy, of the beams increases. For a neutron beam generated by $20 \mathrm{MeV}$ deuteron bombardment of a beryllium target, the RBE was measured to be $50 \%$ larger than the RBE of a $\mathrm{p}(65)$ Be beam.

Besides the primary neutron energy spectrum, several other parameters influence the neutron energy spectrum. The beam collimation as well as field shaping devices all influence the neutron spectrum that eventually is used to irradiate the patient. Consequently all these parameters influence the RBE of the treatment beam to some degree.

As an alternatively, to radiobiological experiments, microdosimetry can be used to map RBE variations in a neutron beam by weighting the measured event size distribution with biological weighting functions (section 3.8). Whereas the saturation corrected mean lineal energy $y^{*}$ (or $\epsilon^{*}$ ) can be used to approximate RBE variations, it neglects the RBE dependence on biological endpoint [40]. The empirical weighting function determined by Pihet et al. [41] was derived from radiobiological data on early effects in mice, and is thus inherently valid only for that specific endpoint.

We investigated qualitative changes in the NTF beam with depth and field size by obtaining microdosimetric spectra under various irradiation conditions. All spectra were then weighted with the $\epsilon_{\text {sat }}$ and Pihet's r-functions. Relative changes in $\epsilon^{*}$ and $\mathrm{R}$ were determined. 


\subsection{RBE variations with depth in tissue}

As a broad spectrum neutron beam penetrates into tissue it hardens due to the preferential absorption of lower energy neutrons. The RBE of the neutron beam therefore decreases with depth in tissue. Several studies on this subject verified a reduction in RBE with depth in neutron beams. Hall et al. [17] measured a $10 \%$ reduction in RBE in a $\mathrm{p}$ (43)Be neutron beam between $20 \mathrm{~mm}$ and $120 \mathrm{~mm}$ using Chinese hamster V79 cells. Later, a group at Clatterbridge found the same RBE reduction in the $p(62)$ Be beam [20] using mouse intestinal crypt cells.

A study of this effect in the $\mathrm{p}(66) \mathrm{Be}(40)$ neutron therapy beam at the National Accelerator Center (NAC) at Faure, South Africa [j2] using microdosimetry, yielded a reduction of $6 \%$ in $y^{*}$ at $150 \mathrm{~mm}$ water depth as compared to $25 \mathrm{~mm}$ depth. The effects of various thickness polyethylene filters were subsequently studied. Polyethylene filtration reduces the variation of $y^{*}$ with depth by hardening the beam in the filter material, therefore reducing the hardening effect in the patient. In a $50 \mathrm{~mm}$ polyethylene filtered beam, the above $y^{*}$ variation was reduced to zero, however such a thick filter also reduces the dose rate to an unacceptable low level. This study led to the permanent installation of a $25 \mathrm{~mm}$ thick polyethylene filter in the Faure beam. In this beam a $5 \%$ reduction in $y^{*}$ between $25 \mathrm{~mm}$ and $200 \mathrm{~mm}$ depth was subsequently measured [52].

It should be noted that the South African beam and Fermilab's beam are both produced by $66 \mathrm{MeV}$ protons on beryllium. However, the RBE changes with depth are expected to be larger in the Fermilab beam due to the thicker beryllium target and the lack of a polyethylene filter in the Fermilab beam.

Hill et al. [19] performed radiobiological experiments in the Fermilab neutron therapy beam with Chinese hamster V79 cells. A RBE reduction of $10-15 \%$ was found between $30 \mathrm{~mm}$ and $240 \mathrm{~mm}$ depth in a water phantom. 


\subsection{RBE variations with field size}

As the field size is increased the amount of scattering in the collimation system increases. This enhances the lower energy component of the spectra, which influences the quality of the beam. RBE changes with field size in the South African beam have been determined by Binns [6]. The RBE of the beam was found to increase with field size $\left(30 \mathrm{~cm}^{2}\right.$ to $\left.400 \mathrm{~cm}^{2}\right)$ by $3-5 \%$ percent.

\subsection{Measurements}

An A-150 tissue equivalent plastic counter was used to measure microdosimetric spectra at different depths and field sizes. The counter was immersed in a $300 \mathrm{~mm} \times$ $300 \mathrm{~mm} \times 300 \mathrm{~mm}$ water filled phantom. The surface of the water phantom was placed at isocenter distance. The A-150 counter was positioned in the center of the radiation field. For three field sizes $(50 \mathrm{~mm} \times 50 \mathrm{~mm}, 100 \mathrm{~mm} \times 100 \mathrm{~mm}, 200 \mathrm{~mm} \times$ $200 \mathrm{~mm}$ ) spectra were acquired at three water depths $(50 \mathrm{~mm}, 100 \mathrm{~mm}, 150 \mathrm{~mm}$ ).

\subsection{Results}

The measured A-150 spectra were weighted with two biological weighting functions, $\epsilon_{\text {sat }}$ and Pihet's $r(\epsilon)$-function. These functions can be seen in figures 5 and 6 . After absolute $\epsilon^{*}$ values were determined, these values were normalized to the $\epsilon^{*}$ value obtained at a field size of $100 \mathrm{~mm} \times 100 \mathrm{~m}$ and a depth of $100 \mathrm{~mm}$. Since the $r(\epsilon)-$ function was derived in order to provide a parameter that allows the comparison of different neutron therapy facilities in terms of RBE, the absolute $R$-value for one measurement as well as relative $R$-values for all other measurements were calculated. The absolute $R$-value is normalized to the $p(65)$ beam at Louvainla-Neuve per definition. For the $100 \mathrm{~mm} \times 100 \mathrm{~mm}$ field size, $100 \mathrm{~mm}$ depth measurement a R-value of $1.08 \pm 0.04$ was calculated. All other results are listed 


\begin{tabular}{|c|c|c|c|}
\hline Field size in $\mathrm{mm}^{2}$ & Depth in $\mathrm{mm}$ & relative $\epsilon^{*}$ value & relative $R$-value \\
\hline \hline $50 \times 50$ & 50 & $1.01 \pm 0.01$ & $1.00 \pm 0.01$ \\
$50 \times 50$ & 100 & $0.97 \pm 0.01$ & $0.95 \pm 0.01$ \\
$50 \times 50$ & 150 & $0.95 \pm 0.01$ & $0.92 \pm 0.01$ \\
\hline \hline $100 \times 100$ & 50 & $1.04 \pm 0.01$ & $1.05 \pm 0.01$ \\
$100 \times 100$ & 100 & $1.00 \pm 0.01$ & $1.00 \pm 0.01$ \\
$100 \times 100$ & 150 & $0.97 \pm 0.01$ & $0.96 \pm 0.01$ \\
\hline \hline $200 \times 200$ & 50 & $1.04 \pm 0.01$ & $1.07 \pm 0.01$ \\
$200 \times 200$ & 100 & $1.01 \pm 0.01$ & $1.02 \pm 0.01$ \\
$200 \times 200$ & 150 & $0.97 \pm 0.01$ & $0.97 \pm 0.01$ \\
\hline
\end{tabular}

Table 6: $\epsilon^{*}$ and $R$ values relative to those obtained at a field size of $100 \mathrm{~mm} \times$ $100 \mathrm{~mm}$ and depth of $100 \mathrm{~mm}$.

in table 6.

Uncertainties in $\epsilon^{*}$ are due to counting statistics (1\%). The uncertainty in the absolute $R$-value is due to counting statistics (1\%) and due to an uncertainty in the R-function (4\%) [41]. However, relative R-values have an uncertainty of only $1 \%$ which is due to counting statistics only.

Variations with depth: Figures 17, 18, 19 show A-150 spectra measured at field sizes of $50 \mathrm{~mm} \times 50 \mathrm{~mm}, 100 \mathrm{~mm} \times 100 \mathrm{~mm}$ and $200 \mathrm{~mm} \times 200 \mathrm{~mm}$, respectively. The spectra show a reduction of the proton events just below the proton edge with increasing depth. At depth, the beam is hardened and the average proton energy increases which results in a shift to lower proton event sizes. At all investigated field sizes, $\epsilon^{*}$ decreases by 6-7 \% at $150 \mathrm{~mm}$ depth as compared to $50 \mathrm{~mm}$ depth. For all depths, variations in $R$ are more pronounced.

Variations with field size: Figures 20, 21, 22 show A-150 spectra measured at depths of $50 \mathrm{~mm}, 100 \mathrm{~mm}$ and $150 \mathrm{~mm}$, respectively. For all investigated depths, changes in field size result in slight variations in the microdosimetric spectra. For a constant depth an increase in the field size from $2500 \mathrm{~mm}^{2}$ to $40,000 \mathrm{~mm}^{2}$ results in an increase of $\epsilon^{*}$ of 2-4 \%. Again, changes in $R$ are more pronounced. Changes 


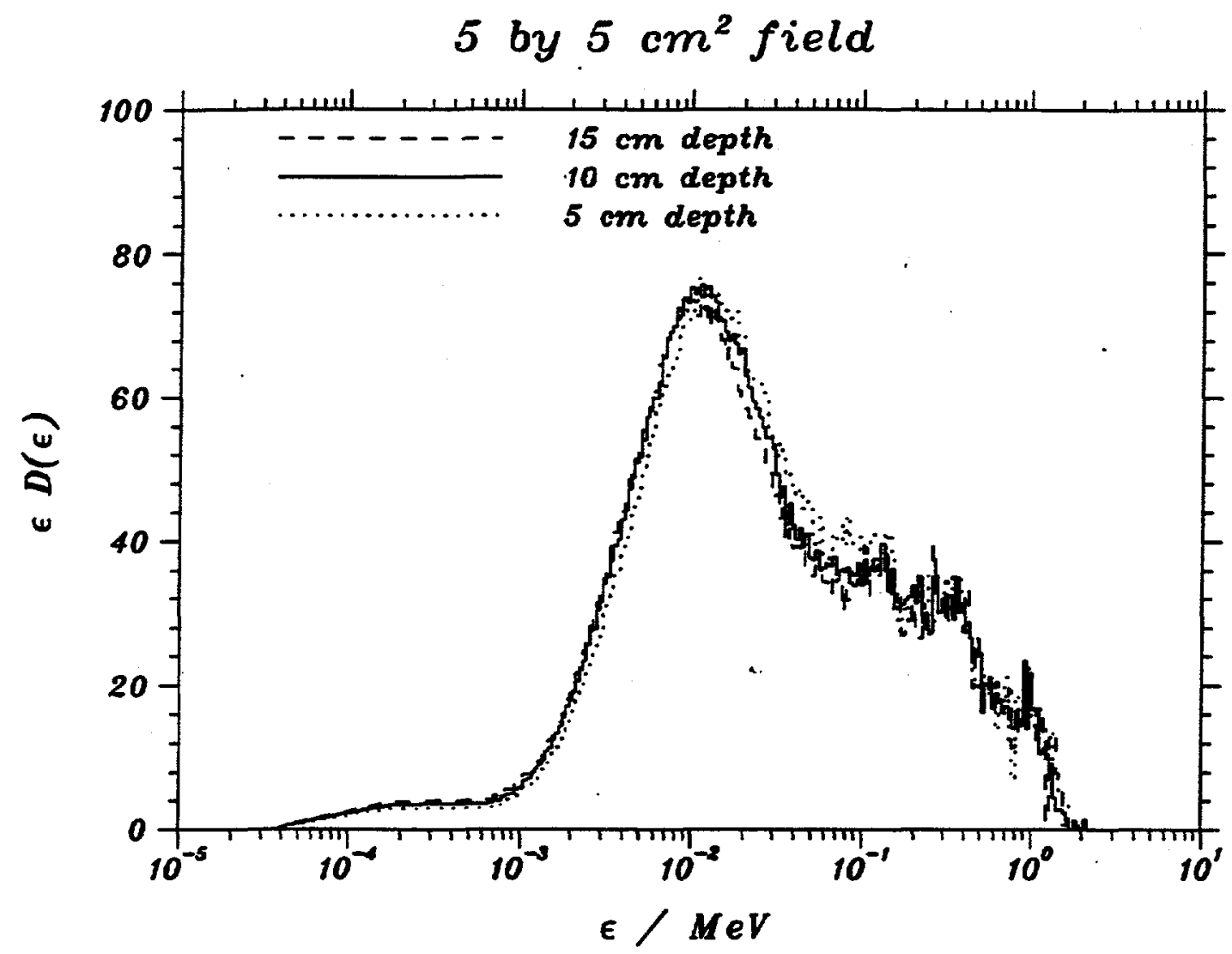

Figure 17: Microdosimetric spectra measured at a field size of $50 \mathrm{~mm} \times 50 \mathrm{~mm}$. 


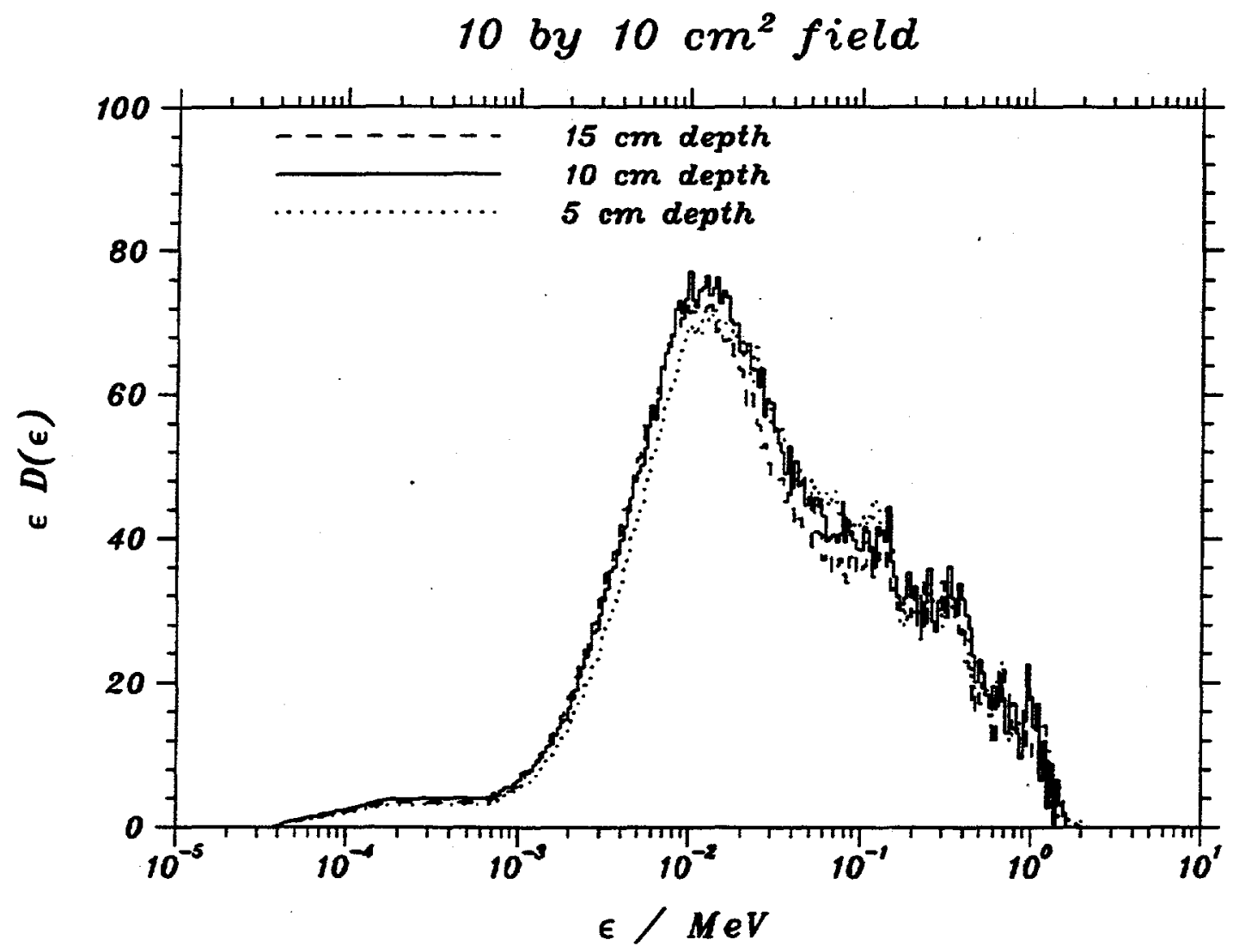

Figure 18: Microdosimetric spectra measured at a field size of $100 \mathrm{~mm} \times 100 \mathrm{~mm}$. 


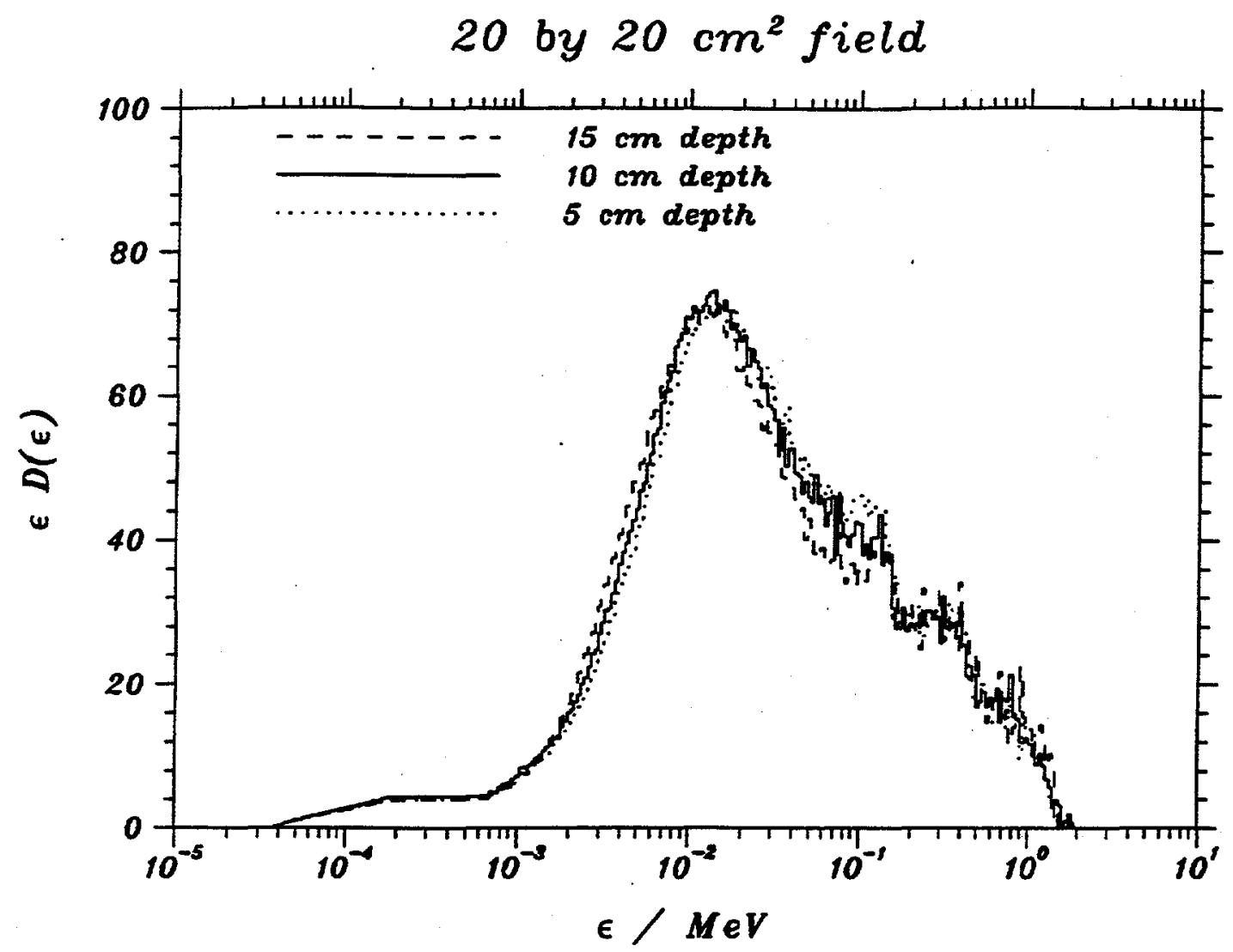

Figure 19: Microdosimetric spectra measured at a field size of $200 \mathrm{~mm} \times 200 \mathrm{~mm}$. 


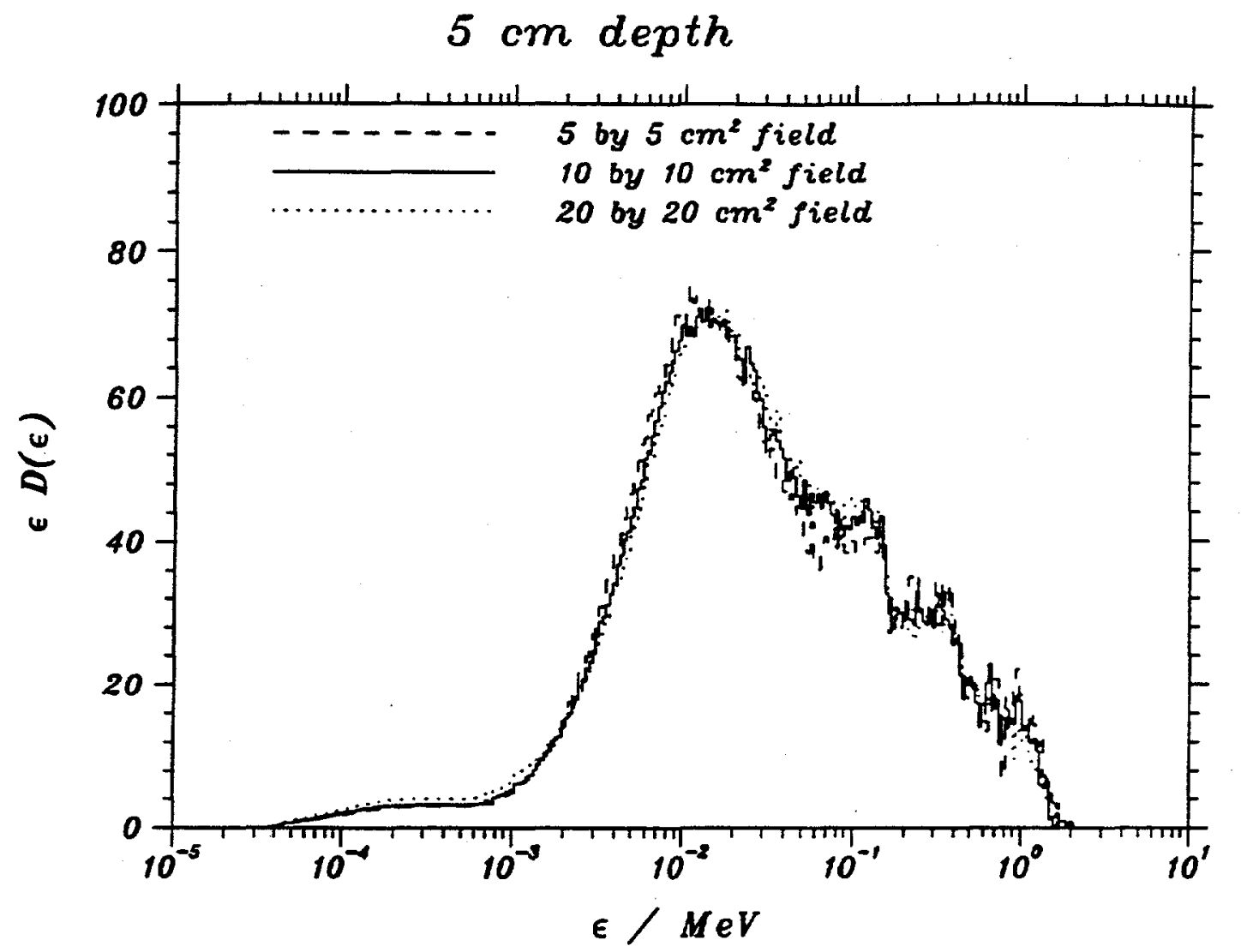

Figure 20: Microdosimetric spectra measured at a depth of $50 \mathrm{~mm}$.

in field size result in smaller variations in $\epsilon^{*}, R$ and the microdosimetric spectra, than comparable changes with depth.

Maximal variations: Figure 23 shows spectra measured at $150 \mathrm{~mm}$ depth in a $50 \mathrm{~mm} \times 50 \mathrm{~mm}$ field and $50 \mathrm{~mm}$ depth in a $200 \mathrm{~mm} \times 200 \mathrm{~mm}$ field. The first corresponds to the hardest and the latter to softest spectrum measured. The difference in shape of these two spectra is clearly seen and is quantified by a decrease of $\epsilon^{*}$ by $9 \%$ and $R$ by $15 \%$, moving from the softest to hardest spectrum. 


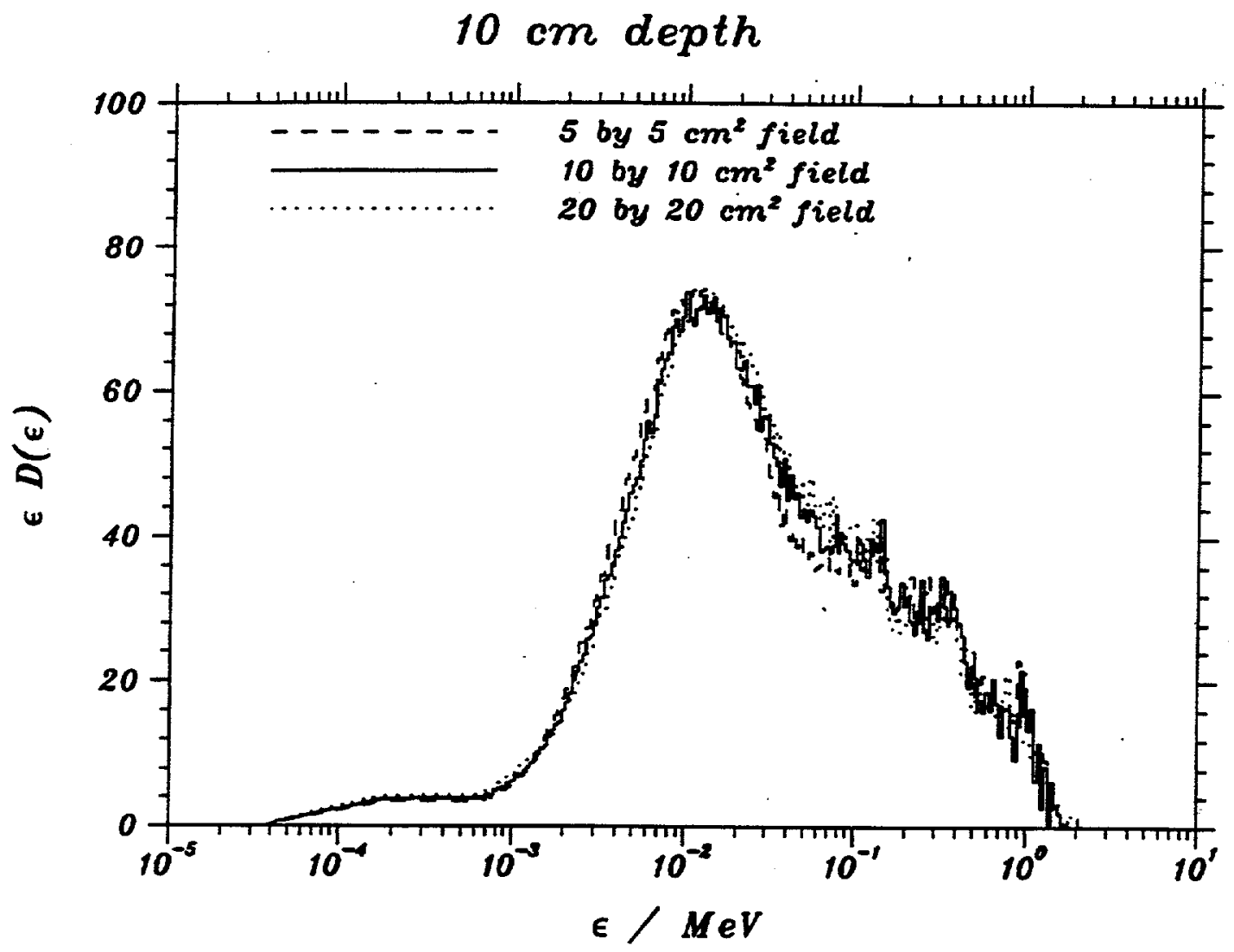

Figure 21: Microdosimetric spectra measured at a depth of $100 \mathrm{~mm}$. 


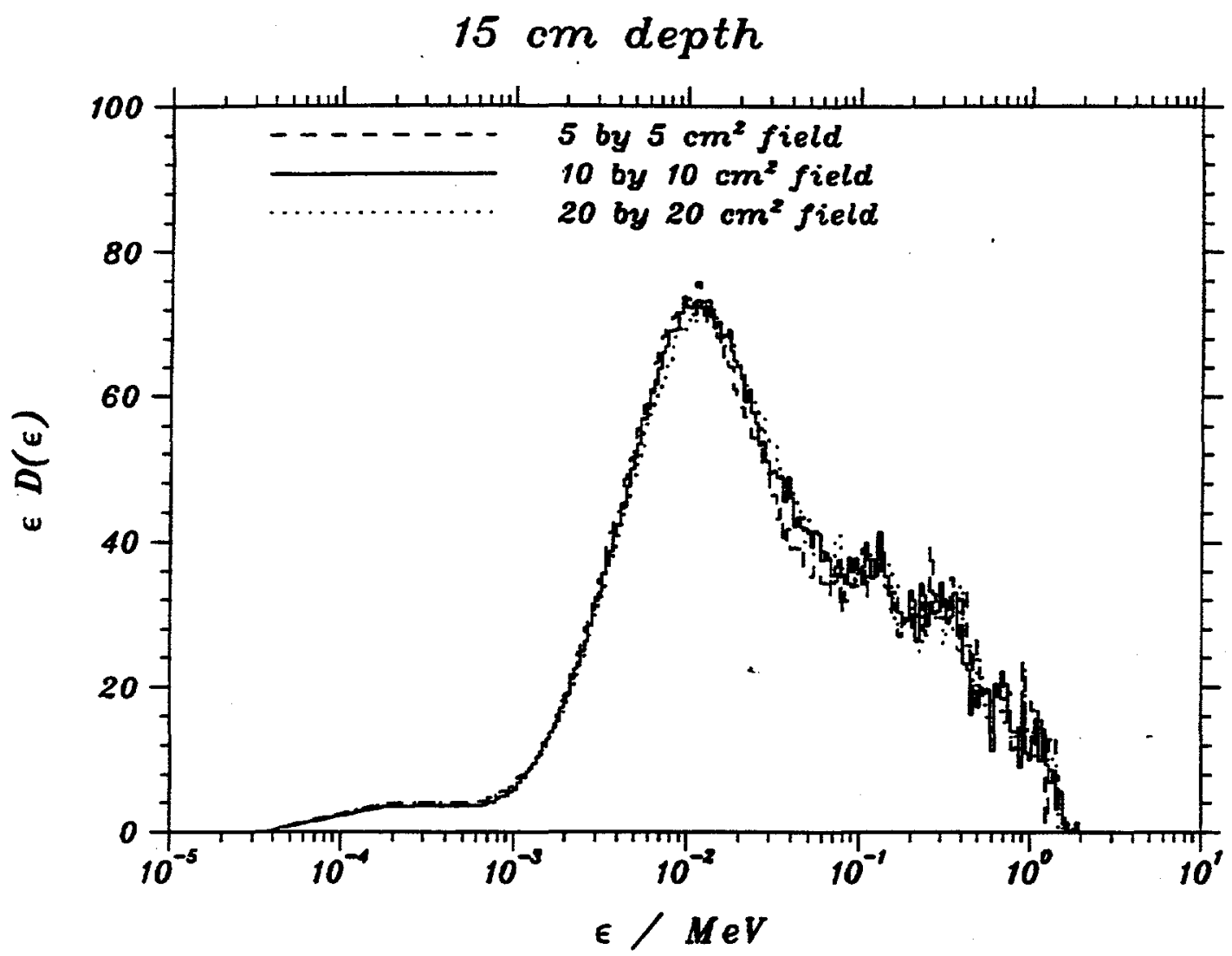

Figure 22: Microdosimetric spectra measured at a depth of $150 \mathrm{~mm}$. 


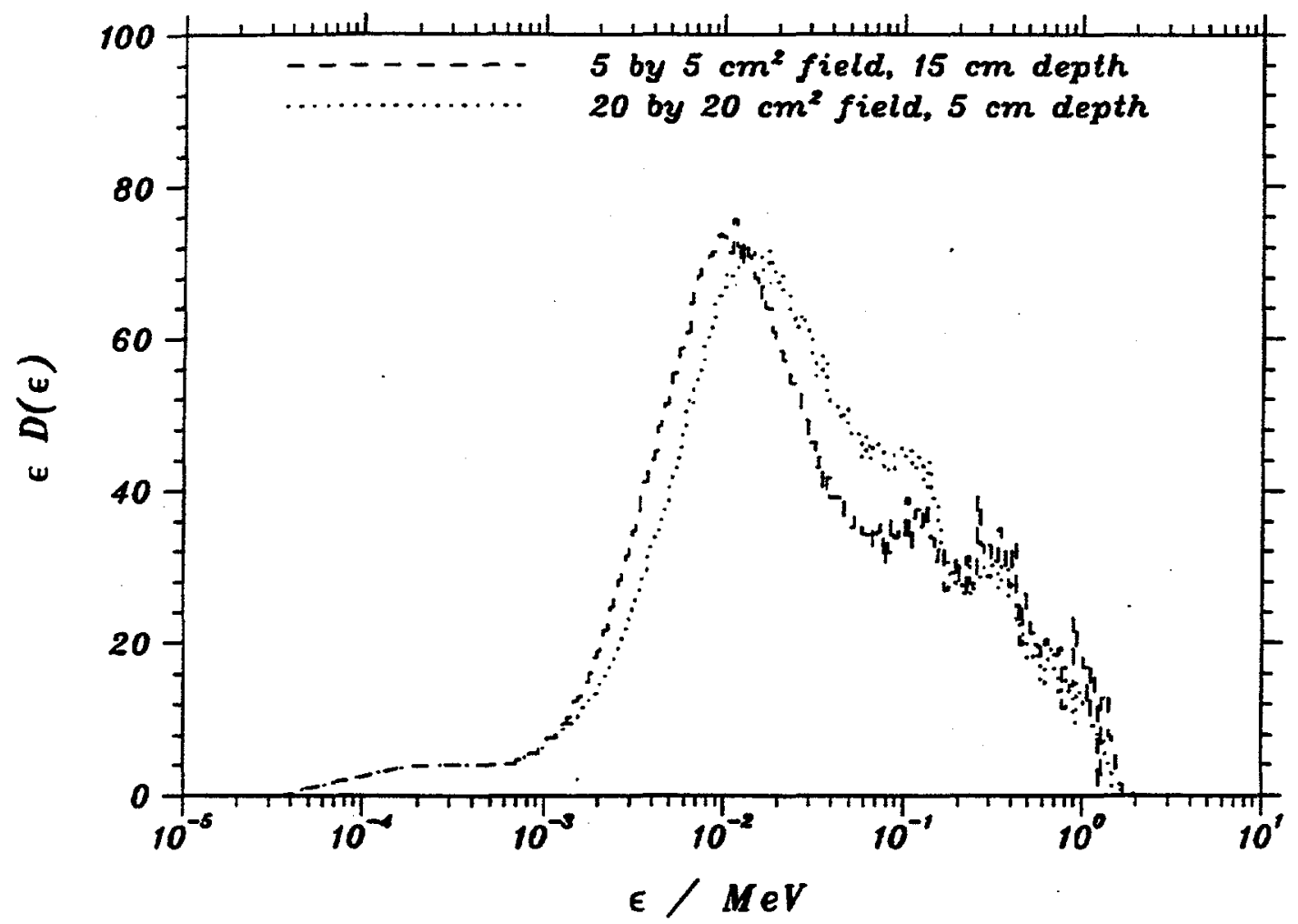

Figure 23: Spectra measured at $150 \mathrm{~mm}$ depth in a $50 \mathrm{~mm} \times 50 \mathrm{~mm}$ field and $50 \mathrm{~mm}$ depth in a $200 \mathrm{~mm} \times 200 \mathrm{~mm}$ field. 


\subsection{Discussion}

Besides mapping $\epsilon^{*}$ and $R$ variations in the NTF beam, the absolute $\mathrm{R}$-value for the normalization conditions was calculated. A R-value of $1.08 \pm 0.04$ suggests that the Fermilab beam is somewhat softer than the Louvain-la-Neuve beam. The proton energy at both facilities is essentially the same, however, the Louvain beam is, in fact, produced in a thinner beryllium target $(17 \mathrm{~mm})$ and the neutron beam is filtered by $20 \mathrm{~mm}$ polyethylene and $3 \mathrm{~mm}$ lead [38]. Each of these difference leads to a harder beam as compared to the NTF beam.

Variations in $\epsilon^{*}$ with depth can be directly compared to similar studies done at NAC in South Africa due to the similarity of the two facilities. In the unfiltered beam at NAC a reduction in $y^{*}$ of $6 \%$ was measured in a $200 \mathrm{~mm} \times 200 \mathrm{~mm}$ field between $25 \mathrm{~mm}$ and $150 \mathrm{~mm}$ depth [52]. At NTF a decrease in $\epsilon^{*}$ of $7 \%$ was measured between $50 \mathrm{~mm}$ and $150 \mathrm{~mm}$ depth. These results confirm the similarity of the two neutron beams.

Variations in $\epsilon^{*}$ due to changes in field size in the NTF beam also compare well to similar investigation in the NAC beam [6]. At NAC a 3-5\% variation in $y^{*}$ was found whereas at NTF a 2-4\% variation in $\epsilon^{*}$ was found for similar changes in field sizes.

Hill et al. [19] performed radiobiological experiments in the NTF beam and measured a 12-15\% reduction in RBE between $30 \mathrm{~mm}$ and $240 \mathrm{~mm}$. Assuming a uniform rate of beam hardening, Hill's results agree with the decrease of $\epsilon^{*}$ found in this study. However, Hill et al. [19] did not find any variations in RBE with collimator size.

Variations in $\epsilon^{*}$ compare well to predicted variations in $\mathrm{RBE}$ based on previous studies, and can therefore be used as a reliable tool to study beam quality changes. The calculation of RBE relative to other facilities using Pihet's $r(\epsilon)$-function agrees qualitatively with predictions. Calculations of $R$ variations are, in general, more 
pronounced than $\epsilon^{*}$ variations. However, they qualitatively agree with calculated $\epsilon^{*}$ variations.

Maximal variations between irradiation conditions result in $\epsilon^{*}$ and $R$ variations of up to 9 and $15 \%$ respectively. These variations introduce large errors in the calculation of absorbed dose, compared to the proposed accuracy in neutron therapy of $3.5 \%$ [31].

Many neutron therapy facilities use a polyethylene filter to reduce RBE variations with depth. The implementation of a $2.5 \mathrm{~mm}$ polyethylene filter at NAC reduced the variations in $y^{*}$ to $5 \%$ between $25 \mathrm{~mm}$ and $200 \mathrm{~mm}$ [52]. The remaining variations with depth are included in the treatment planning system at NAC [6].

In a separate study Rosenberg et al. [44] determined that the attenuation of the NTF beam by $25 \mathrm{~mm}$ polyethylene filter is only about $20 \%$.

The implementation of $a$ hardening filter or the inclusion of $R B E$ variations with field size and depth in the treatment planning system at NTF, should be considered. 


\section{Chapter 7}

\section{Boron Neutron Capture}

\subsection{Introduction}

Boron neutron capture therapy (BNCT) aims at conforming the absorbed dose to the tumor tissue by selectively loading the tumor tissue with a boron-10 compound and subsequently irradiating the tumor with thermal neutrons: Boron-10 has a high cross section (3830 barn) for thermal neutron capture and then branches into the two following reactions. With a $6 \%$ branching ratio a ${ }^{4} \mathrm{He}$ and a ${ }^{7} \mathrm{Li}$ particle are produced which share $2.8 \mathrm{MeV}$ of kinetic energy. With a $94 \%$ probability a ${ }^{4} \mathrm{He}, \mathrm{a}{ }^{7} \mathrm{Li}$ and a $0.48 \mathrm{MeV}$ photon are produced which leaves a kinetic energy of $2.3 \mathrm{MeV}$ to be shared between the two charged particles. The cross section of this capture reaction has a $1 / v$ dependence, where $v$ is the neutron speed. The ${ }^{4} \mathrm{He}$ and ${ }^{7} \mathrm{Li}$ particles have ranges in water of approximately $10 \mu \mathrm{m}$ and $5 \mu \mathrm{m}$, respectively. Due to these short ranges the absorbed dose is confined to the tumor tissue.

BNCT was proposed by Locher in 1936 [27] and first clinical trials were launched in the early fifties using reactor neutron beams. Results were unacceptable due to severe normal tissue damage which was caused by the presence of boron in normal tissue. After improved boron compounds were developed, the interest in BNCT arose again during the mid-eighties. There are currently two facilities (Brookhaven National Laboratory and Massachusetts Institute of Technology) in the US that have started to use reactor beams for BNCT trials on brain tumors and melanomas [14]. 
Reactor beams exhibit limited penetration and Waterman et al. [61] first suggested the utilization of low energy neutrons produced by the slowing down of higher energy neutrons in fast neutron beams. Applying this concept, the feasibility of using BNC in the fast neutron therapy beam at Fermilab in order to boost the tumor dose was investigated. If BNCT is the sole form of treatment, one needs to ensure that each tumor cell experiences a boron neutron capture reaction, which necessitates the loading of every cell with boron-10. In addition, the boron loading of normal tissue needs to be significantly lower. This sets high demands on the tumor specificity of the boron compound. On the other hand, if boron neutron capture could be used just for a tumor dose enhancement the above requirement would no longer be as stringent.

\subsection{Clinical rationale}

The expected survival time for patients diagnosed with glioblastoma multiforme, a type of brain tumor, is around eight months following diagnosis and can be extended by another four months with radiation therapy. There is no difference in survival between fast neutron and photon patients but the cause of death has been found to vary with treatment modality. A regrowth of the tumor is the primary cause of death in photon patients whereas with fast neutron therapy one achieves tumor control but patients sustain a high degree of normal tissue damage that causes death [48]. These clinical results indicate a small therapeutic ratio of glioma to normal brain tissue. To increase the survival, the therapeutic ratio needs to be increased. BNCT has the potential of achieving just that in neutron beams. A selective dose enhancement in the tumor tissue will allow a corresponding reduction in the overall absorbed dose level, therefore reducing the normal tissue complications. 


\subsection{BNC in fast neutron beams}

The enhancement due to boron neutron capture in fast neutron therapy beams has been investigated by several groups for a variety of therapy beams [61, 62, 42, 29]. Waterman et al. [61] studied the dose enhancement due to B-10 in four neutron beams which varied in median energy from 2.4 to $9 \mathrm{MeV}$. The thermal neutron fluence was determined with a $B F_{3}$ proportional counter and Va-23 activation. The absolute dose enhancement due to boron was between $3.5 \%$ and $16 \%$ for a typical boron loading of $50 \mathrm{ppm}$.

More recently Pöller et al. [42] used Monte Carlo techniques to deduce the dose enhancement due to neutrons in a $\mathrm{d}(14) \mathrm{Be}$ beam used at Essen. Gold foil activation measurements were done to verify the Monte Carlo calculations. Results were in agreement with Waterman [61] and calculations were in reasonable agreement with gold foil measurements. A $7.5 \%$ absorbed dose enhancement was found at $60 \mathrm{~mm}$ depth and for a $50 \mathrm{ppm}$ boron loading.

Wootton et al. [62] investigated several neutron beams produced by protons and deuterons of varying energy on beryllium targets of different thicknesses by means of sodium activation. In addition to that, several beams were filtered with iron and perspex filters. For a $\mathrm{p}(50.5) \mathrm{Be}(26)$ beam a boron dose enhancement of $3 \%$ was deduced for a $50 \mathrm{ppm}$ loading.

At the p(48.5)Be neutron therapy facility in Detroit, Kota [22] measured a dose enhancement of $2.5-3.5 \%$ for a $50 \mathrm{ppm}$ boron-10 loading.

\subsection{Beam Modifications}

The dose enhancement due to boron is proportional to the boron concentration in tumor tissue and to the thermal and epithermal neutron fluence. We investigated an enhancement of the latter quantity. The effect of various filter materials on the neutron energy spectrum was simulated using Monte Carlo codes. Results were 
used to select promising filter materials that were then investigated in the actual beam. The influence of the collimator material was tested in the actual beam. Additionally, the primary neutron energy spectrum can be changed by changing the proton beam energy.

Monte Carlo Calculations. Calculations were performed using MCNP [8], a Monte Carlo code from Los Alamos. MCNP uses data files that normally extend up to $20 \mathrm{MeV}$. Extended data libraries up to $100 \mathrm{MeV}$ for use by MCNP exist for some selected elements [64] and were used after they became available to us. The NTF neutron spectrum recently calculated by Ross et al. [45] was used as the source spectrum for the Monte Carlos calculations. The simulated geometry is shown in figure 24. Neutrons are emitted into a cone to simulate a collimated neutron beam. At isocenter we simulated a head phantom that is surrounded with a $50 \mathrm{~mm}$ thick bone surface and filled with water. Filters were placed adjacent to the head phantom to minimize loss of thermal neutrons produced in the filter due to the $1 / r^{2}$ effect.

Collimator and Filter Materials. Besides determining the influence of filters on the neutron beam, changes introduced by different collimator materials were investigated. Neutron scattering of the collimator material contributes to the lower energy component of the beam. At NTF, the regular collimator is a mixture of concrete and polyethylene pellets. The influence of steel blocks was investigated. Proton Beam Modifications. At NTF, $66 \mathrm{MeV}$ protons from a linear accelerator (linac) are used to generate the neutron beam. As described in section 2.3, the linac is divided into several tanks and protons designated for NTF are only accelerated in tanks one through three. Protons exit tank three with an energy of $66 \mathrm{MeV}$ and drift through tank four to where NTF is located. Protons enter tank three with an energy of $37 \mathrm{MeV}$. It is possible to turn tank three off such that protons drift through tank three and four before they are diverted into the NTF beam line. The proton energy can in this way be reduced to $37 \mathrm{MeV}$. The 


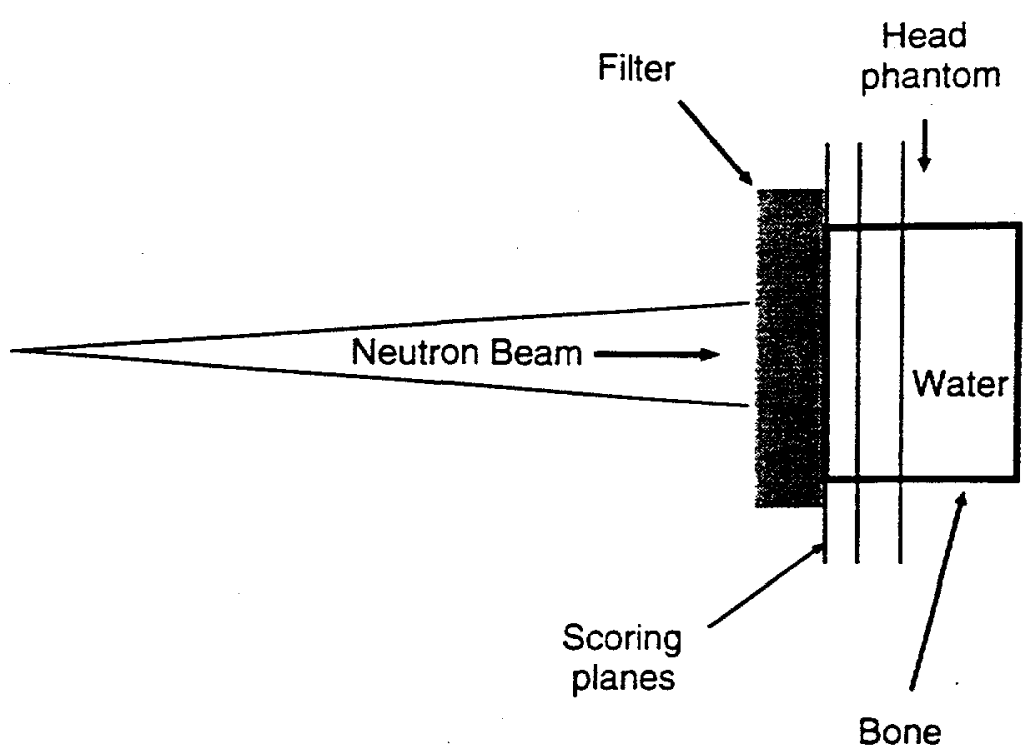

Figure 24: Geometry used for Monte Carlo calculations.

resulting neutron beam is of lower energy and should also contain a lower thermal and epithermal component since $37 \mathrm{MeV}$ protons are completely stopped in the beryllium target.

\subsection{Measurements}

In principal one can measure the thermal neutron fluence at the tumor location and calculate the dose enhancement due to B-10. Alternatively, the boron dose enhancement can be determined by exposing a boron-loaded and a regular A-150 plastic counter to the same neutron fluence. Walls of the loaded counter are made out of A-150 with an additive of 200 ppm B-10. For this purpose boron nitrate with an average particle size of $1 \mu \mathrm{m}$ was mixed into the $\mathrm{A}-150$ plastic. The regular counter is identical to the boron loaded counter in every aspect except the boron loading of the wall. A difference in the measured absorbed dose is then due to the B-10 loading. 


\subsection{Results}

For all measurements two counters, the regular A-150 counter and the B-10 loaded A-150 counter, were irradiated simultaneously in the neutron field. Unless otherwise stated, a $100 \mathrm{~mm} \times 100 \mathrm{~mm}$ field was used. A head shaped lucite phantom was filled with water and counters were immersed in the phantom at a approximate water depth of $50 \mathrm{~mm}$. For this to be feasible, the head phantom was turned upside down.

The uncertainty in the dose ratio of the two A-150 counters is estimated to be $4 \%$ due to a $3 \%$ counting statistics uncertainty in both spectra.

The boron-loaded counter is filled with regular isobutane tissue equivalent gas and the lack of a boron-loading of the gas needs to be considered. Since both particles that are generated in the B-10 reaction have ranges in tissue that are comparable to the counter volume and may therefore not be able to cross the counter cavity, the measured boron dose enhancement in the counter underestimates the true boron dose enhancement in a homogeneous tumor. Wuu et al [63] calculated that, for a $2 \mu \mathrm{m}$ gas filling, $75 \%$ of the dose is due to particles crossing the cavity. If $25 \%$ of the dose is due to particles stopping in the gas cavity, it can be estimated that, if stoppers are only depositing about half of the energy that crossers do, about $13 \%$ of the gas dose is lost due to the lack of boron-10 in the gas. To eliminate this effect, the boron-loaded counter can be filled with tissue equivalent gas that contains an additive of boron trifluoride such that the gas is loaded with the same amount of B-10 as the wall.

Enhancement in the open beam. Firstly, the boron dose enhancement in the open field was determined. In anticipation of the filter investigations, in which the dose rate is decreased, the counters were positioned about $300 \mathrm{~mm}$ closer to the target than the isocenter for all measurements. Figure 25 illustrates the setup.

The result of this measurement is shown in figure 26 . A $3 \% \pm 4 \%$ boron dose 


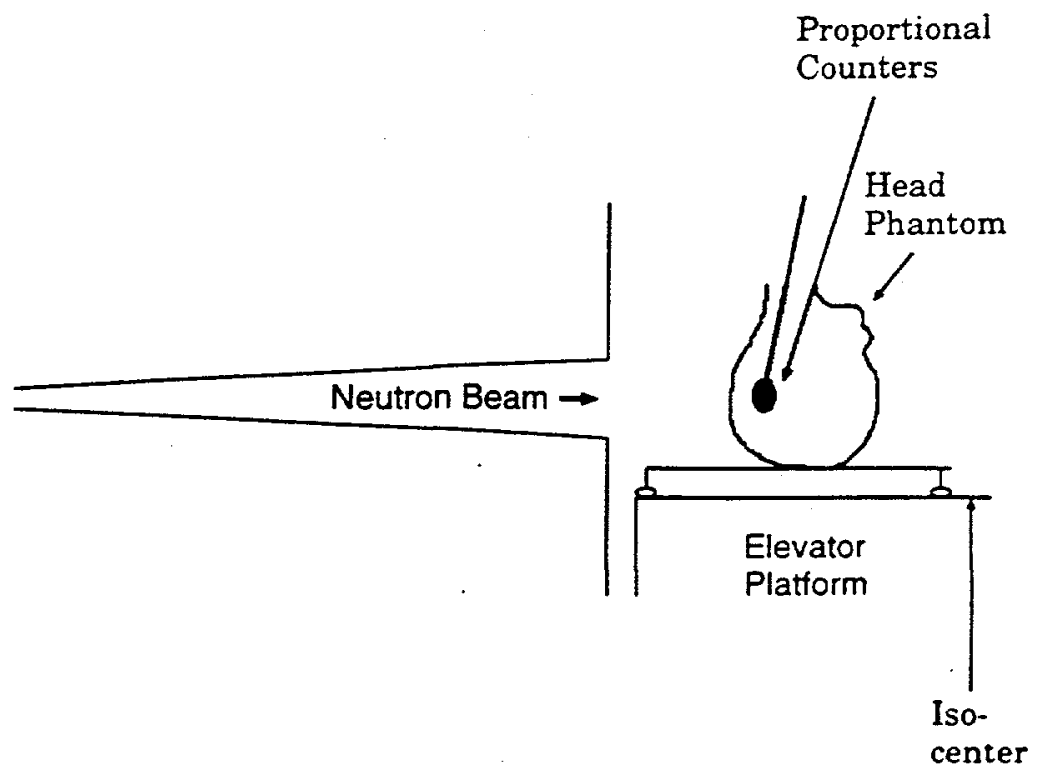

Figure 25: Side view of counter setup in the treatment room for the open field $\mathrm{BNC}$ measurements.

enhancement was measured. Considering that the B-10 counter is loaded with $200 \mathrm{ppm}$ and a typical tumor loading of only $50 \mathrm{ppm}$, this measurement shows a negligible small enhancement.

Monte Carlo Calculations. Based on initial Monte Carlo calculations and the work done at the Harper Grace neutron therapy facility in Detroit [22], we focused on the investigation of tungsten as a filter material. Calculations on the effect of filter thickness showed that the increase in the low energy component relative to the high energy component, increases with increasing filter thickness. The calculated spectra are shown in Figure 27. Spectra are normalized to the highest energy bin. Enhancement in the modified beam. To measure the effect of tungsten filtration, a $90 \mathrm{~mm}$ tungsten filter was placed in the neutron beam. Figure 28 illustrated the setup. A $90 \mathrm{~mm}$ tungsten filter was used because this was all the tungsten that was easily available at NTF. Results of this measurement are shown in figure 29 . 


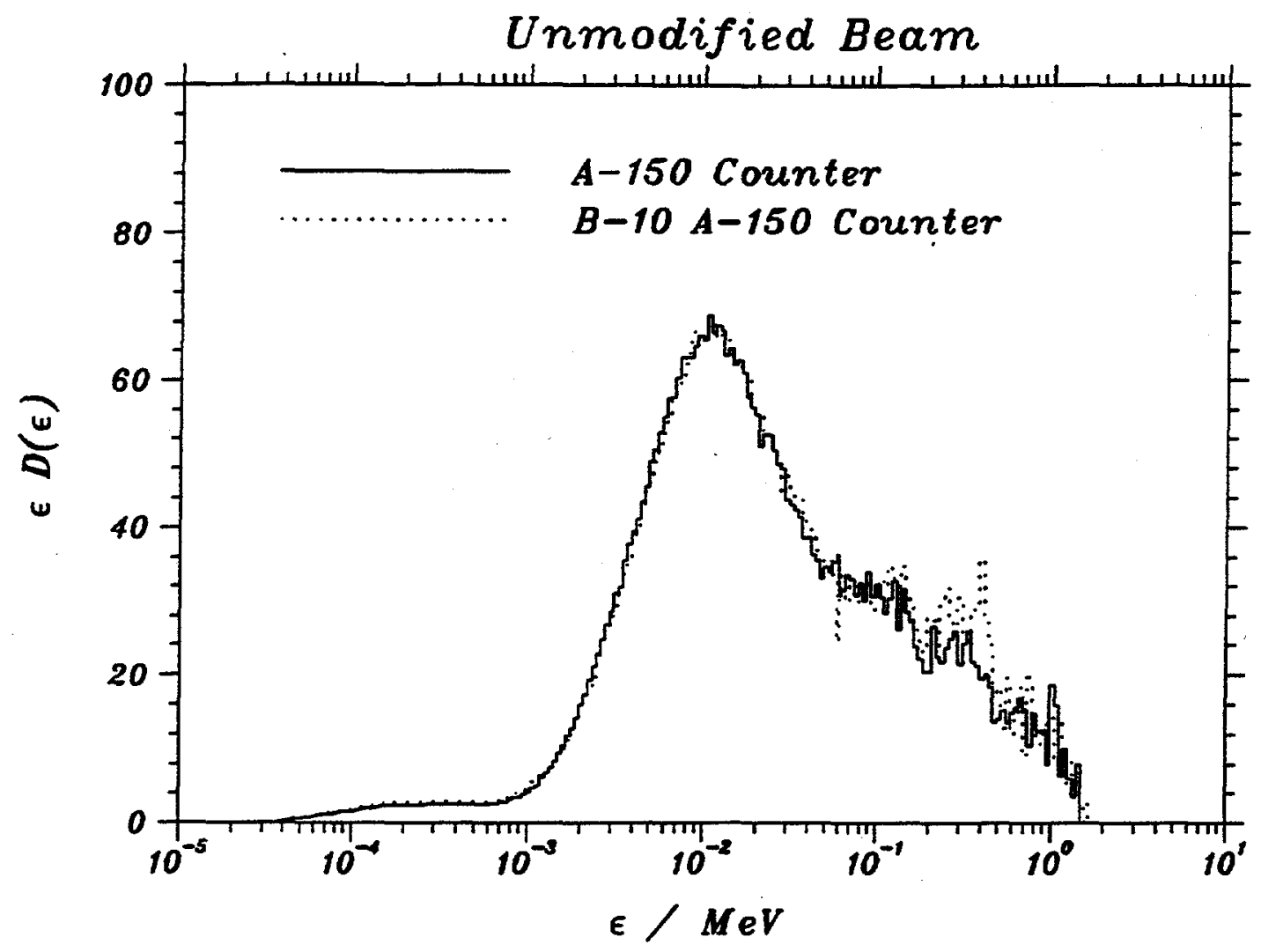

Figure 26: Microdosimetric spectra measured in the unmodified beam. 


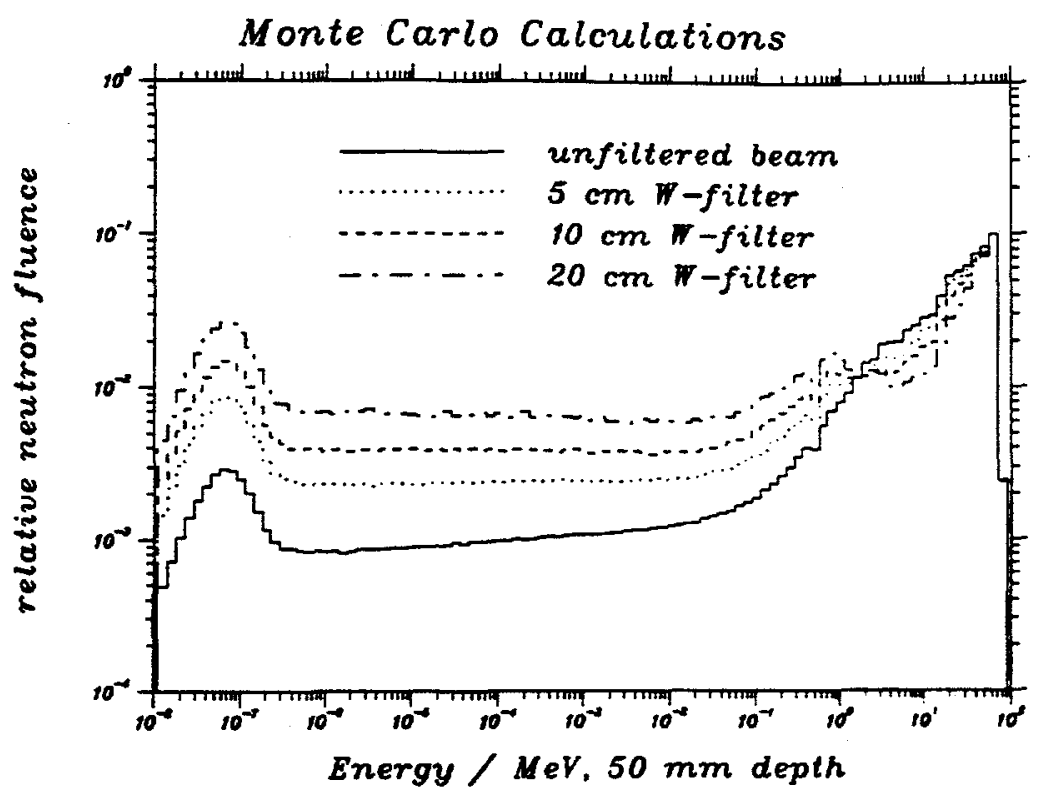

Figure 27: Results of MCNP calculations on the effect of tungsten filtration of the NTF beam.

The measured boron dose enhancement was boosted to $5 \%=4 \%$ as compared with the unmodified beam. This enhancement is, however, still insufficient.

To test the influence of the collimator material on the neutron spectrum, a $240 \mathrm{~mm} \times 240 \mathrm{~mm}$ size collimator was blocked down to a $120 \mathrm{~mm} \times 120 \mathrm{~mm}$ field size. For this, steel blocks, that extend $200 \mathrm{~mm}$ into the collimator, were used. Figure 30 illustrates this setup. Results are shown in figure 31. Again, the measured boron dose enhancement was slightly boosted to $5 \% \pm 4 \%$ as compared with the unmodified beam.

If both, the $90 \mathrm{~mm}$ tungsten filter and steel blocks, are used, the boron dose enhancement is boosted to $7 \% \pm 4 \%$. Results for this run are shown in figure 32 .

Last, the reduction of the proton energy to $37 \mathrm{MeV}$ was investigated. In addition to decreasing the proton energy, the resulting neutron beam was also filtered by $90 \mathrm{~mm}$ tungsten. Results are shown in figure 33. With this setup the boron dose enhancement increases to $12 \% \pm 4 \%$. 


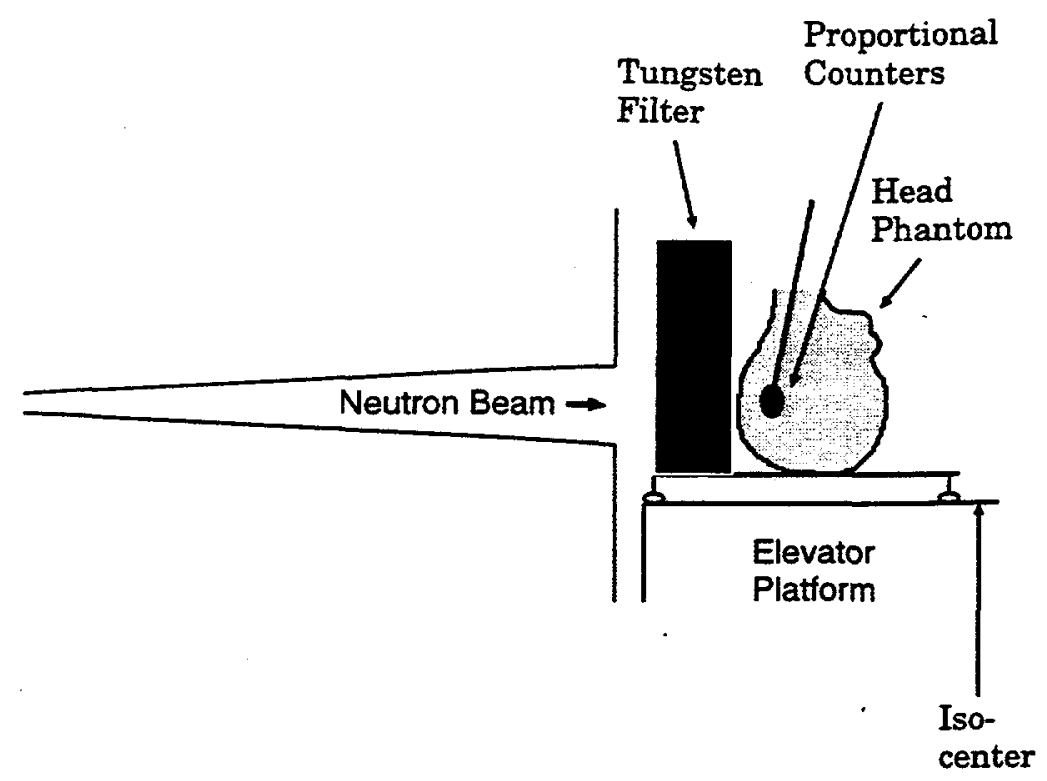

Figure 28: Side view of counter setup in the treatment room for the tungsten filtered beam BNC measurements.

\subsection{Discussion}

The above measurements show that at most a $3 \%$ boron dose enhancement can be achieved in the modified neutron beam for a $50 \mathrm{ppm}$ boron loading.

Even though, it is possible to further filter the beam, e.g., to use a thicker tungsten filter, there are practical limitations to this. Using a $90 \mathrm{~mm}$ tungsten filter reduces the dose rate to $20 \%$, which would result in treatment time up to 30 minutes. Much longer treatment times are unacceptable. The dose rate reduction due to the proton energy change is not known, but this further reduction might already be unacceptable. It also has to be kept in mind that the modified beam has a different RBE. Figure 34 shows the A-150 spectra measured in modified beam and the regular beam. Convolution of both spectra with a biological weighting function indicates a $20 \%$ increase in the modified beam's RBE, which indicates that a modified beam may have quite different characteristics which need to be 


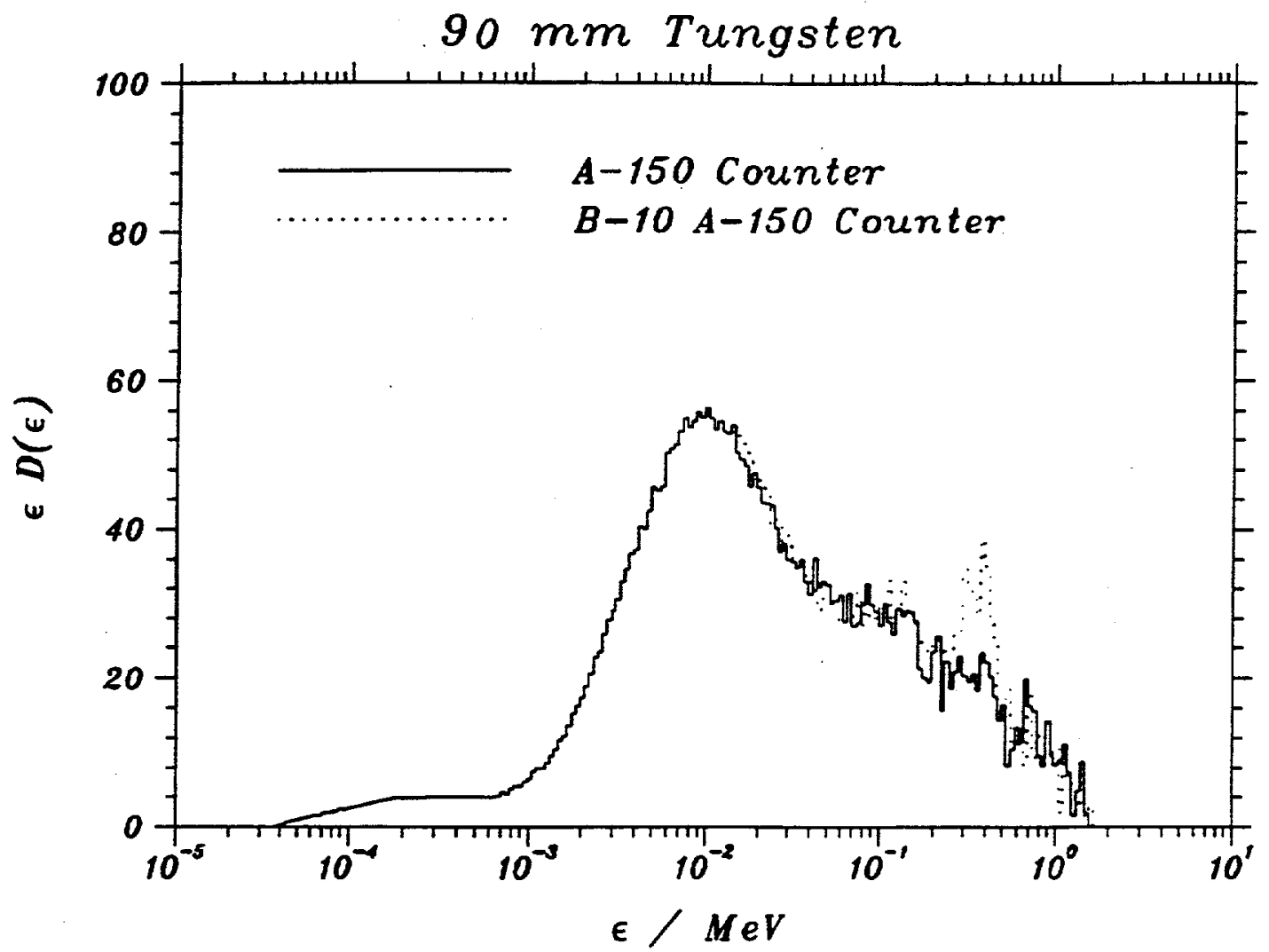

Figure 29: Microdosimetric spectra measured in the tungsten filtered beam. 


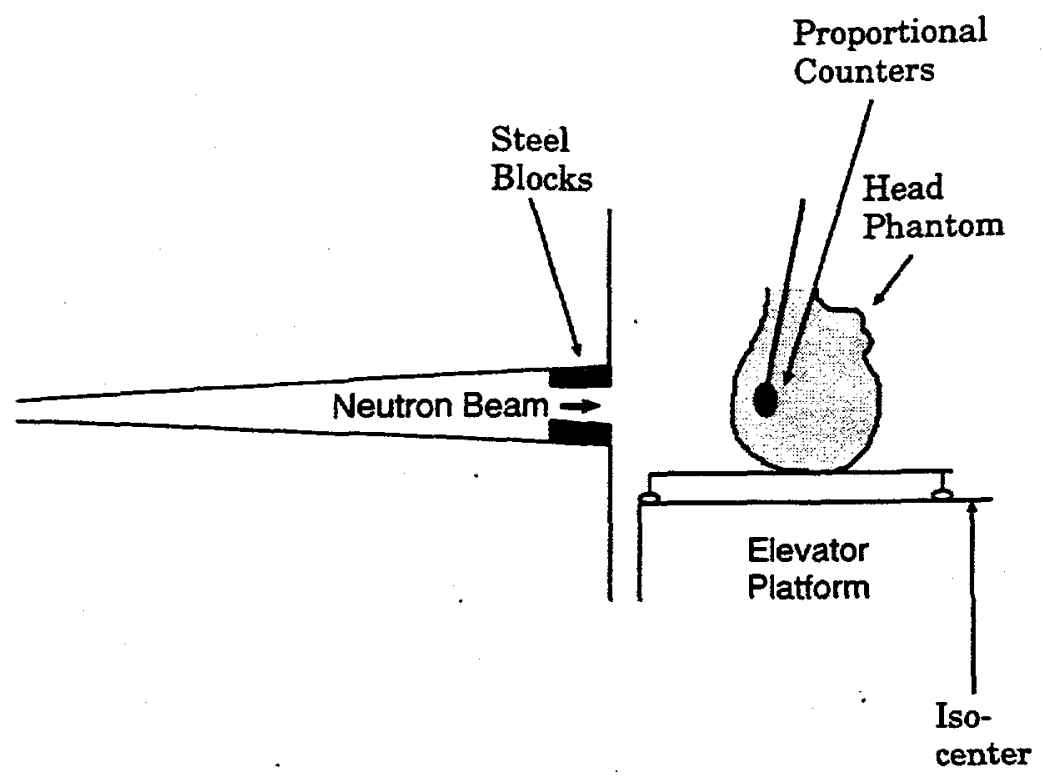

Figure 30: Side view of counter setup in the treatment room for the steel collimated BNC measurements.

taken into account.

In conclusion, the use of BNC in the fast neutron beam is not feasible, unless the neutron spectra is further modified or, better, boron compounds that achieve a higher boron concentration in the tumor tissue become available. 


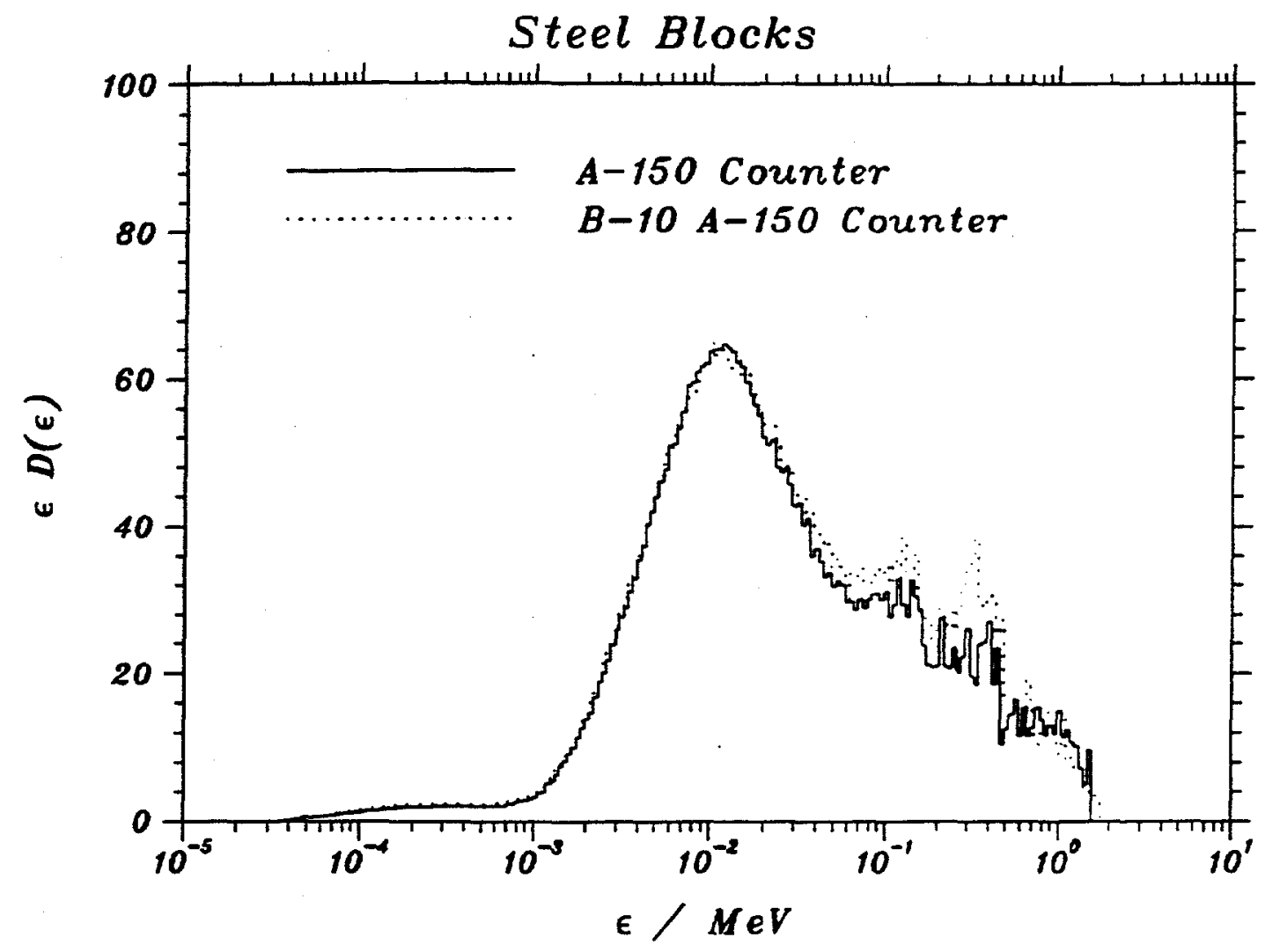

Figure 31: Microdosimetric spectra measured in the steel block collimated field. 


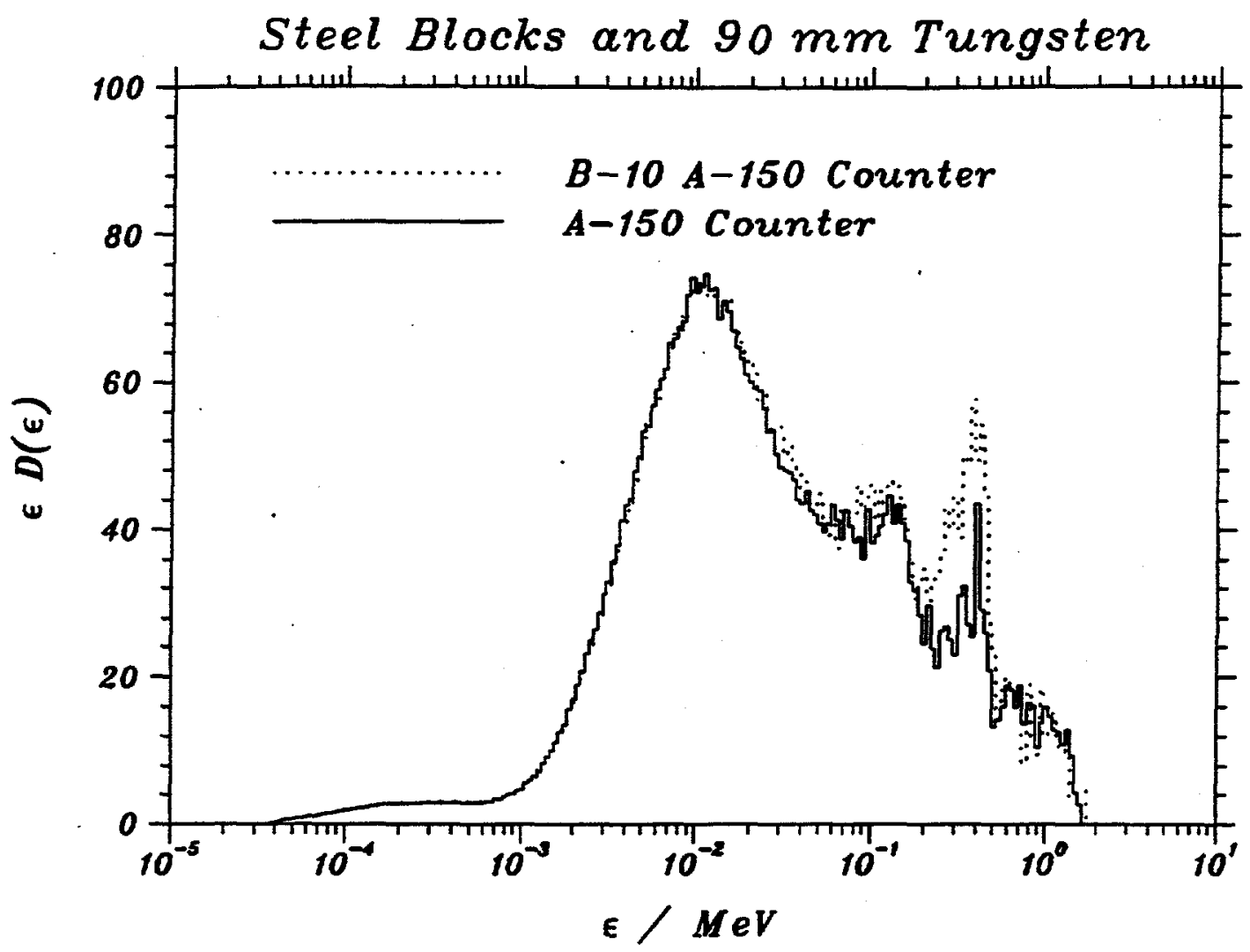

Figure 32: Microdosimetric spectra measured in the steel block collimated and tungsten filtered field. 


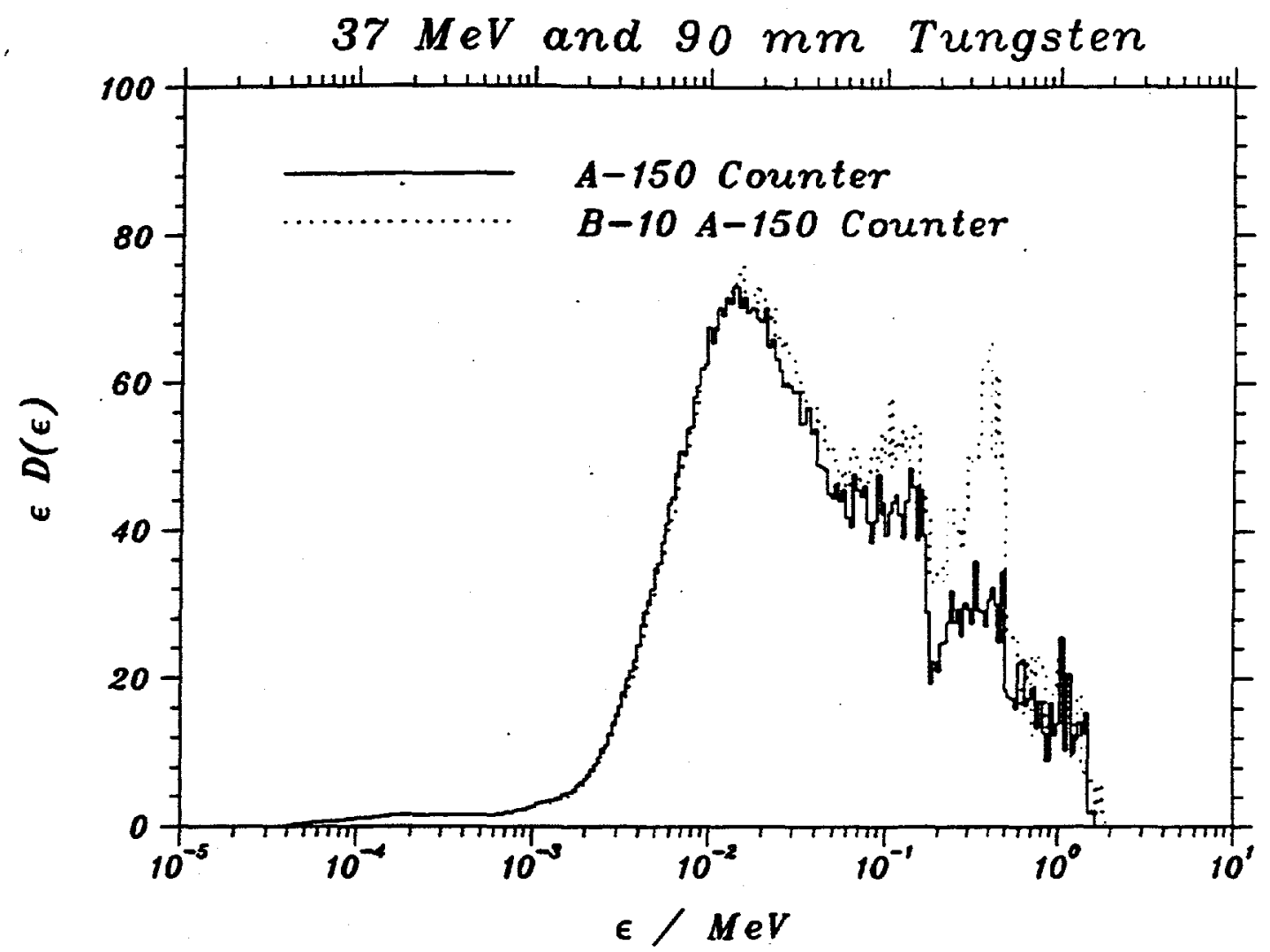

Figure 33: Microdosimetric spectra measured in the $37 \mathrm{MeV}$ and tungsten filtered beam. 


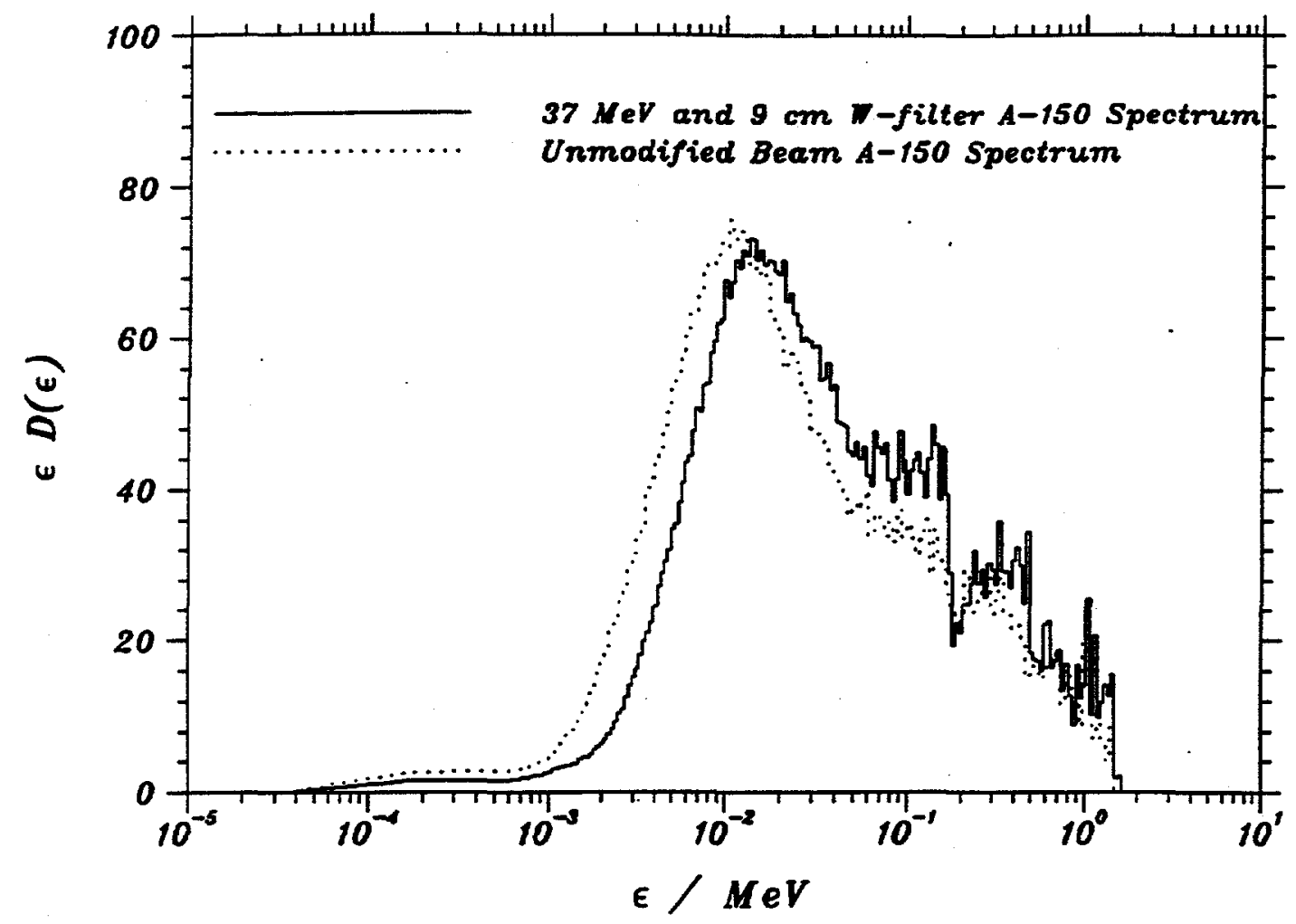

Figure 34: A-150 microdosimetric spectra measured in the modified and unmodified beam. 


\section{Chapter 8}

\section{Conclusions}

Using microdosimetry, the absorbed dose conversion factor from A-150 plastic to ICRU tissue was ascertained to be $0.92 \pm 0.04$. Currently, the recommended [34] conversion factor of 0.95 is used at NTF. This discrepancy leads to an overestimation of the NTF tissue dose by $3 \%$, i.e., NTF doses are $3 \%$ smaller than prescribed. Here a correct determination of the absorbed dose in A-150 tissue equivalent plastic is assumed. Due to the wealth of clinical information and experience obtained in this beam, its clinical effects are well characterized and optimized. An adjustment in the delivered doses is therefore not recommended. The overestimation in NTF doses is however particularly important when clinical results are compared between different neutron therapy facilities.

It would be well worth to repeat this investigation in other neutron therapy beams, since all facilities rely on recommended conversion factors. The determination of tissue dose is of paramount importance when clinical information is exchanged.

The qualitative information provided by microdosimetry was used to map variations in RBE related quantities throughout the beam. Variations were found to be significant and an incorporation of these into the treatment planning system, or, alternatively, the installation of a polyethylene filter in the NTF beam, should be considered.

Since there is such wealth of clinical information available for the NTF beam, a correlation of changes in $\epsilon^{*}$ and $\mathrm{R}$ with clinical effects could be investigated. Such a study would be very interesting and could potentially lead to a biological weighting 
function that could be used to predict variations in clinical effects based variations in the shape of microdosimetric spectra. NTF is probably the only facility that has accumulated enough clinical information for a such a study.

Results of our BNC study showed that in the regular NTF beam the boron dose enhancement for a typical boron concentration is negligible. The beam had to be modified significantly to achieve an enhancement of $3 \%$. In conclusion, we could not demonstrate the feasibility of using BNC to significantly enhance the tumor dose in the NTF beam.

The use of fast neutron beams in the treatment of glioma multiforme patients should still be investigated since neutron beams do control this tumor better than any other form of radiation therapy. An improvement in neutron therapy treatments for this particularly aggressive tumor relies on improvements in the tumor to normal tissue dose ratio. The implementation of stereotactic methods at neutron therapy facilities, would result in such an improvement. A study on the implementation of stereotactic radiation therapy at NTF would be very useful. 


\section{Bibliography}

[1] F. H. Attix. Introduction to Radiological Physics and Radiation Dosimetry. John Wiley and Sons, New York, 1986.

[2] M. Awschalom, I. Rosenberg, and A. Mravca. Kermas for various substances averaged over the energy spectra of fast neutron therapy beams: A study in uncertainties. Med. Phys., 10(4):395-409, 1983.

[3] G. W. Barendsen, H. M. D. Walter, J. F. Fowler, and D. K. Bewley. Effects of different ionizing radiations on human cells in tissue culture. Radiat. Res.,. 18:106-119, 1963.

[4] M. Beauduin, J. Gueulette, B. M. de Coster, V. Gregoire, M. Octave-Prignnot, S. Vynckier, and A. Wambersie. Radiobiological intercomparisons of fast neutron beams used in therapy. Strahlenther. Onkol., 165:263-267, 1989.

[5] D. K. Bewley. The physics and radiobiology of fast neutron beams. Adam Hilger, Bristol, England, 1989.

[6] P. J. Binns. Microdosimetry for a Fast Neutron Therapy Beam. PhD thesis, University of Cape Town, Cape Town, Republic of South Africa, June 1993.

[7] F. P. Brady and J. L. Romero. Neutron induced reactions in tissue resident elements, final report to the National Cancer Institute, grant no. 1R01 CA16261. Technical report, University of California-Davis, Davis, CA, 1979.

[8] J. F. Briesmeister (Editor). MCNP 4-A general Monte Carlo code for neutron transport and photon transport. Technical Report LA-12695-M, Los Alamos National Laboratory, 1993. 
[9] G. Bühler, H. Menzel, H. Schuhmacher, G. Dietze, and S. Guldbakke. Neutron kerma factors for magnesium and aluminum measured with low-pressure proportional counters. Phys. Med. Biol., 31(6):601-612, 1986.

[10] Bühler, G. Anwendung microdosimetrischer Methoden für die Dosimetrie und Messung von Ionisationsverteilungen mit metallischen Proportionalzählern für Neutronen zwischen $13.9 \mathrm{MeV}$ und $19 \mathrm{MeV}$. $\mathrm{PhD}$ thesis, Universität des Saarlandes, Saarbrücken, Federal Republic of Germany, February 1985.

[11] M. B. Chadwick, P. M. DeLuca, Jr., and R. C. Haight. Nuclear data needs for neutron therapy and radiation protection. Radiat. Prot. Dosim., 70:1-12, 1997.

[12] P. M. DeLuca, Jr., H. H. Barschall, Y. Sun, and R. C. Haight. Kerma factor of oxygen, aluminum, and silicon for $15-$ and $20-\mathrm{MeV}$ neutrons. Radiat. Prot. Dosm., 23:27-30, 1988.

[13] J. L. Fowler. Nuclear Particles in Cancer Treatment. Adam Hilger, Bristol, United Kingdom, 1981.

[14] R. Gahbauer, N. Gupta, T. Blue, J. Goodman, J. Grecula, A. H. Soloway, and A. Wambersie. BNCT: Status and dosimetry requirements. Radiat. Prot. Dosim., 70:547-554, 1997.

[15] T. W. Griffin, T. F. Pajak, G. E. Laramore, W. Duncan, M. P. Richter, F. R. Hendrickson, and M. H. Maor. Neutron vs photon irradiation of inoperable salivary gland tumors: Results of an RTOG-MRC Cooperative randomized study. Int. J. Radiation Oncology Biol. Phys., 15:1085-1090, 1988.

[16] E. Hall. Radiobiology for the Radiologist, Third Edition. J. B. Lippincott Company, Philadelphia, 1988. 
[17] E. J. Hall, M. Zaider, R. Bird, M. Astor, and W. Roberts. Radiobiological studies with therapeutic neutron beams generated by $\mathrm{p}+\mathrm{Be}$ or $\mathrm{d}+\mathrm{Be}$. Brit. J. Radiol., 55:640-644, 1982.

[18] C. L. Hartmann, P. M. DeLuca Jr., and D. W. Pearson. Measurement of neutron kerma factors in $\mathrm{C}, \mathrm{O}$, and $\mathrm{Si}$ at 18,23 , and $25 \mathrm{MeV}$. Radiat. Prot. Dos., 44(1/4):25-30, 1992.

[19] C. K. Hill, R. K. Ten Haken, and M. Awschalom. The lethal effects of Fermilab fast neutrons vary with the depth of cells in a water phantom. Int. J. Radiation Oncology Biol. Phys., 20:1341-1345, 1981.

[20] S. Hornsey, R. Myers, C. J. Parnell, S. W. Blake, and D. K. Bewley. Changes in relative biological effectiveness with depth of the Clatterbridge neutron therapy beam. Brit. J Radiol., 61:1058-1062, 1988.

[21] A. M. Kellerer and H. H. Rossi. The theory of dual radiation action. Current Topics in Radiation Research Quarterly, 8:85-158, 1972.

[22] C. Kota. Microdosimetric considerations in the use of the boron neutron capture reaction in radiation therapy. PhD thesis, Wayne State University, Detroit, Michigan, 1996.

[23] T. K. Kroc. Low intensity configuration at NTF for microdosimetry and spectroscopy. Technical Report TM-1940, Fermi National Accelerator Laboratory, Batavia, IL., 1995.

[24] G.E. Laramore, J. M. Krall, F. J. Thomas, K. J. Russell, M. H. Maor, F. R. Hendrickson, K. L. Martz, T. W. Griffin, and L. W. Davis. Fast neutron radiotherapy for locally advanced prostate cancer. Am. J. Clin. Oncol., 16(2):164$167,1993$. 
[25] C. M. Lederer and V. S. Shirley. Table of Isotopes-Seventh Edition. Wiley Press, 1978.

[26] A. J. Lennox. Private Communication. 1997.

[27] G. L. Locher. Biological effects and therapeutic possibilities of neutrons. Am. J. Roentgenol., 36:1-13, 1936.

[28] Awschalom M., G. M. Lee, M. L: Palmer, and D. E. Young. The Fermilab neutron radiotherapy facility. IEEE Transitions on Nuclear Science, NS-24(3), 1977.

[29] R. L. Maughan, C. Kota, and M. Yudelev. A microdosimetric study of the dose enhancement in a fast neutron beam due to boron neutron capture. Phys. Med. Biol., 37:1957-1961, 1992.

[30] H. G. Menzel, P. Pihet, and A. Wambersie. Microdosimetric specification of radiation quality in neutron radiation therapy. Int. J. Radiat. Biol., 57(4):865$883,1990$.

[31] B. J. Mijnheer, J. J. Batterman, and A. Wambersie. What degree of accuracy is required and can be achieved in photon and neutron therapy. Radiother. Oncol., 8:237-252, 1987.

[32] W. Newhauser and U. J. Schrewe. Gas-to-wall absorbed dose conversion factors for neutron energies of 25 to $250 \mathrm{MeV}$. Atomic Data And Nuclear Data Tables, 65:37-53, 1997.

[33] W. D. Newhauser. Neutron kerma factor measurements in the 25-MeV to 85$\mathrm{MeV}$ neutron energy range. $\mathrm{PhD}$ thesis, University of Wisconsin, Madison, Wisconsin, 1995. 
[34] American Association of Physicists in Medicine. Protocol for Neutron Beam Dosimetry. American Institute of Physics, New York, 1980.

[35] International Commission on Radiation Units and Measurements. Physical Aspects of Radiation, volume Report 10b. International Commission on Radiation Units and Measurements, Bethesda, MD, 1964.

[36] International Commission on Radiation Units and Measurements. Radiation Quantities and Units, volume Report 33. International Commission on Radiation Units and Measurements, Bethesda, MD, 1980.

[37] International Commission on Radiation Units and Measurements. Microdosimetry, volume Report 36. International Commission on Radiation Units and Measurements, Bethesda, MD, 1983.

[38] P. Pihet. Etude microdosimetrique de faisceaux de neutrons de haute energie. Applications dosimetriques et radiobiologiques (Appendices). $\mathrm{PhD}$ thesis, Universite Catholique de Louvain, Louvain-la-Neuve, Belgium. 1989.

[39] P. Pihet, S. Gerdung, R. E. Grillmaier, A. Kunz, and H. G. Menzel. Critical assessment of calibration techniques for low pressure proportional counters used in radiation dosimetry. Radiat. Prot. Dosim., 44(1/4):115-120, 1992.

[40] P. Pihet, J. Guelette, H. G. Menzel, R. E. Grillmaier. and A. Wambersie. Use of microdosimetric data of clinical relevance in neutron therapy planning. Rad. Prot. Dos., 23(1/4):471-474, 1988.

[41] P. Pihet, H. G. Menzel, R. Schmidt, M. Beauduin, and A. Wambersie. Biological weighting function for RBE specification of neutron therapy beams. Intercomparison of 9 European centers. Rad. Prot. Dos., 31(1/4):437-442, 1990. 
[42] F. Pöller, W. Sauerwein, and J. Rassow. Monte Carlo calculation of the dose enhancement by neutron capture of B-10 in fast neutron therapy. Phys. Med. Biol., 38:397-410, 1993.

[43] I. Rosenberg and M. Awschalom. Characterization of a $p(66) B e(49)$ neutron therapy beam I: Central axis depth dose and off-axis ratios. Med. Phys., 8:99-104, 1981.

[44] I. Rosenberg, M. Awschalom, and R. Ten Haken. The effects of hydrogenous and nonhydrogenous filters on the quality of a $\mathrm{p}(66) \mathrm{Be}(49)$ neutron beam. Med. Phys., 9:199-203, 1982.

[45] M. A. Ross, P. M. DeLuca Jr., D. T. L. Jones, A. J. Lennox, and R. L. Maughan. Calculated fluence spectra at neutron therapy facilities. Radiat. Prot. Dosim., 70:481-484, 1997 .

[46] H. H. Rossi and W. Rosenweig. A device for the measurement of dose as a function of specific ionization. Radiology, 64:404, 1955.

[47] K. J. Russell, R. J. Caplan, G. E. Laramore, C. M. Burnison, M. H. Maor, M. E. Taylor, S. Zink, L. W. Davis, and T. W. Griffin. Photon versus neutron external beam radiotherapy in the treatment of locally advanced prostate cancer: Results of a randomized prospective trial. Int. J. Radiation Oncology Biol. Phys., 28:47-54, 1994.

[48] K. R. Saroja, J. Mansell, F. R. Hendrickson, L. Cohen, and A. J. Lennox. Failure of accelerated neutron therapy to control high grade astrocytomas. Int. J. Radiation Oncology Biol. Phys., 17:1295-1297, 1989.

[49] J. J. Schrewe, H. J. Brede, and G. Dietz. Dosimetry in mixed neutronphoton fields with tissue-equivalent proportional counters. Rad. Prot. Dos., 29(1/4):41-45, 1989. 
[50] U. J. Schrewe, W. G. Alberts, W. D. Newhauser, H. J. Brede, and P. M. DeLuca Jr. Comparison of various dose quantities in tissue and tissue substitutes at neutron energies between $20 \mathrm{MeV}$ and $100 \mathrm{MeV}$. Rad. Prot. Dosim., 70:17-20, 1997.

[51] U. J. Schrewe, H. J. Brede, S. Gerdung, R. Nolte, P. Pihet, P. Schmelzbach, and $\mathrm{H}$. Schumacher. Determination of kerma factors of A-150 plastic and carbon at neutron energies between 45 and $66 \mathrm{MeV}$. Rad. Prot. Dosim., pages 21-24, 1992.

[52] J. P. Slabbert, P. J. Binns, H. L. Jones, and J. H. Hough. A quality assessment of the effects of a hydrogenous filter on a $\mathrm{p}(66) \mathrm{Be}(40)$ neutron beam. Brit. $J$. Radiol., 62:989-994, 1989.

[53] J. B. Smathers, V. A. Otte, A. R. Smith, P. R. Almond, F. H. Attix, J. J. Spokas, W. M. Quam, and L. J. Goodman. Composition of A-150 tissue equivalent plastic. Med. Phys., 4:74, 1975.

[54] R. S. Stone. Neutron therapy and specific ionization.'Am. J. Roentgenol., 59:771-785, 1948.

[55] R. S. Stone, J. H. Lawrence, and P. C. Aebersold. A preliminary report on the use of fast neutrons in the treatment of malignant disease. Radiology, 35:322-327, 1940 .

[56] L. M. Van Putten and R. F. Kallman. Oxygenation status of a transplantable tumor during fractionated radiation therapy. J. Natl. Cancer Inst., 40:441$451,1968$.

[57] A.J. Waker. Experimental uncertainties in microdosimetric measurements and an examination of the performance of three commercially produced proportional counters. Nucl. Instr. and Meth., A234:354-360, 1985. 
[58] A. Wambersie. Neutron therapy: From radiobiological expectation to clinical reality. Radiat. Prot. Dosim., 44:379-395, 1992.

[59] A. Wambersie. Contribution of microdosimetry to the specification of neutron beam quality for the choice of the 'clinical RBE' in fast neutron therapy. Radiat. Prot. Dosim., 52:453-460, 1994.

[60] A. Wambersie and H. G. Menzel. Specification of absorbed dose and radiation quality in heavy particle therapy (A review). Radiat. Prot. Dosim., 70:517$527,1997$.

[61] F. M. Waterman, F. T. Kuchnir, L. S. Skaggs, D. K. Bewley, B. C. Page, and F. H. Attix. The use of B-10 to enhance the tumor dose in fast neutron therapy. Phys. Med. Biol., 23:592-602, 1978.

[62] P. Wootton, R. Risler, J. Livesey, S. Brossard, G. Laramore, and T. Griffin. Boron neutron capture enhancement of the tumor dose in fast neutron therapy beams. In Progress in Neutron Therapy for Cancer, New York, United States of America, 1992. Plenum Press.

[63] C. S. Wuu, H. I. Amols, P. Kilauga, L. E. Reinstein, and S. Saraf. Microdosimetry for boron neutron capture therapy. Radiat. Res., 130:355-359, 1992.

[64] P. G. Young, E. D. Arthur, M. Bozoain, T. R. England, G. M. Hall, R. J. LaBauve, R. C. Little, R. E. MacFarlane, D. G. Madland, R. T. Perry, and W. B. Wilson. Transport libraries for incident proton and neutron energies to $100 \mathrm{MeV}$. Technical Report LA-11753-MS, Los Alamos National Laboratory, Los Alamos, NM, USA, 1990. 